\title{
Boosting Metathesis Activity of Molybdenum Oxo Alkylidenes by Tuning the Anionic Ligand $\sigma$-Donation
}

Jordan De Jesus Silva,,$^{\S} a$ Margherita Pucino, ${ }^{\S, a}$ Feng Zhai, ${ }^{b}$ Deni Mance, ${ }^{a}$ Zachariah J. Berkson, ${ }^{a}$ Darryl F. Nater, ${ }^{a}$ Amir H. Hoveyda, ${ }^{c}$ Christophe Copéret, ${ }^{*, a}$ and Richard R. Schrock*,b

${ }^{a}$ Department of Chemistry and Applied Biosciences, ETH Zürich, Vladimir-Prelog-Weg 1-5, CH-8093 Zürich, Switzerland

${ }^{b}$ Department of Chemistry, Massachusetts Institute of Technology, Cambridge, Massachusetts, 02139, United States

${ }^{c}$ Department of Chemistry, Merkert Chemistry Center, Boston College, Chestnut Hill, Massachusetts 02467, United States

* Corresponding authors

$\S$ These authors contributed equally to this work.

ccoperet@ethz.ch

rrs@mit.edu 


\section{Table of Contents}

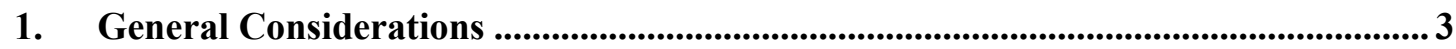

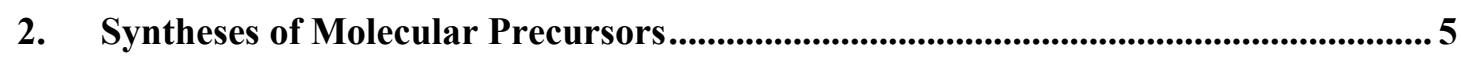

3. Synthesis of Silica-Supported Complexes ............................................................ 10

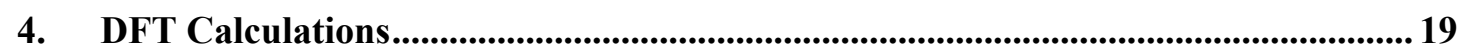

5. Experimental Procedures and Catalytic Data .........................................................38

Plots of Conversion vs Time and Selectivity vs Time for Cis-4-Nonene Experiments..... 40

Plots of Conversion vs Time and Selectivity vs Time for 1-Nonene Experiments ............ 60

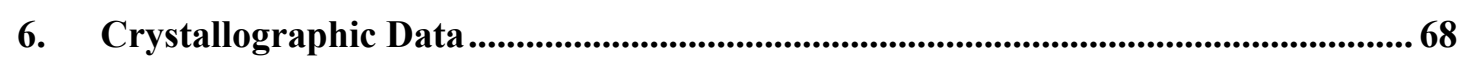

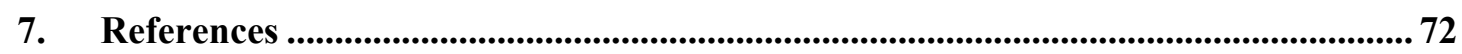




\section{General Considerations}

All experiments were carried out under an inert nitrogen or argon atmosphere using Schlenk techniques or an MBraun or GS glovebox equipped with a purifier unit. Water and oxygen levels were kept below $0.1 \mathrm{ppm}$. Toluene, $\mathrm{CH}_{2} \mathrm{Cl}_{2}$ and pentane were purified using double MBraun SPS alumina columns. Benzene and benzene- $d_{6}$ were distilled from $\mathrm{Na}$ /benzophenone. All solvents were degassed by three consecutive freeze-pump-thaw cycles.

${ }^{13} \mathrm{C}$-labeled 4-vinylanisole was purchased from Organochem KFT, Hungary and used without further purification (purity $>95 \%$, packed and stored under inert atmosphere at $-35^{\circ} \mathrm{C}$ ). Lowtemperature diffraction data were collected on a Bruker-ApexII diffractometer. Elemental analyses were performed at Mikroanalytisches Labor Pascher, Germany. All infrared (IR) spectra were recorded using a Bruker FT-IR Alpha spectrometer placed inside a glovebox, equipped with the OPUS software. The IR spectrometer had a total spectral range of 275-7500 $\mathrm{cm}^{-1}$ with a resolution $<2 \mathrm{~cm}^{-1}$ and consisted of a RockSolid interferometer, a DTGS (triglycine sulfate) detector and a $\mathrm{SiC}$ globar source. Solid samples were investigated in a magnetic pellet holder. A typical experiment consisted of 32 consecutive transmission measurements in the region from 4000 to $400 \mathrm{~cm}^{-1}$. Solution NMR spectra were obtained on Bruker Avance 400 $\mathrm{MHz}$ and Bruker Neo $500 \mathrm{MHz}$ spectrometers at ambient temperature under arbitrary concentration. ${ }^{1} \mathrm{H}$ and ${ }^{13} \mathrm{C}$ chemical shifts are referenced relative to the residual solvent peak and reported relative to tetramethylsilane $(\delta=0 \mathrm{ppm}) \cdot{ }^{1} \mathrm{H}$ and ${ }^{13} \mathrm{C}$ solid-state NMR experiments were acquired on a Bruker AVANCE III spectrometer operating at $400 \mathrm{MHz}$ ${ }^{1} \mathrm{H}$ frequency (9.4 T) and equipped with a $3.2 \mathrm{~mm}{ }^{1} \mathrm{H}$, X MAS probe (Bruker). ${ }^{19} \mathrm{~F}$ solid-state NMR experiments were acquired on a Bruker AVANCE III spectrometer operating at $700 \mathrm{MHz}{ }^{1} \mathrm{H}$ frequency $(16.4 \mathrm{~T})$. The MAS frequency was set to 10 or $16 \mathrm{kHz}$. For the ${ }^{13} \mathrm{C}\left\{{ }^{1} \mathrm{H}\right\}$ CP-MAS measurements, Hartman-Hahn cross-polarization was performed by making use of a linear ramp from 70 to $100 \% .100 \mathrm{kHz}{ }^{1} \mathrm{H}$ SPINAL64 ${ }^{[1]}$ heteronuclear decoupling was applied during the ${ }^{13} \mathrm{C}$ acquisition period. ${ }^{13} \mathrm{C}$ solid-state NMR spectra are referenced externally with respect to the methylene signal of adamantane $(38.48 \mathrm{ppm}) .{ }^{1} \mathrm{H}$ solid-state NMR spectra were acquired using a $\pi / 2-\tau-\pi / 2-\tau$ echo sequence with an echo delay $\tau$ of $0.625 \mathrm{~ms}$ (10 rotor periods). The ${ }^{1} \mathrm{H}$ and ${ }^{13} \mathrm{C}$ MAS NMR spectra of $\mathbf{1}^{*} @ \mathrm{SiO}_{2}$ were acquired at a reduced temperature using a variable temperature chiller unit to maintain the temperature in the MAS gas stream at $270 \mathrm{~K}$ in order to mitigate decomposition of the supported compound. All other MAS NMR spectra were acquired at ambient temperature. 
Liquid catalytic test aliquots were analyzed using a GC/FID (Agilent Technologies 7890 A) equipped with a split-splitless injector heated to $250{ }^{\circ} \mathrm{C}$, injection volume $0.5 \mu \mathrm{L}$ using hydrogen carrier gas. Chromatographic separations for 1-nonene and cis-4-nonene catalytic tests were performed using an HP-5 (Agilent Technologies) column (30 m, 0.32 mm, $0.25 \mu \mathrm{m}$ stationary phase).

Substrates and internal standard. 1 -Nonene (TCI $\geq 95 \%$ ), cis-4-nonene (TCI $\geq 95 \%$ ), decaline (Sigma Aldrich 98\%) and toluene were distilled from $\mathrm{Na}$, degassed by three consecutive freeze-pump-thaw cycles, filtered through activated alumina, and stored for 4 hours over activated Selexsorb $\mathrm{CD}^{\circledR}$ and activated $4 \AA$ molecular sieves.

Silica. Silica (Aerosil Degussa, $200 \mathrm{~m}^{2} \mathrm{~g}^{-1}$ ) was compacted with distilled water, sieved, calcined at $500{ }^{\circ} \mathrm{C}$ under air for $12 \mathrm{~h}$ and treated under vacuum $\left(10^{-5} \mathrm{mbar}\right)$ at $500{ }^{\circ} \mathrm{C}$ for $8 \mathrm{~h}$ and then at $700{ }^{\circ} \mathrm{C}$ for $14 \mathrm{~h}$ (referred to as $\mathrm{SiO}_{2-700)}$. ${ }^{[2]}$ 


\section{Syntheses of Molecular Precursors}

Molecular precursors $\left[\mathrm{Mo}(\mathrm{O})\left\{\mathrm{CH}-4-(\mathrm{MeO}) \mathrm{C}_{6} \mathrm{H}_{4}\right\}(\mathrm{THF})_{2}\left\{\mathrm{OC}\left(\mathrm{CF}_{3}\right)_{3}\right\}_{2}\right](\mathbf{1})$ and $[\mathrm{Mo}(\mathrm{O})\{\mathrm{CH}-$ 4-(MeO) $\left.\mathrm{C}_{6} \mathrm{H}_{4}\right\}(\mathrm{OTPP})_{2}$ ] (2) were synthesized according to literature procedures. ${ }^{[3]}$ The corresponding NMR spectra are added for comparison to the grafted analogs.

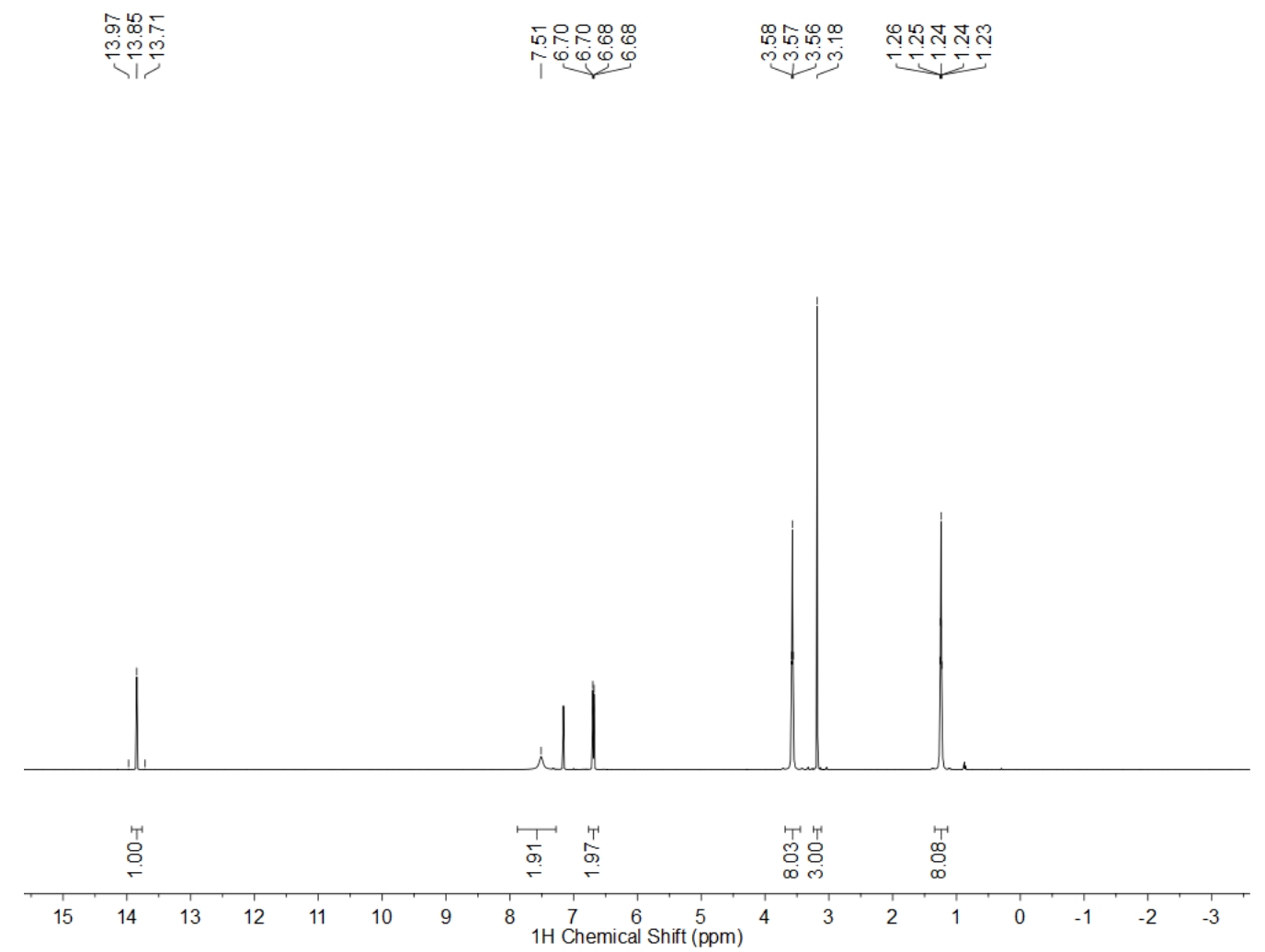

Figure S1. ${ }^{1} \mathrm{H}$ NMR $\left(500 \mathrm{MHz}, \mathrm{C}_{6} \mathrm{D}_{6}\right)$ spectrum of $\mathbf{1}$. 


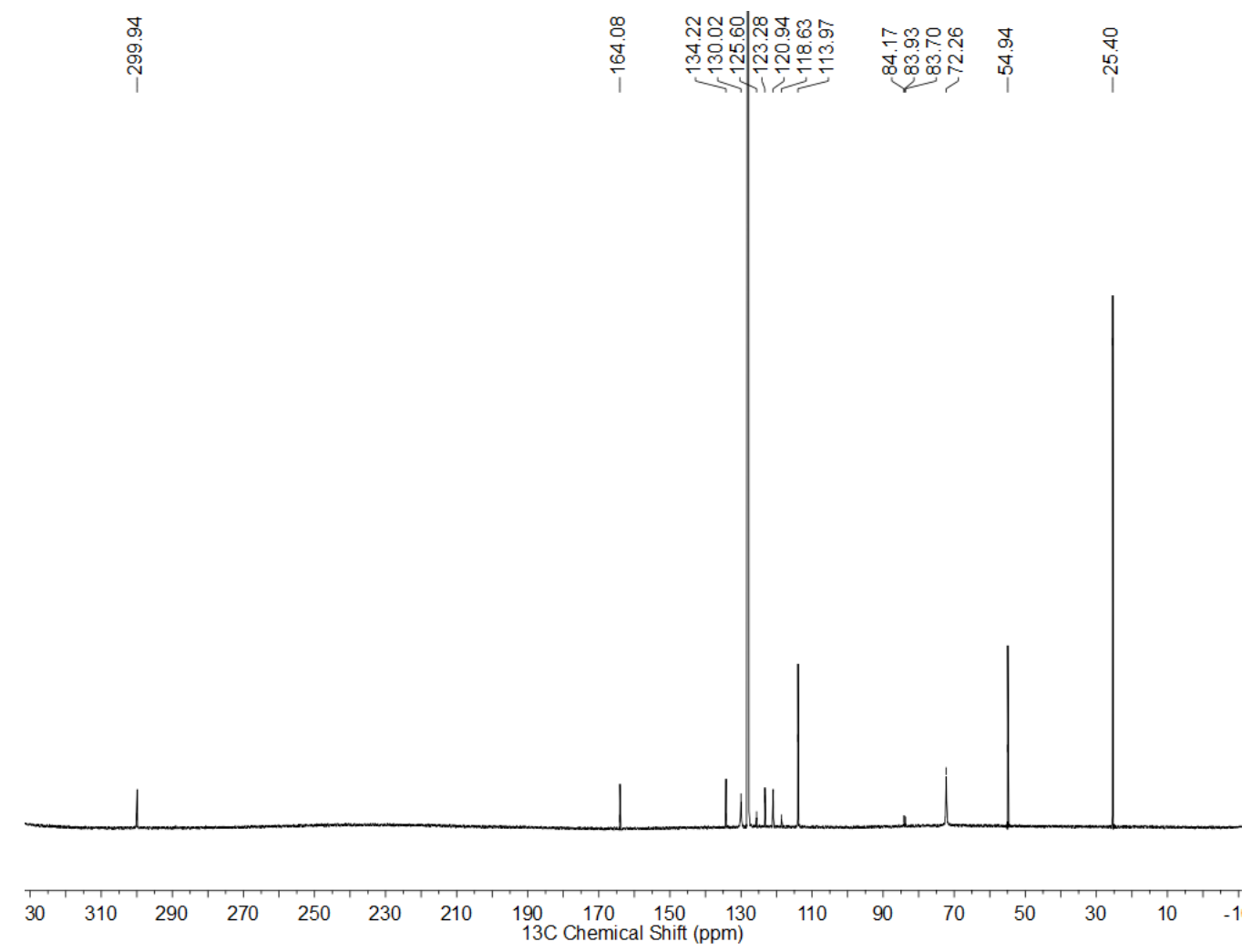

Figure S2. ${ }^{13} \mathrm{C}\left\{{ }^{1} \mathrm{H}\right\}$ NMR $\left(126 \mathrm{MHz}, \mathrm{C}_{6} \mathrm{D}_{6}\right)$ spectrum of $\mathbf{1}$.

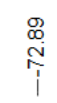

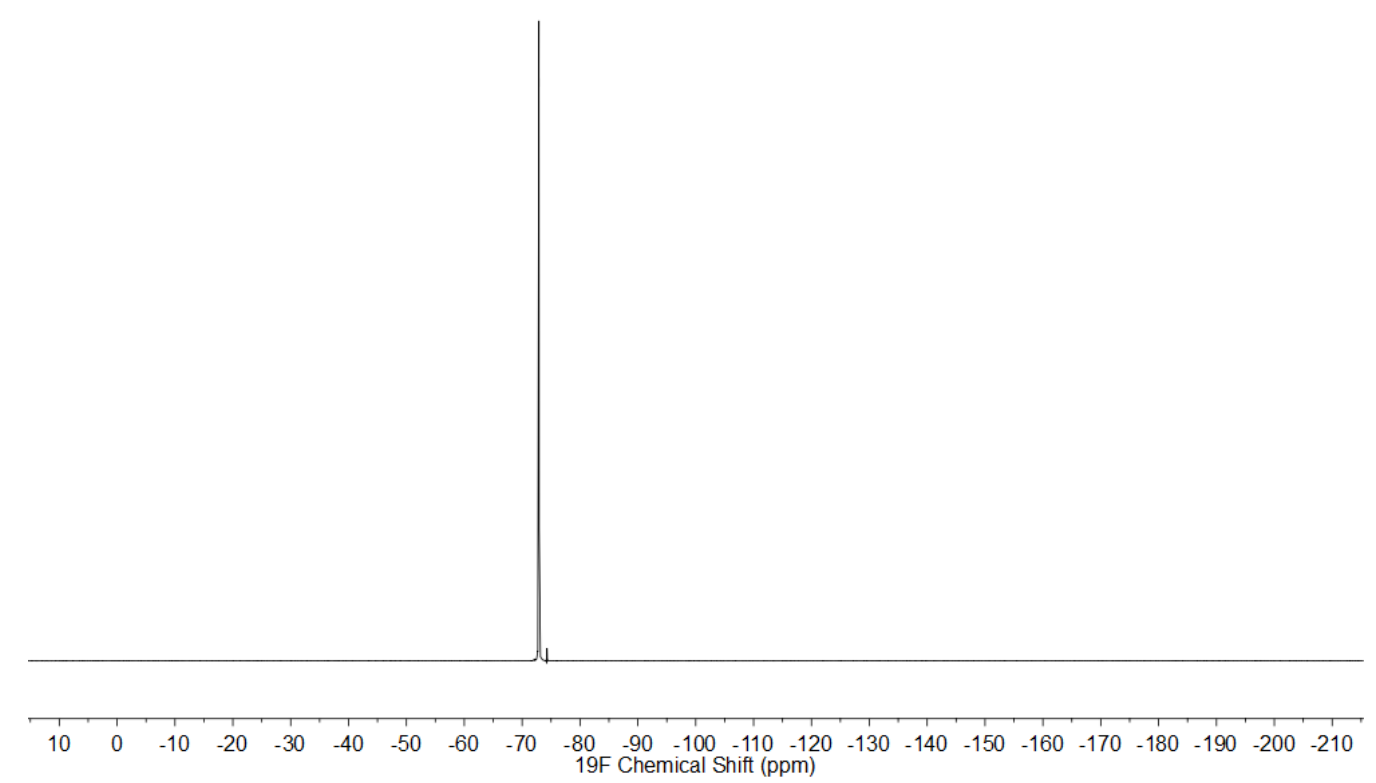

Figure S3. ${ }^{19} \mathrm{~F}$ NMR $\left(470 \mathrm{MHz}, \mathrm{C}_{6} \mathrm{D}_{6}\right)$ spectrum of $\mathbf{1}$. 


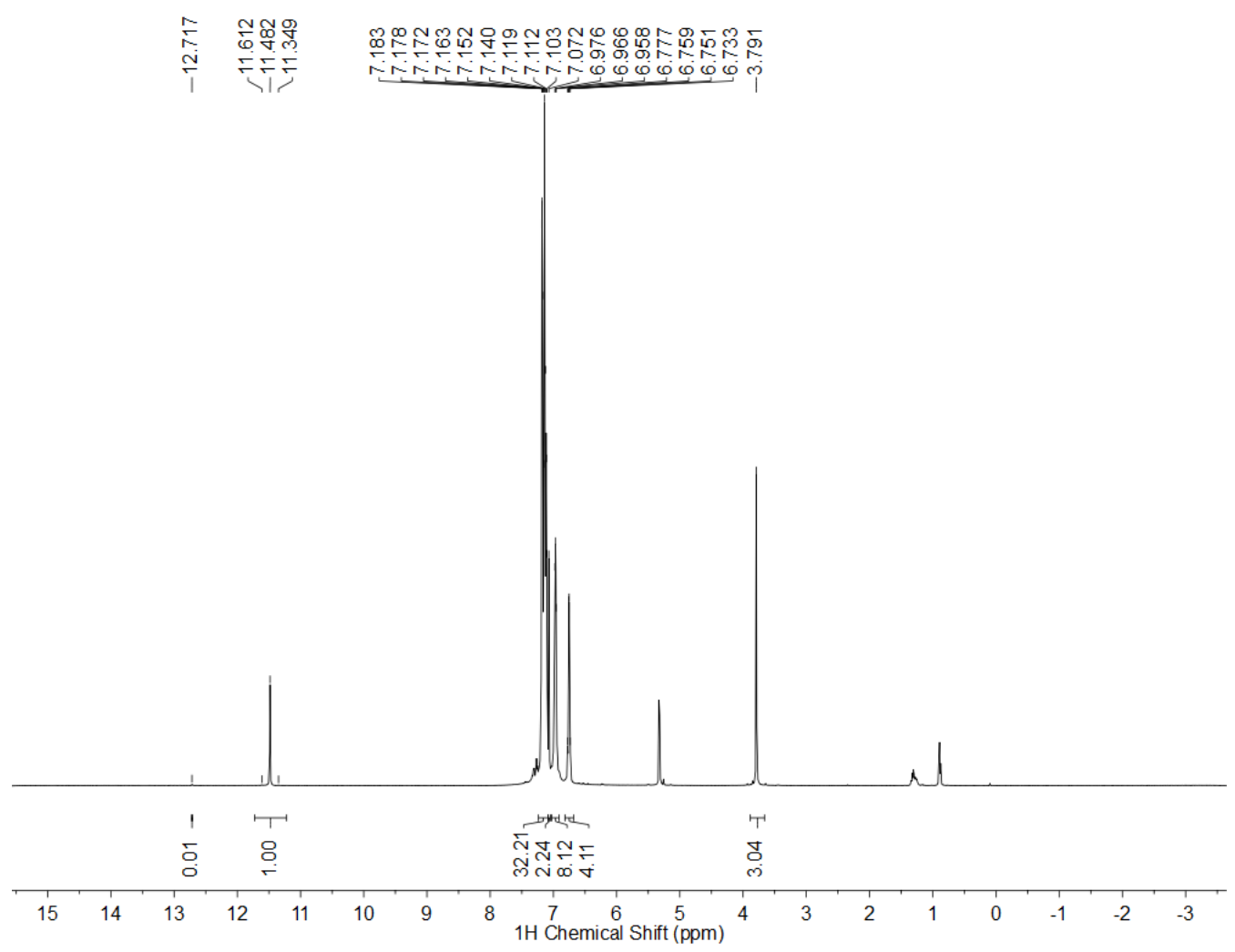

Figure S4. ${ }^{1} \mathrm{H} \mathrm{NMR}\left(500 \mathrm{MHz}, \mathrm{CD}_{2} \mathrm{Cl}_{2}\right)$ spectrum of 2.

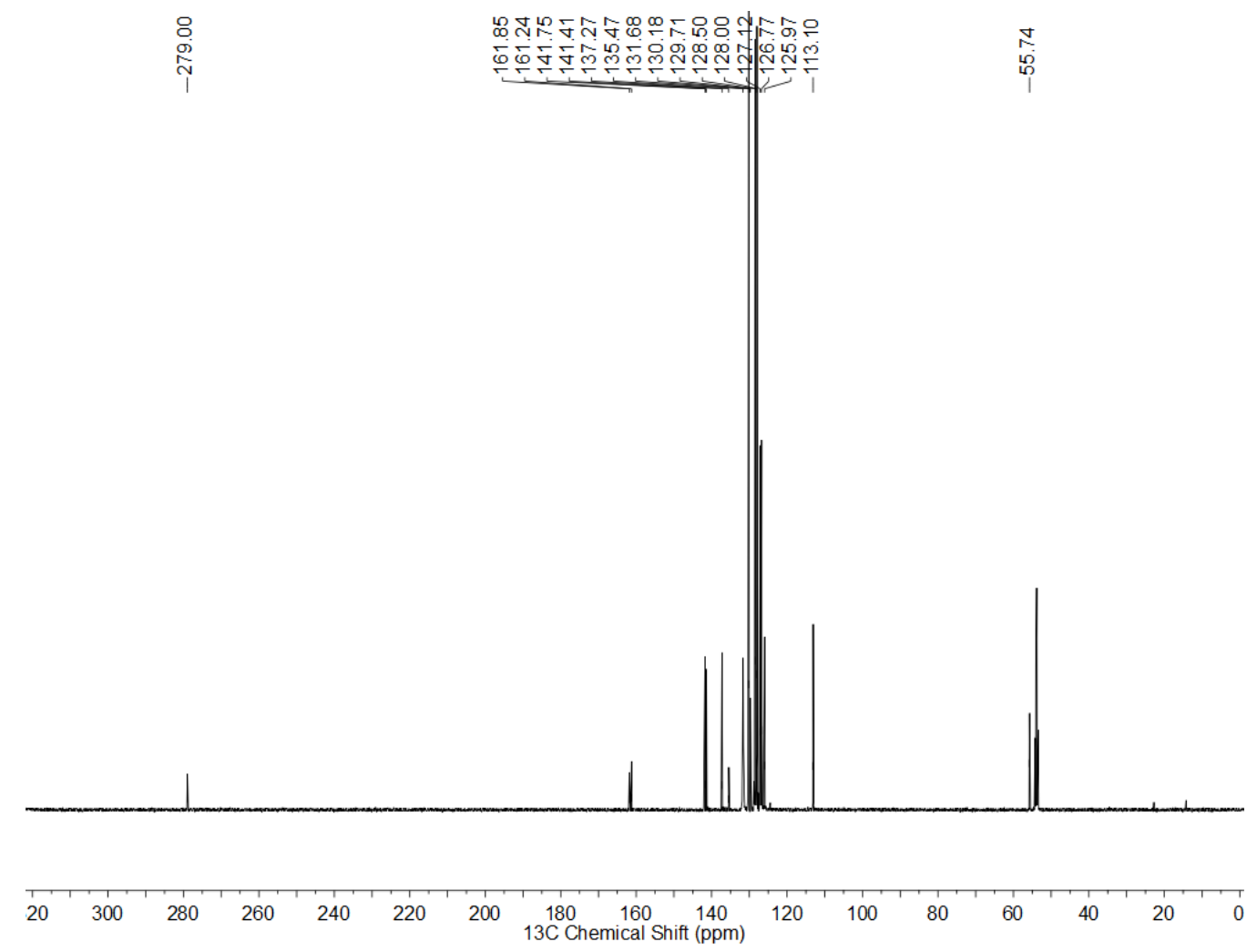

Figure S5. ${ }^{13} \mathrm{C}\left\{{ }^{1} \mathrm{H}\right\} \mathrm{NMR}\left(126 \mathrm{MHz}, \mathrm{CD}_{2} \mathrm{Cl}_{2}\right)$ spectrum of 2 . 


\section{$\left[\mathrm{Mo}(\mathrm{O})\left\{\mathrm{C} * \mathrm{H}-4-(\mathrm{MeO}) \mathrm{C}_{6} \mathrm{H}_{4}\right\}(\mathrm{THF})\left\{\mathrm{OC}\left(\mathrm{CF}_{3}\right)_{3}\right\}_{2}\right], 1^{*}$}

A red solution of 1 (25 mg, $0.029 \mathrm{mmol}, 1$ equiv.) in $\mathrm{C}_{6} \mathrm{D}_{6}\left(2 \mathrm{~mL}, 6^{\circ} \mathrm{C}\right)$ was added to a solution of ${ }^{13} \mathrm{C}$-labeled 4-vinylanisole (20 mg, $0.148 \mathrm{mmol}, 5.1$ equiv.) in $\mathrm{C}_{6} \mathrm{D}_{6}(0.5 \mathrm{~mL})$ at $25{ }^{\circ} \mathrm{C}$ in an Argon filled glovebox. The solution was stirred over 1 hour at room temperature. The resulting red solution was dried thoroughly under vacuum at room temperature, the red solid was then recrystallized from $n$-pentane $(5 \mathrm{~mL})$ at $-40{ }^{\circ} \mathrm{C}$ to afford $16 \mathrm{mg}$ of the title compound (yield $71 \%$ with $63 \%$ labeling). The compound is obtained as a mixture of syn/anti isomer in ca. $4 / 1$ ratio, and the structure of the major isomer was confirmed by X-ray crystallography (vide infra). NMR data for the major (syn) isomer are listed as follows: ${ }^{1} \mathrm{H}$ NMR $\left(500 \mathrm{MHz}, \mathrm{C}_{6} \mathrm{D}_{6}\right): \delta=$ $13.66\left(\mathrm{~s},{ }^{1} J_{\mathrm{CH}}=130 \mathrm{~Hz}, 1 \mathrm{H}, \mathrm{CHAr}\right.$ ) $, 7.42\left(\mathrm{br}, 2 \mathrm{H}, o-\mathrm{Ar}_{\mathrm{p}}\right), 6.66-6.64\left(\mathrm{~m}, 2 \mathrm{H}, m-\mathrm{Ar}_{\mathrm{p}}\right.$ ), $3.42(\mathrm{br}$, 4H, THF), 3.15 (s, 3H, OCH $), 1.00-0.97$ (m, 4H, THF). ${ }^{13} \mathrm{C}\left\{{ }^{1} \mathrm{H}\right\} \mathrm{NMR}\left(126 \mathrm{MHz}, \mathrm{C}_{6} \mathrm{D}_{6}\right): \delta=$ $297.4\left(C \mathrm{HAr}_{\mathrm{p}}\right), 163.7\left(p-\mathrm{Ar}_{\mathrm{p}}\right), 133.5($ ipso-Ar $), 126.4\left(o-\mathrm{Ar}_{\mathrm{p}}\right), 121.6\left(\mathrm{q},{ }^{1} J_{\mathrm{FC}}=294, C \mathrm{~F}_{3}\right), 113.6$ $\left(m-\mathrm{Ar}_{\mathrm{p}}\right), 75.9$ (br, THF), $54.6\left(\mathrm{OCH}_{3}\right), 24.8$ (THF). The $C\left(\mathrm{CF}_{3}\right)_{3}$ carbon resonances were not observed. Only the discernable NMR features of the minor (anti) isomer are listed as follows: ${ }^{1} \mathrm{H}$ NMR $\left(500 \mathrm{MHz}, \mathrm{C}_{6} \mathrm{D}_{6}\right): \delta=14.12\left(\mathrm{~s},{ }^{1} J_{\mathrm{CH}}=146 \mathrm{~Hz}, 1 \mathrm{H}, \mathrm{CHAr}\right), 6.82-6.77(\mathrm{~m}, 2 \mathrm{H}$, anti$\left.m-\mathrm{Ar}_{\mathrm{p}}\right) \cdot{ }^{13} \mathrm{C}\left\{{ }^{1} \mathrm{H}\right\}$ NMR $\left(126 \mathrm{MHz}, \mathrm{C}_{6} \mathrm{D}_{6}\right): \delta=300.1\left(C \mathrm{HAr}_{\mathrm{p}}\right), 133.1$ (ipso-Ar $\left.\mathrm{p}\right), 128.5\left(o-\mathrm{Ar}_{\mathrm{p}}\right)$.

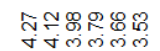

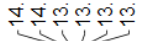

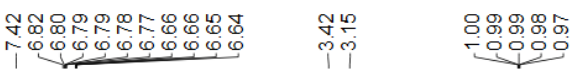

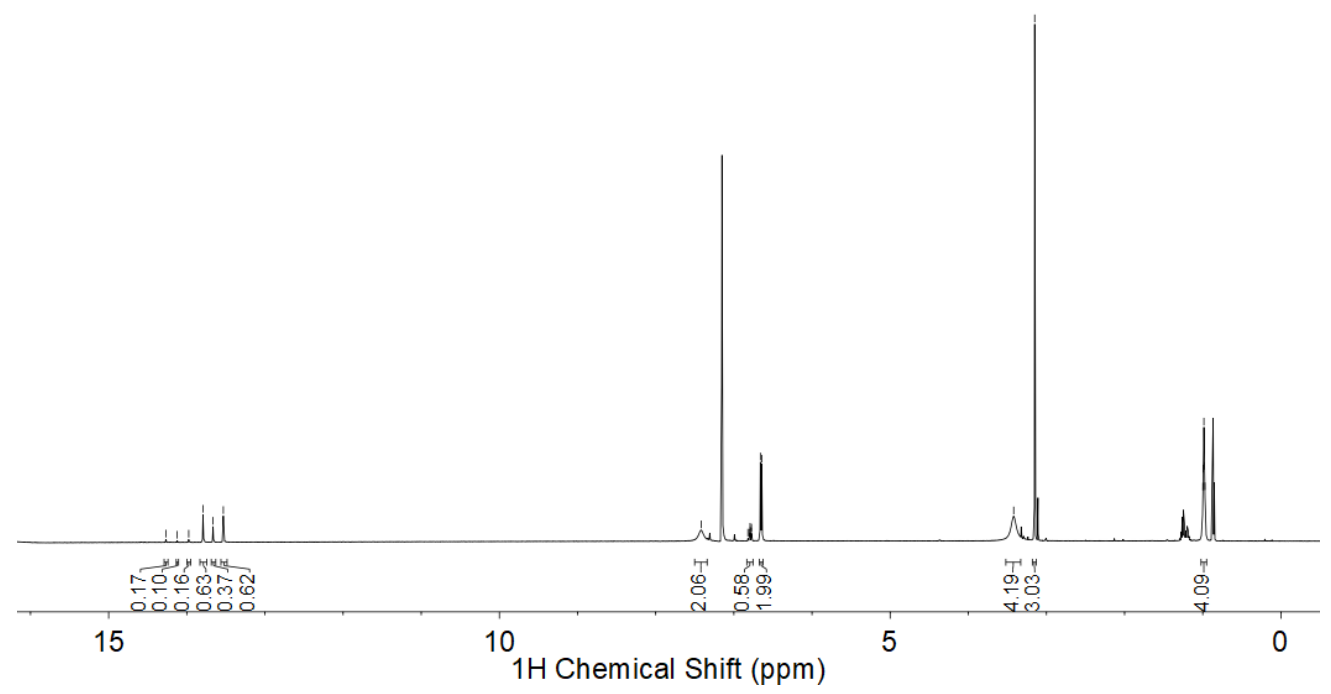

Figure S6. ${ }^{1} \mathrm{H}$ NMR $\left(500 \mathrm{MHz}, \mathrm{C}_{6} \mathrm{D}_{6}\right)$ spectrum of partially carbon-13 labeled 1* on the alkylidene carbon with trace amount of residual pentane. 


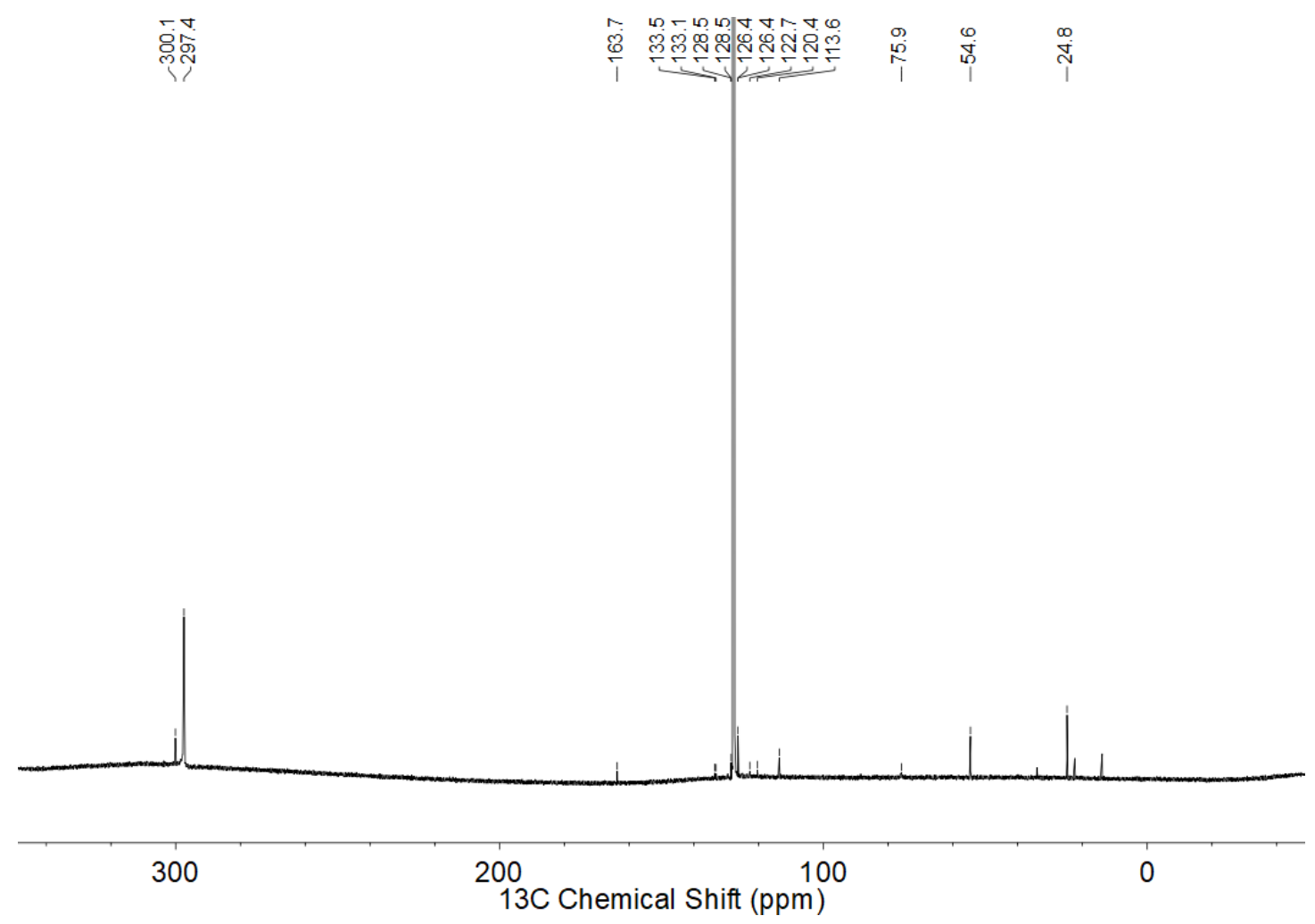

Figure S7. ${ }^{13} \mathrm{C}\left\{{ }^{1} \mathrm{H}\right\}$ NMR $\left(126 \mathrm{MHz}, \mathrm{C}_{6} \mathrm{D}_{6}\right)$ spectrum of $\mathbf{1}^{*}$. 


\title{
3. Synthesis of Silica-Supported Complexes
}

\author{
$\left[(\equiv \mathrm{SiO}) \mathrm{Mo}(\mathrm{O})\left\{\mathrm{CH}-4-(\mathrm{MeO}) \mathrm{C}_{6} \mathrm{H}_{4}\right\}(\mathrm{THF})_{x}\left\{\mathrm{OC}\left(\mathrm{CF}_{3}\right)_{3}\right\}\right], \mathbf{1} @ \mathrm{SiO}_{2}$
}

A red solution of 1 (33.2 mg, $0.0392 \mathrm{mmol}, 1$ equiv.) was added to a suspension of $\mathrm{SiO}_{2-700}$ (138.5 mg, $0.0395 \mathrm{mmol} \mathrm{SiOH}, 1$ equiv.) in cold toluene $\left(3 \mathrm{~mL},-30{ }^{\circ} \mathrm{C}\right)$ at $25^{\circ} \mathrm{C}$ in an Argon filled glovebox. The suspension was slowly stirred (120 rpm) over $3 \mathrm{~h}$. The solid was collected by filtration and washed by suspension/filtration cycles in toluene $(3 \times 1 \mathrm{~mL})$, benzene $(3 \mathrm{x}$ $1 \mathrm{~mL})$ and pentane $(3 \times 2 \mathrm{~mL})$. The resulting brown solid was dried thoroughly under high vacuum $\left(10^{-5} \mathrm{mbar}\right)$ at room temperature for 2 hours to afford $162 \mathrm{mg}$ of the titled compound. All filtrate solutions were collected and analyzed by ${ }^{19} \mathrm{~F}$ NMR spectroscopy in $\mathrm{C}_{6} \mathrm{D}_{6}$ using 4,4'-difluorobiphenyl as internal standard, indicating that $0.039 \mathrm{mmol}$ of $\mathrm{HOC}\left(\mathrm{CF}_{3}\right)_{3}$ were released, corresponding to quantitative grafting. Mo 2.27\%, C $3.80 \%, \mathrm{H} 0.38 \%$, F 2.39\%, corresponding to $13.4 \mathrm{C} / \mathrm{Mo}$ (12-16 expected), $15.9 \mathrm{H} / \mathrm{Mo}$ (8-16 expected), $5.3 \mathrm{~F} / \mathrm{Mo}$ (9 expected).

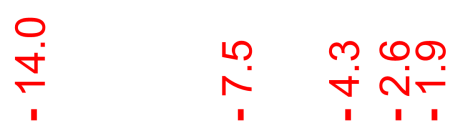

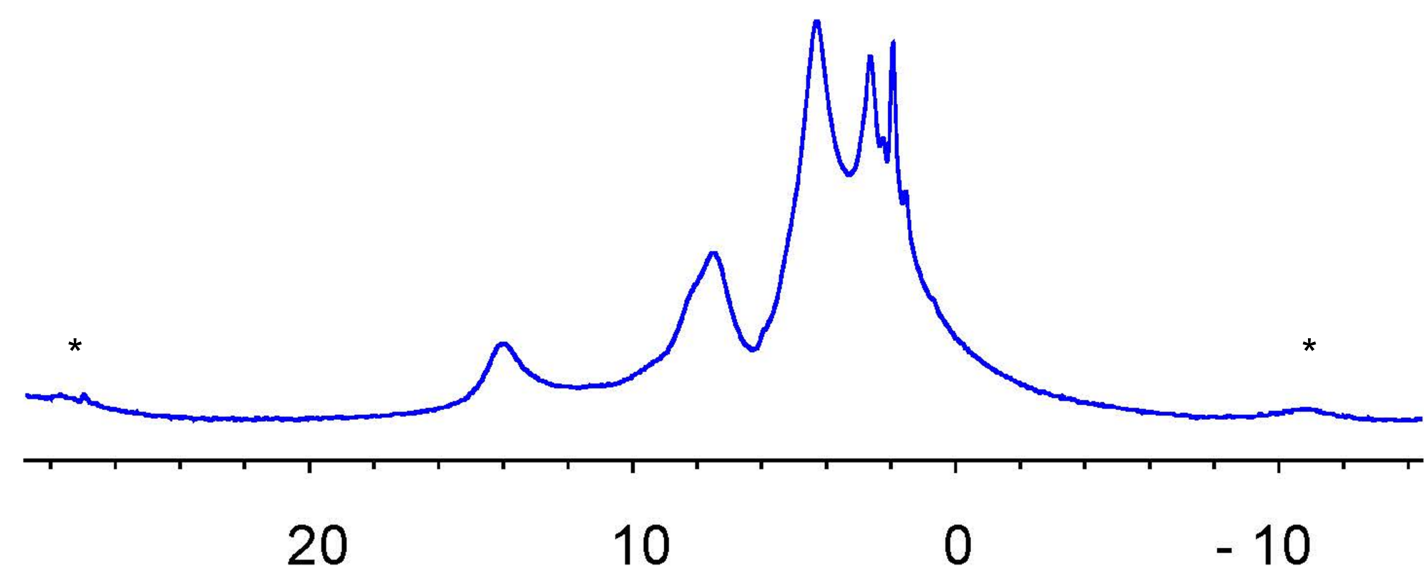

\section{${ }^{1} \mathrm{H}$ Chemical Shift (ppm)}

Figure S8. ${ }^{1} \mathrm{H}$ MAS NMR spectrum of $1 @ \mathrm{SiO}_{2}$, acquired at 9.4 T, 298 K, 10 kHz MAS, with a $2 \mathrm{~s}$ recycle delay. (* spinning side bands) 


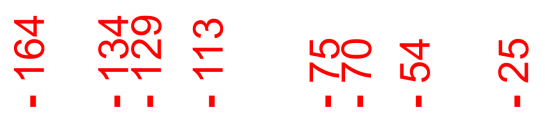

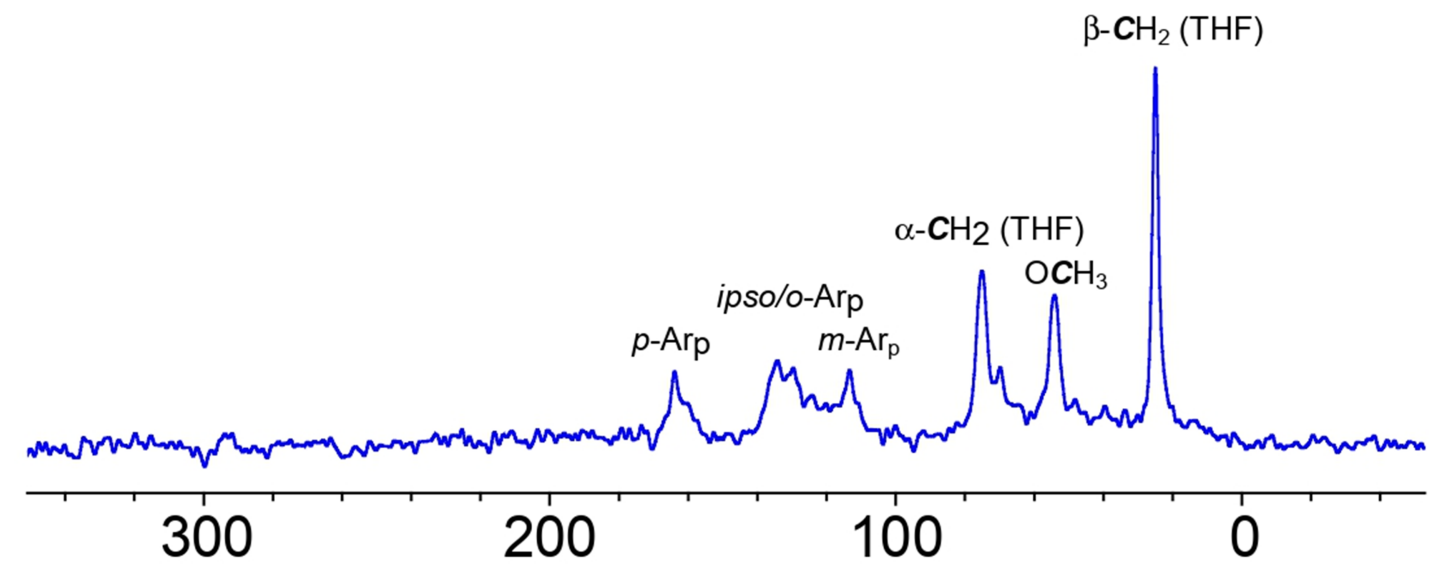

${ }^{13} \mathrm{C}$ Chemical Shift (ppm)

Figure S9. ${ }^{13} \mathrm{C}$ CP MAS NMR spectrum of $1 @ \mathrm{SiO}_{2}$, acquired at $9.4 \mathrm{~T}, 298 \mathrm{~K}, 10 \mathrm{kHz} \mathrm{MAS}$, with a $2 \mathrm{~s}$ recycle delay and with a ${ }^{13} \mathrm{C}-{ }^{1} \mathrm{H}$ contact time of $3 \mathrm{~ms}$. 


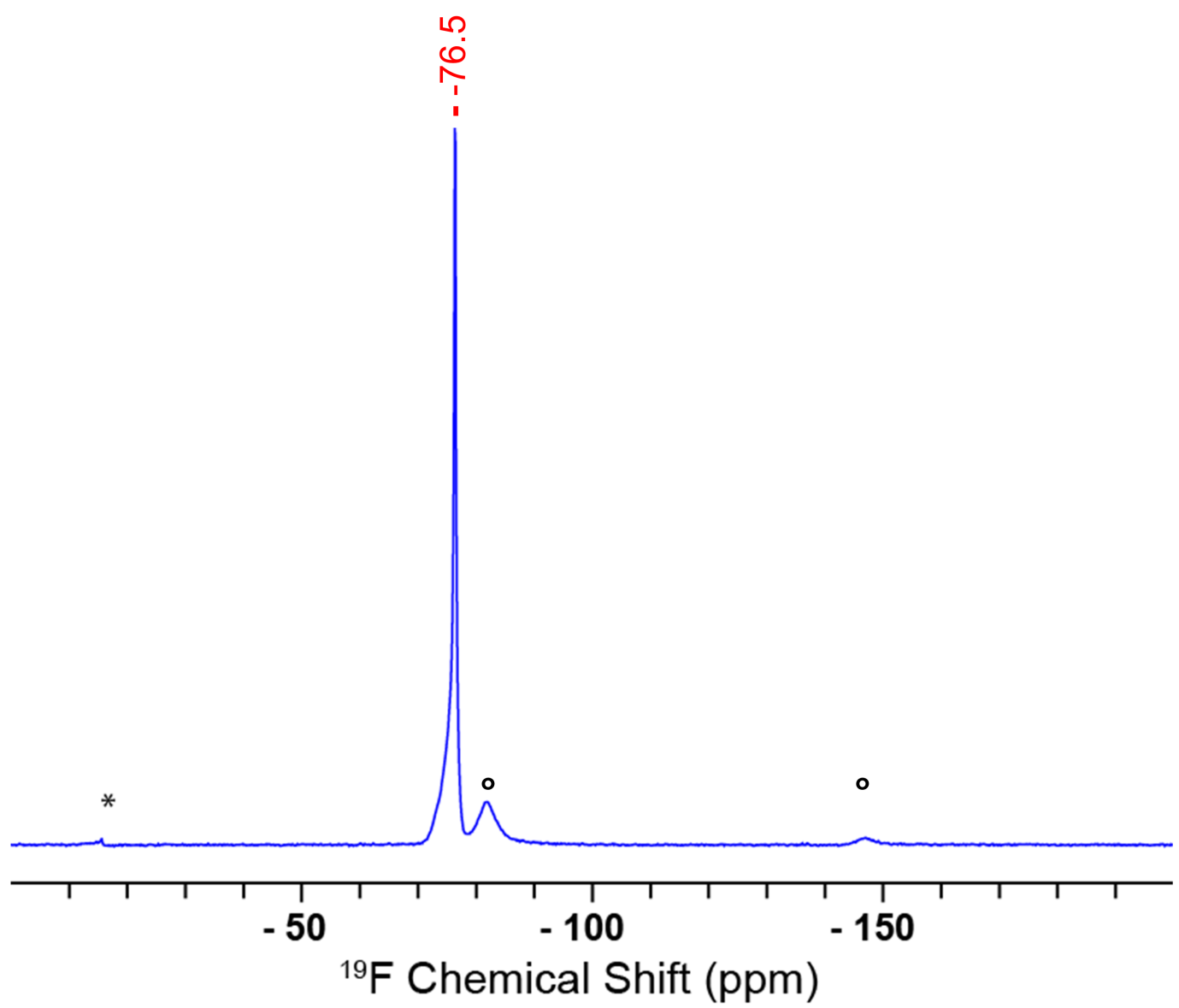

Figure S10. ${ }^{19} \mathrm{~F}$ MAS NMR spectrum of $1 @ \mathrm{SiO}_{2}$, acquired at $16.4 \mathrm{~T}, 298 \mathrm{~K}, 10 \mathrm{kHz}$ MAS, with a 2 s recycle delay. (* spinning side bands, ${ }^{\circ}$ probe/rotor background). 


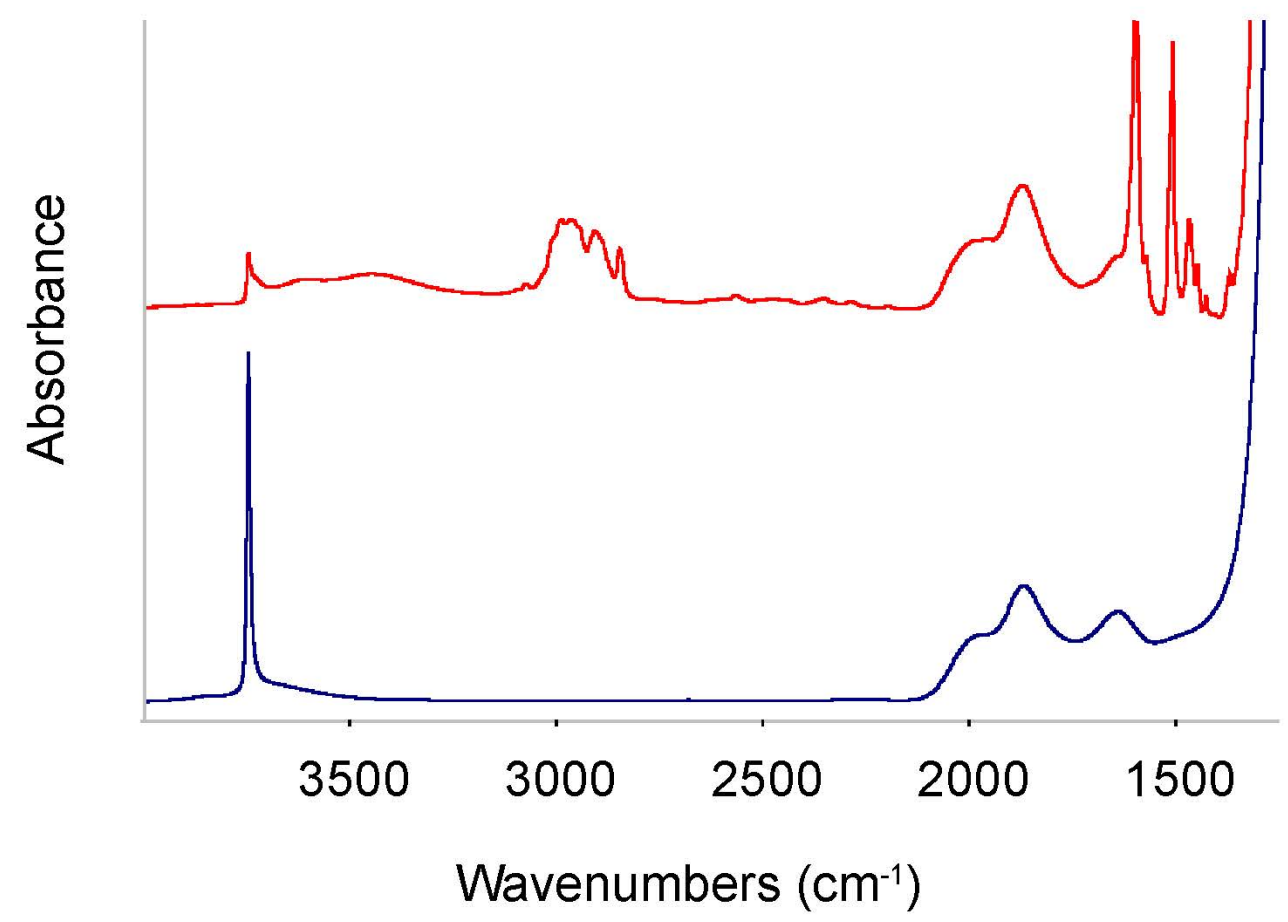

Figure S11. Transmission FT-IR spectra (from top) of $1 @ \mathrm{SiO}_{2}$ red, and $\mathrm{SiO}_{2-(700)}$ blue.

\section{[(=SiO)Mo(O) $\left\{\mathbf{C H}-4-(\mathrm{MeO}) \mathbf{C}_{6} \mathbf{H}_{4}\right\}$ (TPPO)], $2 @ \mathrm{SiO}_{2}$}

A red solution of 2 (12.6 mg, $0.0123 \mathrm{mmol}, 1.07$ equiv.) was added to a suspension of $\mathrm{SiO}_{2-700}$ (40.4 mg, $0.0115 \mathrm{mmol} \mathrm{SiOH,} 1$ equiv.) in toluene ( $3 \mathrm{~mL}$ ) at $25^{\circ} \mathrm{C}$ in an Argon filled glovebox. The suspension was slowly stirred $(120 \mathrm{rpm})$ over $3 \mathrm{~h}$. The solid was collected by filtration and washed by suspension/filtration cycles in toluene $(3 \times 1 \mathrm{~mL})$ and benzene $(3 \times 1 \mathrm{~mL})$ and pentane $(3 \times 2 \mathrm{~mL})$. The resulting orange solid was dried thoroughly under high vacuum $\left(10^{-5} \mathrm{mbar}\right)$ at room temperature for 2 hours to afford $45 \mathrm{mg}$ of the title compound. All filtrate solutions were collected and analyzed by ${ }^{1} \mathrm{H}$ NMR spectroscopy in $\mathrm{C}_{6} \mathrm{D}_{6}$ using ferrocene as internal standard, indicating that $0.0069 \mathrm{mmol}$ of HOTPP were released in solution, corresponding to $60 \%$ grafting. Mo $1.65 \%$, C $9.27 \%$, $\mathrm{H} 0.56 \%$, corresponding to $44.9 \mathrm{C} / \mathrm{Mo}$ (38 expected), $32.2 \mathrm{H} / \mathrm{Mo}$ (29 expected). 


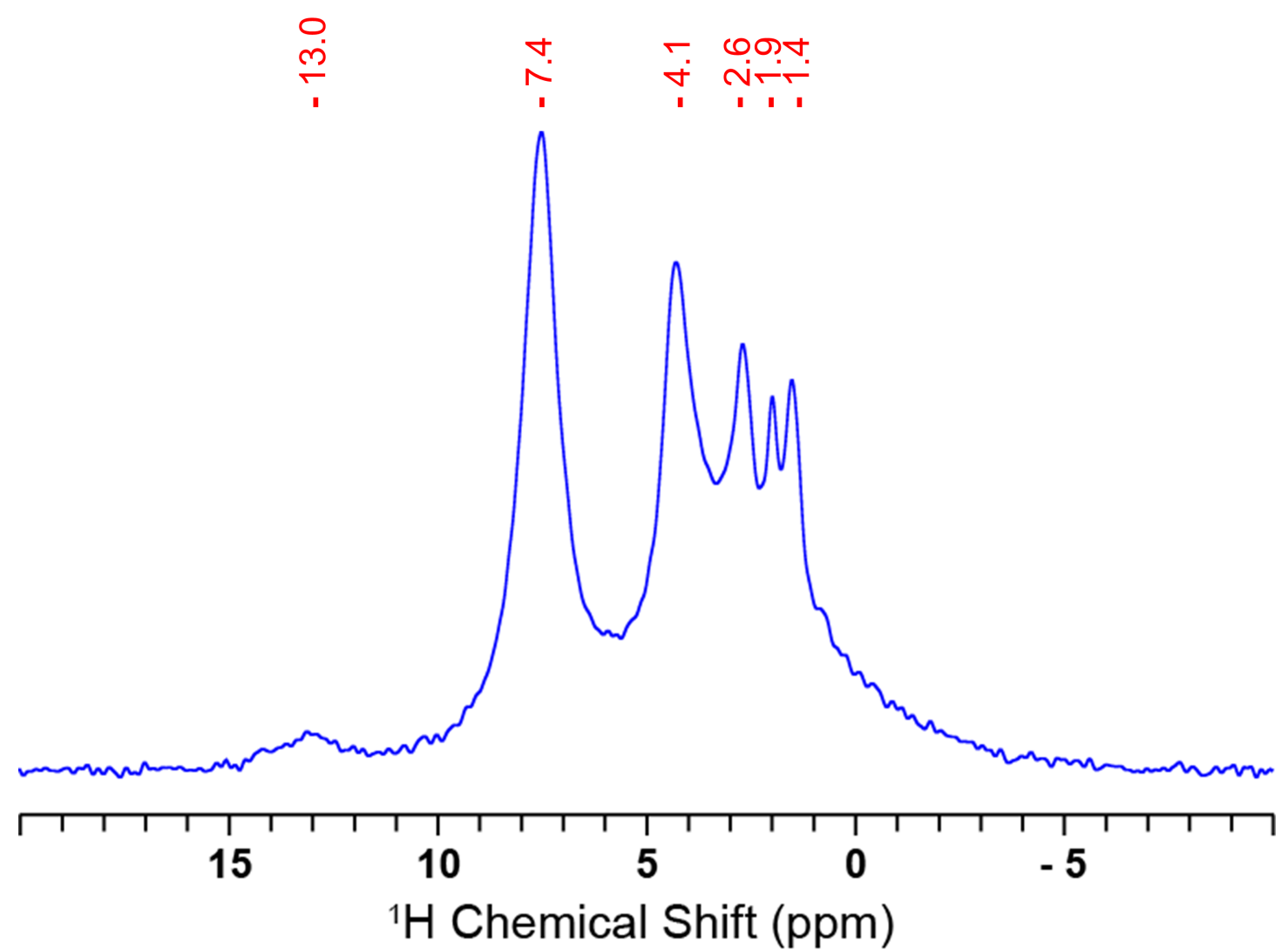

Figure S12. ${ }^{1} \mathrm{H}$ MAS NMR spectrum of $2 @ \mathrm{SiO}_{2}$, acquired at $9.4 \mathrm{~T}, 298 \mathrm{~K}, 10 \mathrm{kHz}$ MAS, with a 2 s recycle delay. 


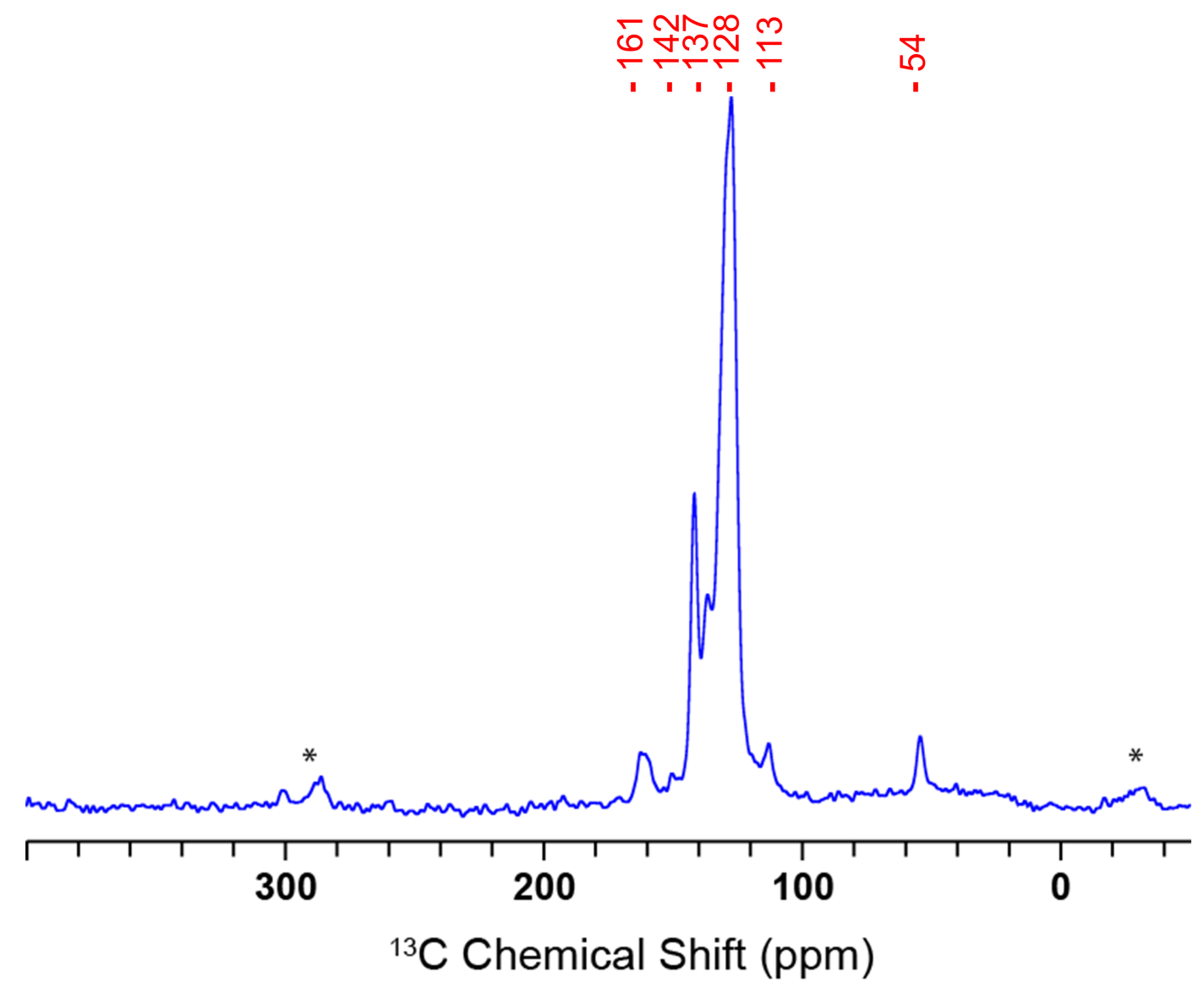

Figure S13. ${ }^{13} \mathrm{C}$ CP MAS NMR spectrum of $2 @ \mathrm{SiO}_{2}$, acquired at $9.4 \mathrm{~T}, 298 \mathrm{~K}, 10 \mathrm{kHz}$ MAS, with a $2 \mathrm{~s}$ recycle delay and with a ${ }^{13} \mathrm{C}-{ }^{1} \mathrm{H}$ contact time of $3 \mathrm{~ms}$. (* spinning side bands) 


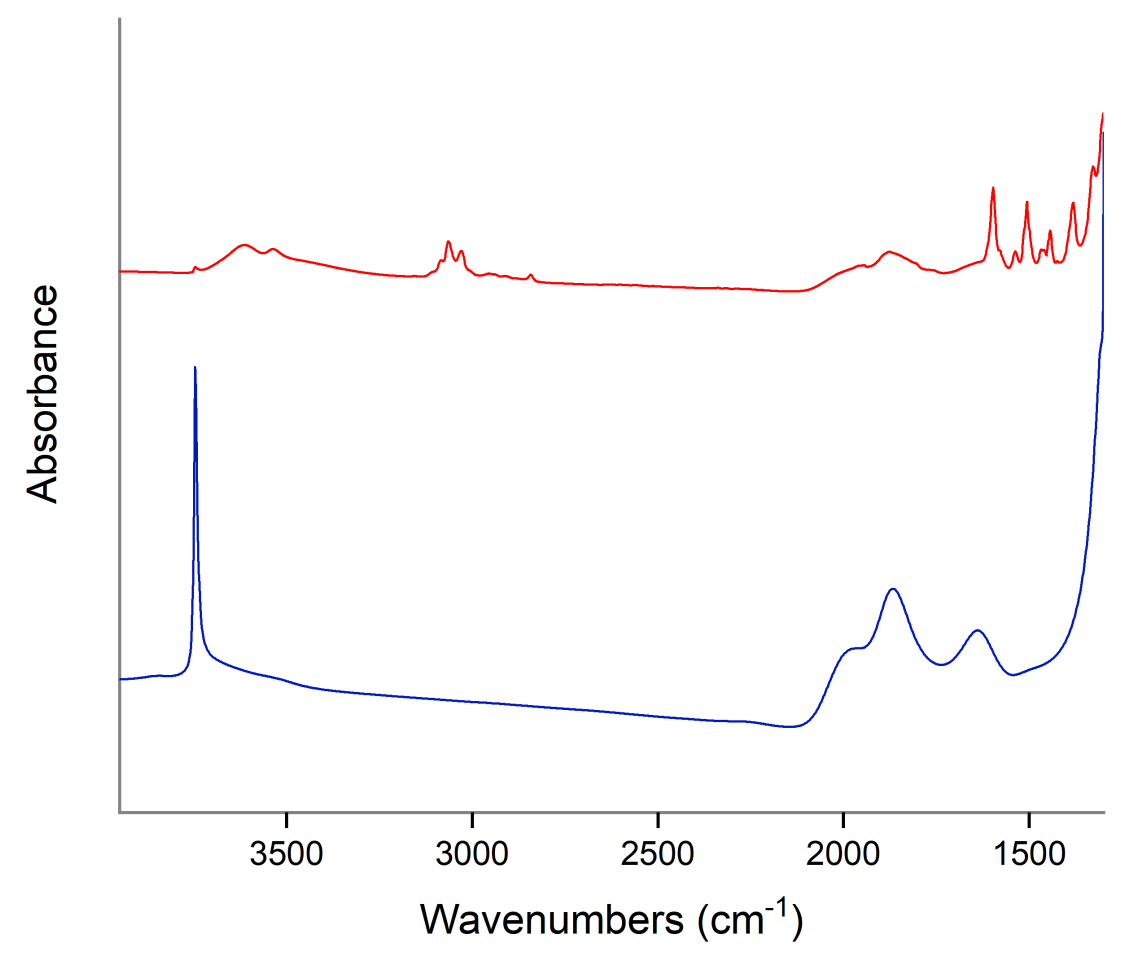

Figure S14. Transmission FT-IR spectra (from top) of $2 @ \mathrm{SiO}_{2}$ red, and $\mathrm{SiO}_{2-(700)}$ blue.

\section{$\left[(\equiv \mathrm{SiO}) \mathrm{Mo}(\mathrm{O})\left\{\mathrm{C} * \mathrm{H}-4-(\mathrm{MeO}) \mathrm{C}_{6} \mathrm{H}_{4}\right\}(\mathrm{THF})_{x}\left\{\mathrm{OC}\left(\mathrm{CF}_{3}\right)_{3}\right\}\right], 1 * @ \mathrm{SiO}_{2}$}

A red solution of $\mathbf{1}^{*}$ (10.9 $\mathrm{mg}, 0.014 \mathrm{mmol}, 1$ equiv.) was added to a suspension of $\mathrm{SiO}_{2-700}$ (45.6 mg, $0.013 \mathrm{mmol} \mathrm{SiOH}, 1$ equiv.) in cold toluene $\left(3 \mathrm{~mL},-30{ }^{\circ} \mathrm{C}\right)$ at $25^{\circ} \mathrm{C}$ in an Argon filled glovebox. The suspension was slowly stirred $(120 \mathrm{rpm})$ over $3 \mathrm{~h}$. The solid was collected by filtration and washed by suspension/filtration cycles in toluene $(3 \times 1 \mathrm{~mL})$, in benzene ( 3 $\mathrm{x} 1 \mathrm{~mL})$ and pentane $(3 \times 2 \mathrm{~mL})$. The resulting brown solid was dried thoroughly under high vacuum $\left(10^{-5} \mathrm{mbar}\right)$ at room temperature for 2 hours to afford $50 \mathrm{mg}$ of the titled compound. All filtrate solutions were collected and analyzed by ${ }^{19} \mathrm{~F}$ NMR spectroscopy in $\mathrm{C}_{6} \mathrm{D}_{6}$ using 4,4'-difluorobiphenyl as internal standard, indicating that $0.0076 \mathrm{mmol}$ of $\mathrm{HOC}\left(\mathrm{CF}_{3}\right)_{3}$ were released, corresponding to $58 \%$ grafting. 


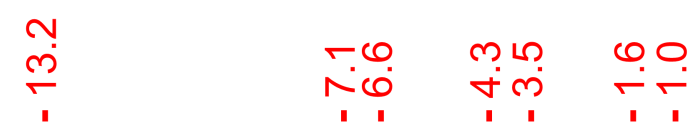

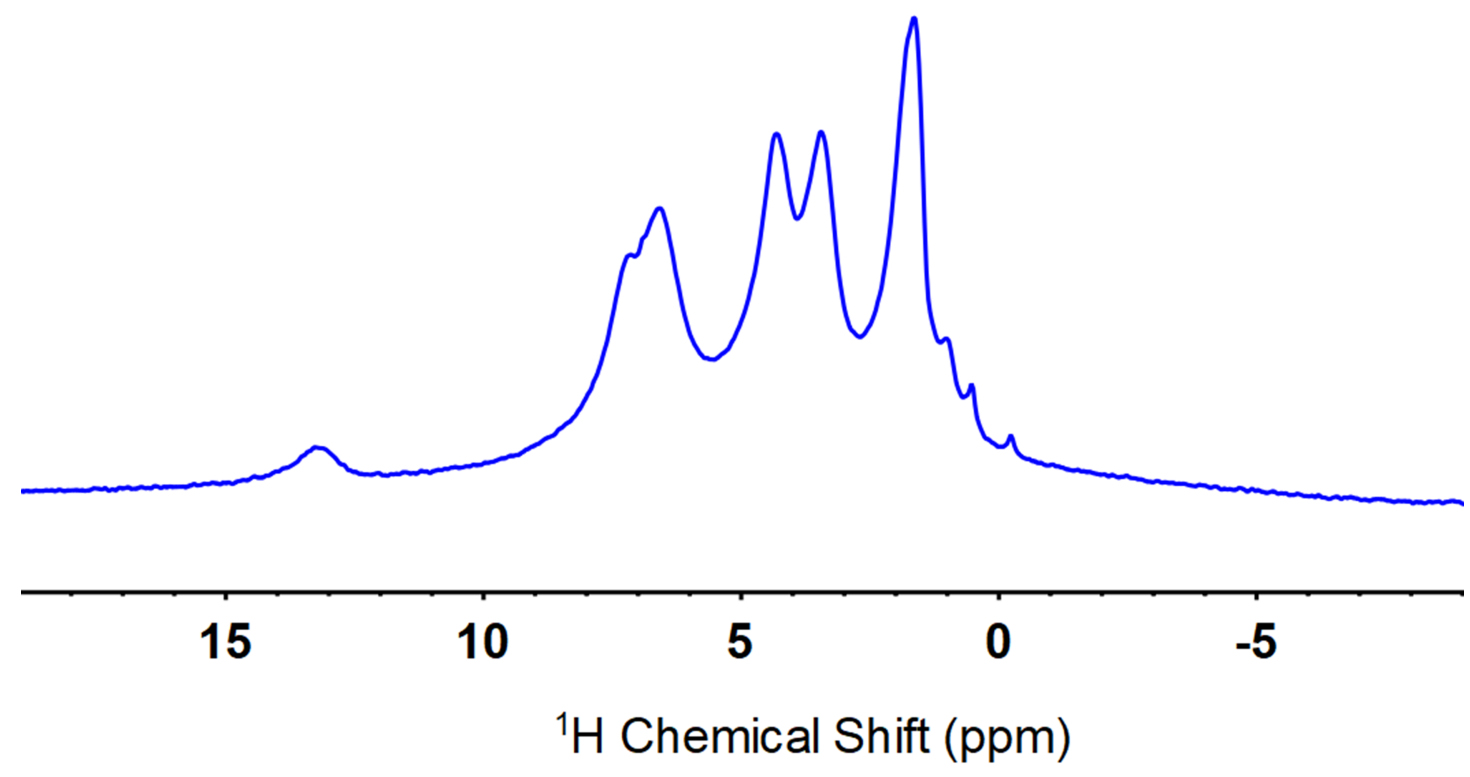

Figure S15. ${ }^{1} \mathrm{H}$ MAS NMR spectrum of $1 * @ \mathrm{SiO}_{2}$, acquired at $9.4 \mathrm{~T}, 270 \mathrm{~K}, 16 \mathrm{kHz}$ MAS, with a 1.5 s recycle delay. 


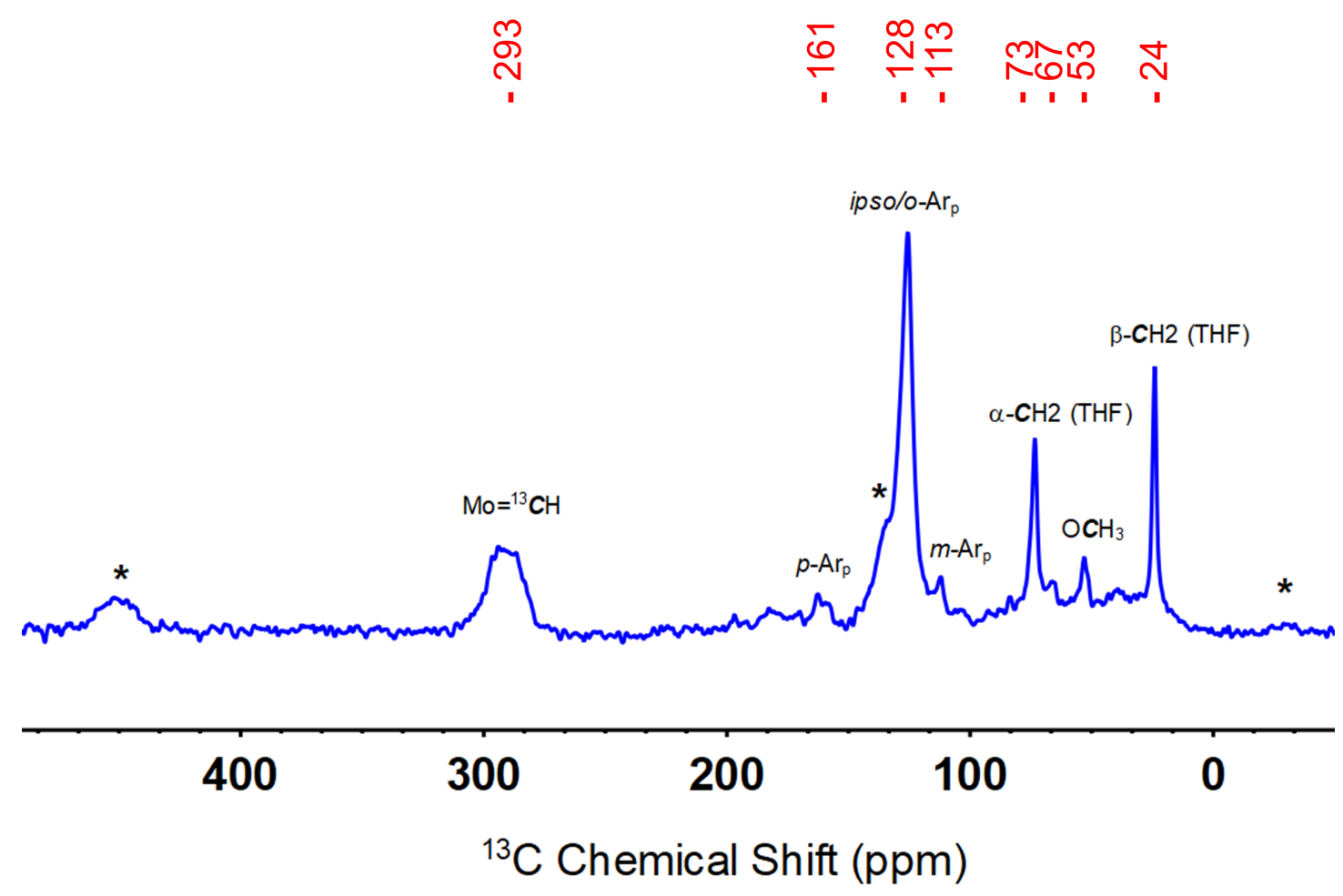

Figure S16. ${ }^{13} \mathrm{C}$ CP MAS NMR spectrum of $1 * @ \mathrm{SiO}_{2}$, acquired at $9.4 \mathrm{~T}, 270 \mathrm{~K}, 16 \mathrm{kHz}$ MAS, with a $1.5 \mathrm{~s}$ recycle delay and with a ${ }^{13} \mathrm{C}-{ }^{1} \mathrm{H}$ contact time of $3 \mathrm{~ms}$. (* spinning side bands) 


\section{DFT Calculations}

Conformational sampling of the ground state complexes was performed using $\mathrm{xtb} / \mathrm{crest}^{\left[{ }^{[4]}\right.}$ The geometry of the lowest energy conformer was optimized with the Gaussian09 package using the PBEPBE functional using the ultrafine integration grid and the D3 version of Grimme's dispersion with Becke-Johnson damping. ${ }^{[5,6]}$ Mo was represented by a SDD core potential and the associated basis sets. ${ }^{[7]}$ The remaining atoms $(\mathrm{H}, \mathrm{C}, \mathrm{N}, \mathrm{Si}, \mathrm{O}, \mathrm{F})$ were represented by a def2tzvp basis set. ${ }^{[8]}$ For the DFT calculations, the silica surface was modeled as a $\mathrm{O}-\mathrm{Si}(\mathrm{OMe})_{3}$ ligand. The electronic energies were calculated as a single point at $\mathrm{PBE} 1 \mathrm{PBE} \mathrm{E}^{[9]} / \mathrm{def} 2 \mathrm{tzvp}$ level of theory, with SDD core potential and associated basis sets for Mo. Reported are the thermal corrected Gibbs free energies and thermal corrected enthalpies in $\mathrm{kcal} / \mathrm{mol}$. 
a)

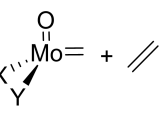

$\stackrel{O}{M 0}=+V$<smiles>[Y][Y10]1([Y])CCC1</smiles>

TBP

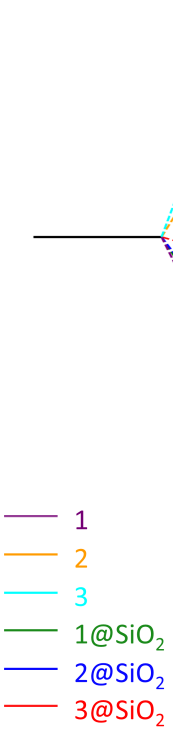

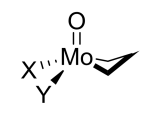

SP b)<smiles>[Y4][14C](=O)C=C</smiles>
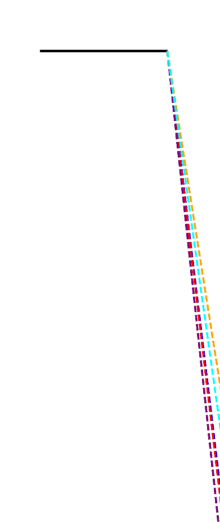

TBP<smiles>[Y][N+]1([Y])CCC1</smiles>

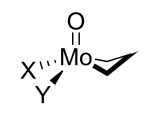

SP

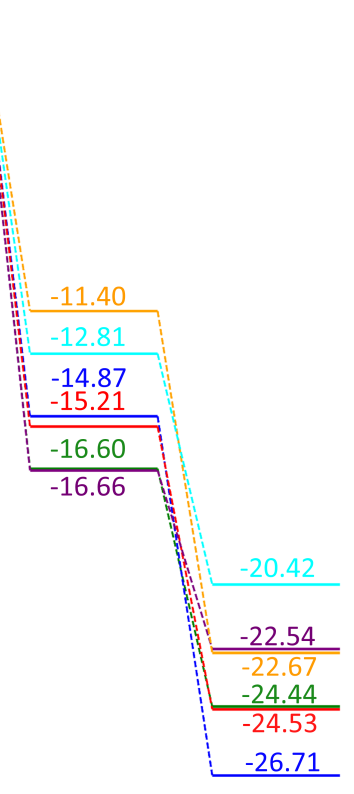

Turnstile

isomerization

c)

[2+2] cycloaddition
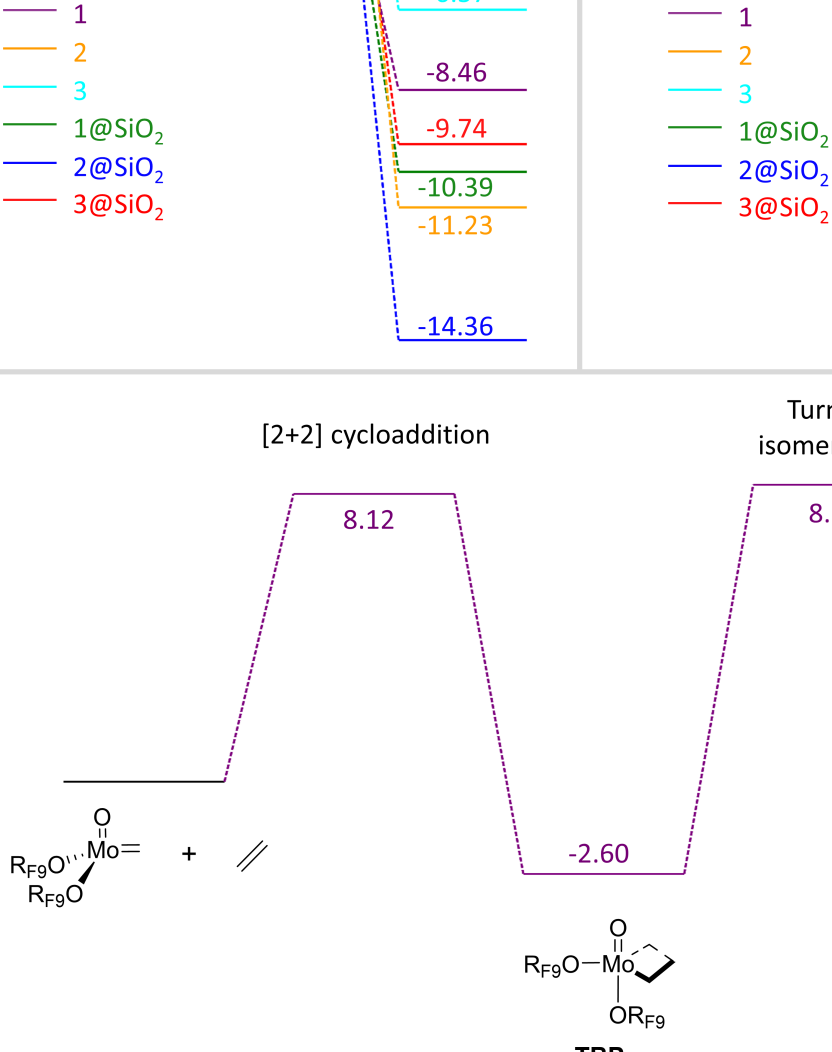

8.38

$-8.46$

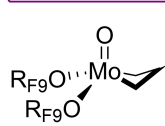

SP

Figure S17. Energy profiles for the ethylene metathesis of molecular and grafted oxo alkylidene complexes. a) Gibbs free energy profiles. b) Reaction enthalpy profiles. c) Gibbs free energy profile for $\mathbf{1}$, including [2+2]-cycloaddition and turnstile isomerization transition states. 


\section{Ethylene}

$\mathrm{H} \quad-0.9284680000 \quad 1.2408460000$

$\begin{array}{llll}\text { C } & 0.0000000000 & -0.6661060000 & 0.0000000000\end{array}$

$\begin{array}{llll}\text { C } & 0.0000000000 & 0.6661060000 & 0.0000000000\end{array}$

$\begin{array}{llll}\mathrm{H} & 0.9284610000 & 1.2408530000 & 0.0000000000\end{array}$

$\mathrm{H} \quad 0.9284680000 \quad-1.2408460000 \quad 0.0000000000$

\section{1-methylidene}

F $\quad-4.8648290000$

F $\quad-4.1622350000$

F $\quad-3.8912140000$

F $\quad-3.6407260000$

C $\quad-3.6163290000$

C $\quad-3.2628660000$

F $\quad-3.2489740000$

F $\quad-3.2140370000$

C $\quad-2.5966590000$

F $\quad-2.3248250000$

C $\quad-2.1716990000$

F $\quad-1.5735590000$

O $\quad-1.4757040000$

F $\quad-1.2681580000$

$\mathrm{H} \quad-0.6364500000$

$\mathrm{H} \quad-0.5749000000$

C $\quad-0.4567600000$

Mo 0.0148750000

O $\quad 0.2470570000$

O $\quad 1.4607300000$

F $\quad 1.5084760000$

F 1.6417550000

F 2.0825350000

C $\quad 2.5196030000$

C 2.6076960000

C 2.7345250000

F 3.1548740000

F $\quad 3.5721560000$

F $\quad 3.7145020000$

F $\quad 3.7147700000$

C $\quad 3.8454180000$

F 3.9195400000

$-0.3186940000$

1.9524360000

0.5816230000

0.2040780000

$-1.8924050000$

$-0.8159880000$

1.0532170000

$-1.2338350000$

1.6388810000

0.2531500000

1.7084280000

1.2467130000

2.3391240000

$-0.3550270000$

0.6386830000

$-3.5921360000$

$-1.9634020000$

$-2.5151990000$

$-1.4825990000$

$-2.6586740000$

$-0.3548450000$

0.2660330000

2.4050890000

1.9087670000

1.7732090000

0.2456540000

0.0977150000

$-1.1392000000$

0.9944600000

$-1.7724090000$

2.3859020000

$-0.4317190000$

$-0.0586220000$

$-0.1008820000$

F $\quad 5.0035440000$

\section{1-[2+2]-TS}

F $\quad-4.7951550000$
$-0.8015860000$

$-2.0899710000$

$-0.3119230000$

0.4928970000

$-1.2514310000$

1.7271070000

1.8341040000

$-0.0635680000$

$-1.9550730000$

1.0817270000

0.5733990000

$-0.5884310000$

1.8952980000

1.4480050000

2.3538450000

1.4102700000

$-0.0906490000$

$-1.2760470000$

0.5381960000

$-2.0620630000$

$-0.3538640000$

1.7182690000

0.4559150000

0.0671600000

$-1.4980060000$

$-1.8316250000$

$-2.0375230000$

0.7383640000

0.3485030000

0.7714290000

2.0634570000

0.1654280000

0.3767750000$$
\text { F } \quad-3.6629320000
$$$$
\text { C } \quad-3.5638230000
$$$$
-2.4366280000
$$

$-0.5220960000$

F $\quad-3.4961850000$

F $\quad-3.4312970000$

$-1.1211440000$

1.9306980000

F $\quad-3.4312970000$

0.3541300000

$-0.8206020000$

F $\quad-3.0343110000$

0.0683780000

$-2.1374090000$

C $\quad-2.6823490000$

1.6879890000

$-0.6855300000$

C $\quad-2.4279810000$

C $\quad-2.4184370000$

F $\quad-1.8569980000$

F $\quad-1.7271950000$

F $\quad-1.5603680000$

O -1.1848500000

$\quad-0.9701570000$

O $\quad-0.9007430000$

0.9686240000

1.8619750000

C $\quad-0.3401040000$

Mo -0.0384520000

H 0.0998570000

0.9829210000

H 0.9889960000

1.1115640000

1.4196310000

1.4424030000

C $\quad 1.5140890000$

F 1.7020920000

C $\quad 1.7877070000$

C 1.8081130000

$\mathrm{H} \quad 2.1249330000$

C $\quad 2.3751850000$

H 2.6278110000

C 2.7926020000

2.7953070000

3.3776100000

F $\quad 3.3789230000$

C $\quad 3.6325840000$

F $\quad 3.6597850000$

F $\quad 3.9255650000$

F $\quad 4.7275750000$

$-0.2963990000$

$-1.9299450000$

$-0.4026160000$

$-2.0811960000$

$-2.6684270000$

$-0.5949840000$

0.0924760000

3.3974070000

2.7451740000

2.5229970000

1.6870770000

2.0645880000

$-2.3909380000$

4.4324540000

$-1.9513760000$

0.3899510000

3.6001620000

3.4887630000

$-0.2638210000$

3.0267100000

$-1.9811260000$

3.1393750000

$-0.5388080000$

2.3554740000

$-0.4876890000$

$-2.8959750000$

$-0.5848370000$

$-1.6229670000$

$-0.2508800000$

0.5307950000

1.0727230000

1-TBP

F $\quad-4.8474320000 \quad-0.5751970000 \quad 0.3847540000$

F $\quad-4.0766330000 \quad 0.9497180000 \quad-0.9800210000$

C $\quad-3.8067070000 \quad 0.2388000000 \quad 0.1434630000$

F $\quad-3.7306730000 \quad 1.1312950000 \quad 1.1601660000$

F $\quad-3.5696620000 \quad-1.9215580000 \quad-1.6586760000$ 


\begin{tabular}{lccc} 
F & -3.0877730000 & -2.6395950000 & 1.0134310000 \\
F & -2.5811800000 & -0.9845370000 & 2.3517950000 \\
H & -2.5591740000 & 3.1441560000 & 0.2291080000 \\
C & -2.4371190000 & -0.5290070000 & -0.0100600000 \\
C & -2.4083230000 & -1.2849160000 & -1.3994770000 \\
C & -2.2797600000 & -1.5702830000 & 1.1682710000 \\
F & -2.1862140000 & -0.4005560000 & -2.3975100000 \\
H & -1.9148140000 & 2.2055170000 & -1.8028130000 \\
H & -1.4903110000 & 2.1461630000 & 2.0291720000 \\
C & -1.4884350000 & 3.3254710000 & 0.1075400000 \\
F & -1.4132920000 & -2.1890140000 & -1.4295570000 \\
O & -1.4067630000 & 0.3532940000 & 0.0391590000 \\
H & -1.2255760000 & 4.3860510000 & 0.0967100000 \\
C & -1.1003660000 & 2.7506660000 & -1.3254880000 \\
F & -1.0115650000 & -2.0015210000 & 1.2416500000 \\
C & -0.7914780000 & 2.7080190000 & 1.4080160000 \\
H & -0.6179680000 & 3.4912160000 & -1.9631060000 \\
H & -0.1920440000 & 3.4390180000 & 1.9501400000 \\
Mo & 0.1280870000 & 1.6367480000 & -0.0950770000 \\
O & 1.2465960000 & 0.0608370000 & -0.3909490000 \\
O & 1.3485230000 & 2.8228280000 & -0.2342770000 \\
F & 1.4712510000 & -2.5485280000 & -0.5299800000 \\
F & 1.8648770000 & -1.5872380000 & 1.9798010000 \\
F & 2.0986630000 & 0.5722680000 & 2.1074550000 \\
C & 2.4837160000 & -0.4214870000 & -0.0421330000 \\
C & 2.5939610000 & -0.5516210000 & 1.5239000000 \\
C & 2.6041640000 & -1.8508240000 & -0.7062900000 \\
F & 2.8116450000 & -1.7298530000 & -2.0349870000 \\
F & 3.3250410000 & 0.98175000000 & -1.7997130000 \\
F & 3.6244250000 & -2.5622950000 & -0.1805080000 \\
C & 3.6483300000 & 0.5057570000 & -0.5837980000 \\
F & 3.8507270000 & 1.55918000000 & 0.2359320000 \\
\hline & 3.8636330000 & -0.7112650000 & 1.9394430000 \\
F & 4.8169900000 & -0.1624600000 & -0.6880420000
\end{tabular}

\section{1-SP}

$\begin{array}{lrrr}\text { F } & -4.9361400000 & -0.4636850000 & -0.0887360000 \\ \text { F } & -3.9296940000 & 1.3649450000 & -0.7395200000 \\ \text { F } & -3.8862700000 & -0.4034810000 & -2.0057300000 \\ \text { C } & -3.8508090000 & 0.0189710000 & -0.7277250000 \\ \text { F } & -3.3255650000 & -2.7265800000 & -0.2192400000 \\ \text { F } & -3.3144210000 & 0.8515170000 & 1.8622530000 \\ \text { F } & -3.2591720000 & -1.3091920000 & 2.1152220000 \\ \text { C } & -2.6346230000 & -0.2698510000 & 1.5360660000 \\ \text { C } & -2.5162630000 & -0.4402810000 & -0.0294690000 \\ \text { C } & -2.2257090000 & -1.9623160000 & -0.3621720000 \\ \text { H } & -2.1104020000 & 3.1158770000 & -0.1405340000\end{array}$

$\begin{array}{lccc}\text { F } & -1.7834690000 & -2.0881860000 & -1.6233240000 \\ \text { H } & -1.6286600000 & 2.4670050000 & 1.4687350000 \\ \text { O } & -1.4675100000 & 0.2735080000 & -0.5620200000 \\ \text { F } & -1.3964460000 & -0.1641880000 & 2.0725580000 \\ \text { C } & -1.2678110000 & 2.9129180000 & 0.5336270000 \\ \text { F } & -1.2660680000 & -2.4342480000 & 0.4621500000 \\ \text { H } & -0.3763670000 & 4.6238210000 & 1.6524660000 \\ \text { C } & -0.2127820000 & 3.9769030000 & 0.7744830000 \\ \text { H } & -0.0953710000 & 4.6198160000 & -0.1090860000 \\ \text { Mo } & 0.0561770000 & 1.4562450000 & -0.4037650000 \\ \text { O } & 0.3339220000 & 2.2603480000 & -1.8591360000 \\ \text { C } & 0.9324900000 & 2.9874530000 & 0.8788930000 \\ \text { H } & 1.0258760000 & 2.5340640000 & 1.8753210000 \\ \text { O } & 1.3779800000 & 0.2889810000 & 0.4432840000 \\ \text { F } & 1.4226420000 & -2.3767010000 & -0.8978940000 \\ \text { F } & 1.5185260000 & -0.5679150000 & -2.1028510000 \\ \text { F } & 1.6805980000 & -1.9760910000 & 1.7592030000 \\ \text { H } & 1.9231420000 & 3.2603180000 & 0.4899070000 \\ \text { C } & 2.1968920000 & -1.3176980000 & -1.1965280000 \\ \text { C } & 2.4864470000 & -0.4478600000 & 0.0922440000 \\ \text { C } & 2.8079100000 & -1.3989430000 & 1.3104880000 \\ \text { F } & 3.3204560000 & -1.7628740000 & -1.7837440000 \\ \text { F } & 3.3460800000 & -0.7000820000 & 2.3305570000 \\ \text { F } & 3.5139330000 & 1.1730420000 & -1.3472430000 \\ \text { F } & 3.6765570000 & -2.3701800000 & 0.9590670000 \\ \text { C } & 3.7032280000 & 0.5180110000 & -0.1771580000 \\ \text { F } & 3.7920310000 & 1.4456750000 & 0.7938640000 \\ \text { F } & 4.8745780000 & -0.1395260000 & -0.2412820000\end{array}$

\section{1-turnstile-TS}

F $\quad-4.6722630000 \quad-0.0480730000 \quad-0.9203280000$

F $\quad-4.1903040000 \quad-1.0076720000 \quad 1.6836820000$

F $\quad-3.6015520000 \quad-2.5486380000 \quad-0.5465840000$

F $\quad-3.5132970000 \quad 1.0666630000 \quad 1.5971590000$

$\begin{array}{llll}\text { C } & -3.3586870000 & 0.2217110000 & -1.0677730000\end{array}$

F $\quad-3.2091020000 \quad 1.5674250000 \quad-1.0230160000$

$\begin{array}{llll}\text { C } & -3.1162000000 & -0.2261540000 & 1.4641820000\end{array}$

F $\quad-2.9763370000 \quad-0.1815810000 \quad-2.2952860000$

$\begin{array}{llll}\text { C } & -2.4715260000 & -0.4668270000 & 0.0409220000\end{array}$

C $\quad-2.3946270000 \quad-2.0241280000 \quad-0.2396970000$

F $\quad-2.2125760000 \quad-0.4630840000 \quad 2.4324820000$

$\mathrm{H} \quad-1.9463590000 \quad 3.1786760000 \quad 0.6969580000$

F $\quad-1.9199030000 \quad-2.6721150000 \quad 0.8437710000$

F $\quad-1.5603500000 \quad-2.2664680000 \quad-1.2650020000$

$\begin{array}{llll}\text { O } & -1.1929560000 & 0.0034780000 & 0.0376290000\end{array}$

$\mathrm{H} \quad-1.0365810000 \quad 2.4756230000 \quad 2.1091980000$

C $\quad-0.9908810000 \quad 2.8022340000 \quad 1.0653970000$ 


$\begin{array}{lccc}\text { O } & -0.3069100000 & 2.0633940000 & -1.7754650000 \\ \text { H } & -0.0319110000 & 4.4772400000 & -0.0015470000 \\ \text { Mo } & 0.0456660000 & 1.5610400000 & -0.2009330000 \\ \text { C } & 0.2445580000 & 3.7174470000 & 0.7346210000 \\ \text { H } & 0.5279390000 & 4.1723680000 & 1.6935830000 \\ \text { F } & 1.1627740000 & -2.5093710000 & 0.4353260000 \\ \text { O } & 1.2849240000 & 0.1243160000 & 0.3855790000 \\ \text { C } & 1.5492910000 & 2.9383950000 & 0.2825560000 \\ \text { F } & 1.5883560000 & -1.9680720000 & -1.6297860000 \\ \text { H } & 2.0601380000 & 3.4238050000 & -0.5513400000 \\ \text { C } & 2.0930500000 & -1.9867660000 & -0.3759220000 \\ \text { H } & 2.2190720000 & 2.7206170000 & 1.1158500000 \\ \text { F } & 2.4203100000 & 0.6052570000 & -2.0478380000 \\ \text { C } & 2.4549620000 & -0.5121010000 & 0.0799070000 \\ \text { F } & 2.8185870000 & -1.4252050000 & 2.2815730000 \\ \text { F } & 3.1694040000 & -2.8030600000 & -0.3661710000 \\ \text { C } & 3.2653270000 & 0.1983430000 & -1.0732780000 \\ \text { C } & 3.3371300000 & -0.5621700000 & 1.3879690000 \\ \text { F } & 3.3667970000 & 0.6567910000 & 1.9755890000 \\ \text { F } & 3.9057850000 & 1.2992480000 & -0.6133970000 \\ \text { F } & 4.1849340000 & -0.6093780000 & -1.6297820000 \\ \text { F } & 4.6092830000 & -0.9306030000 & 1.1321850000\end{array}$

\section{2-methylidene}

$\begin{array}{lrrr}\mathrm{H} & -9.1160190000 & 0.0890850000 & 0.4484640000 \\ \mathrm{H} & -8.1273930000 & -1.5590190000 & -1.1406910000 \\ \mathrm{C} & -8.0606670000 & 0.1521460000 & 0.1781180000 \\ \mathrm{H} & -7.6792840000 & 1.8865200000 & 1.4101590000 \\ \mathrm{C} & -7.5049720000 & -0.7731780000 & -0.7091220000 \\ \mathrm{C} & -7.2563930000 & 1.1593770000 & 0.7144800000 \\ \mathrm{C} & -6.1556940000 & -0.6993920000 & -1.0495500000 \\ \mathrm{C} & -5.9073500000 & 1.2369400000 & 0.3674840000 \\ \mathrm{H} & -5.7301560000 & -1.4262560000 & -1.7420110000 \\ \mathrm{C} & -5.3339470000 & 0.3062890000 & -0.5142940000 \\ \mathrm{H} & -5.2791350000 & 2.0189060000 & 0.7981650000 \\ \mathrm{H} & -4.7996920000 & -3.6355430000 & 2.2902780000 \\ \mathrm{H} & -4.6205680000 & -5.5779570000 & 0.7411910000 \\ \mathrm{C} & -4.3979920000 & -3.4982130000 & 1.2849190000 \\ \mathrm{C} & -4.2967990000 & -4.5875750000 & 0.4175890000 \\ \mathrm{H} & -4.0903320000 & 2.5155570000 & -1.3200200000 \\ \mathrm{H} & -4.0578960000 & -1.3815300000 & 1.5533730000 \\ \mathrm{C} & -3.9827430000 & -2.2324600000 & 0.8760300000 \\ \mathrm{C} & -3.9010880000 & 0.4183410000 & -0.8828250000 \\ \mathrm{H} & -3.8974260000 & 0.6543080000 & 3.4128380000 \\ \mathrm{C} & -3.7671520000 & -4.4068460000 & -0.8611230000 \\ \mathrm{H} & -3.6786890000 & -5.2540900000 & -1.5431980000 \\ \mathrm{C} & -3.4541020000 & -2.0363590000 & -0.4085600000\end{array}$

\begin{tabular}{|c|c|c|c|}
\hline & -3.4044680000 & 1.6681030000 & -1.2753050000 \\
\hline C & -3.3423880000 & -3.1429110000 & -1.2697790000 \\
\hline & -3.0363110000 & 2.2629960000 & 1.7107250000 \\
\hline C & -3.0274470000 & -0.6890550000 & -0.8603750000 \\
\hline & -2.9351800000 & -2.9994110000 & -2.2725480000 \\
\hline C & -2.8574410000 & 0.6037490000 & 3.0846530000 \\
\hline C & -2.3756500000 & 1.5 & 00 \\
\hline $\mathrm{H}$ & -2.3662780000 & 00 & 00 \\
\hline $\mathrm{H}$ & -2.265 & 00 & 0000 \\
\hline $\mathrm{C}$ & 950000 & 1.85529 & 0000 \\
\hline $\mathrm{C}$ & -2.0000970000 & -0.3706830000 & 3.6019490000 \\
\hline $\mathrm{C}$ & -1.8353460000 & 4.2999150000 & -1.2427390000 \\
\hline $\mathrm{C}$ & -1.6818750000 & -0.4877030000 & 610000 \\
\hline C & 340000 & 0000 & 0000 \\
\hline $\mathrm{H}$ & -1.5631780000 & 0000 & -0.9 \\
\hline C & 0390000 & 5.5849670000 & -1.610 \\
\hline C & -1.1942350000 & 0.7586340000 & 000 \\
\hline C & -1.0 & 3.4 & -3 \\
\hline $\mathrm{O}$ & -1.0 & -3.9 & 00 \\
\hline $\mathrm{C}$ & -1.0490750000 & 0000 & 00 \\
\hline $\mathrm{H}$ & -0.9434560000 & 2.6151180000 & 00000 \\
\hline $\mathrm{C}$ & -0.8625510000 & 5.8068160000 & -2.8648680000 \\
\hline $\mathrm{O}$ & -0.7782080000 & -1.4814230000 & -1.1044340000 \\
\hline $\mathrm{C}$ & -0.6961330000 & 4.7357670000 & -3.7474000000 \\
\hline $\mathrm{H}$ & -0.6840450000 & 2.1544690000 & 0.9757670000 \\
\hline $\mathrm{C}$ & -0.6725830000 & -0.4357870000 & 3.1790810000 \\
\hline $\mathrm{H}$ & -0.5 & 6.8 & 00 \\
\hline $\mathrm{H}$ & -( & 0 & 00 \\
\hline $\mathrm{C}$ & -( & 0 & 00 \\
\hline Mo & 0 & 0 & 000 \\
\hline H & -0.0091010000 & -1.1974280000 & 3.5893460000 \\
\hline $\mathrm{H}$ & 0.1050640000 & 4.3402190000 & 5.0427940000 \\
\hline $\mathrm{H}$ & 0.1357010000 & -0.7911170000 & -3.4791610000 \\
\hline $\mathrm{C}$ & 0.2263970000 & 0.8773480000 & -2.1112830000 \\
\hline$H$ & 0.3440460000 & 6.3701450000 & 3.6147730000 \\
\hline $\mathrm{C}$ & 0.5592130000 & 4.2627820000 & 4.0533400000 \\
\hline $\mathrm{H}$ & 0.6617160000 & 2.5688960 & -0.8414520 \\
\hline C & 0.6963390000 & 5.4019360000 & 3.2550050 \\
\hline C & 0.7670920000 & -0.0054700000 & -3.0 \\
\hline $\mathrm{C}$ & 0.8570200000 & -3.9692310000 & -1.0354690000 \\
\hline $\mathrm{H}$ & 0.8780250000 & 2.1360080000 & 4.2151510000 \\
\hline $\mathrm{H}$ & 0.9155620000 & -5.0594200000 & -0.9955710000 \\
\hline $\mathrm{O}$ & 0.9829520000 & -1.8490700000 & 1.2778120000 \\
\hline$C$ & 0.9971730000 & 3.0231340000 & 3.5918960000 \\
\hline $\mathrm{C}$ & 0000 & 1.87 & 350000 \\
\hline & 1.2485690000 & 0.4386950000 & 1.8011140000 \\
\hline
\end{tabular}




\begin{tabular}{|c|c|c|c|}
\hline & 50000 & 000 & 0 \\
\hline H & 0000 & 0000 & 0000 \\
\hline $\mathrm{H}$ & 1.5435640000 & .4696600000 & 7340130000 \\
\hline $\mathrm{C}$ & 1.5901250000 & 2.8985480000 & 2.3250380000 \\
\hline $\mathrm{C}$ & 1.7394040000 & 4.0532940000 & 1.5402180000 \\
\hline $\mathrm{C}$ & 1.7968330000 & -0.7663000000 & 1.2941500000 \\
\hline $\mathrm{C}$ & 2.0665970000 & 1.5893280000 & 1.8111270000 \\
\hline $\mathrm{C}$ & 2.0900460000 & 0.1193070000 & -3.4830180000 \\
\hline $\mathrm{H}$ & 2.1921740000 & 3.9699750000 & 0.5502840000 \\
\hline $\mathrm{C}$ & 2.3810580000 & 1.9986950000 & -2.0016910000 \\
\hline $\mathrm{H}$ & 2.4874140000 & -0.5729710000 & -4.2277220000 \\
\hline $\mathrm{C}$ & 2.9043490000 & 1.1251000000 & -2.9555090000 \\
\hline $\mathrm{H}$ & 3.0071760000 & 2.7843140000 & -1.5757640000 \\
\hline $\mathrm{C}$ & 3.1140360000 & -0.8472550000 & 0.7946440000 \\
\hline $\mathrm{H}$ & 3.2203780000 & -3.3226540000 & 1.9360670000 \\
\hline $\mathrm{C}$ & 3.3786880000 & 1.5021940000 & 1.3331090000 \\
\hline $\mathrm{C}$ & 3.5912800000 & -3.3166130000 & 0.9101190000 \\
\hline $\mathrm{C}$ & 3.5931640000 & -2.1139600000 & 0.1878050000 \\
\hline $\mathrm{C}$ & 3.9199830000 & 0.3132950000 & 0.8325420000 \\
\hline $\mathrm{H}$ & 3.9422390000 & 1.2213790000 & -3.2793180000 \\
\hline $\mathrm{H}$ & 4.0196710000 & 0000 & 30000 \\
\hline $\mathrm{H}$ & 4.0511670000 & -1.1954 & 280000 \\
\hline $\mathrm{H}$ & 4.0552860000 & -5.4209650000 & 0.9075300000 \\
\hline $\mathrm{C}$ & 4.0601170000 & -4.4950090000 & 0.3296960000 \\
\hline $\mathrm{C}$ & 4.0602260000 & -2.1243630000 & -1.1360370000 \\
\hline $\mathrm{C}$ & 4.5236760000 & -3.3034520000 & -1.7182950000 \\
\hline $\mathrm{C}$ & 4.5295180000 & -4.4929970000 & -0.9860120000 \\
\hline $\mathrm{H}$ & 4.8829390000 & -3.2926350000 & -2.7490860000 \\
\hline $\mathrm{H}$ & 4.8944050000 & -5.4160020000 & -1.4398050000 \\
\hline $\mathrm{H}$ & 5.1322770000 & 2.1139640000 & -0.7745130000 \\
\hline $\mathrm{C}$ & 5.3379340000 & 0.3125670000 & 0.3938550000 \\
\hline $\mathrm{C}$ & 5.8214210000 & 1.3405650000 & -0.4320090000 \\
\hline $\mathrm{H}$ & 5.8852090000 & -1.4796670000 & 1.4680870000 \\
\hline $\mathrm{C}$ & 6.2385490000 & -0.6791990000 & 0.8175980000 \\
\hline $\mathrm{C}$ & 7.1566440000 & 1.3703050000 & -0.8352810000 \\
\hline $\mathrm{H}$ & 7.5080520000 & 2.1734070000 & -1.4858630000 \\
\hline $\mathrm{C}$ & 7.5739350000 & -0.6467710000 & 0.4209920000 \\
\hline $\mathrm{C}$ & 8.0385960000 & 0.3744090000 & -0.4119730000 \\
\hline $\mathrm{H}$ & 8.2575780000 & -1.4242740000 & 0.7665550000 \\
\hline $\mathrm{H}$ & 9.0834030000 & 0.3949980000 & -0.7263540000 \\
\hline
\end{tabular}

\section{2-TBP}

$\begin{array}{lrrr}\mathrm{H} & -9.7240850000 & 0.1261200000 & 0.0336700000 \\ \mathrm{C} & -8.6464180000 & 0.1993400000 & -0.1220980000 \\ \mathrm{H} & -8.4987480000 & 2.1954070000 & 0.6932830000 \\ \mathrm{H} & -8.4728190000 & -1.7729320000 & -0.9896490000 \\ \mathrm{C} & -7.9602210000 & 1.3583580000 & 0.2449500000\end{array}$

\begin{tabular}{|c|c|c|c|}
\hline & .9438380000 & 0000 & -0.69208100 \\
\hline & .5821970000 & 1.4489830000 & 044 \\
\hline & .5665930000 & -0.7777070000 & -0.8846110000 \\
\hline & -6.0450990000 & 2.3517670000 & .3431860000 \\
\hline & -6.0234390000 & -1.6135200000 & -1.32 \\
\hline & 0000 & 0 & 30000 \\
\hline & 0000 & -2.4 & 0000 \\
\hline & -5.1058620000 & -4.72 & 00000 \\
\hline & 0 & -2.57 & 00 \\
\hline & 0 & -( & 1.9594720000 \\
\hline & -4 & -3.8 & 000 \\
\hline & -4.67 & 00 & 0000 \\
\hline & -4.6416880000 & -1.4794 & 0000 \\
\hline & -4.4023470000 & 0.5082420000 & -0.7505160000 \\
\hline & -4.0561380000 & -4.0646900000 & 0000 \\
\hline & 960000 & 0000 & 0000 \\
\hline & -3.91 & 0 & 00 \\
\hline & 0 & 0 & 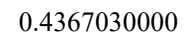 \\
\hline & 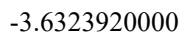 & -2.9706740000 & 0.0284880000 \\
\hline & . & 0 & \\
\hline & -3.2 & -0.4 & 4.0636400000 \\
\hline & -3.20 & 00 & -1 \\
\hline & -3.092 & -3.1 & -0.9 \\
\hline $\mathrm{H}$ & -2.85 & 1.583 & 2.70 \\
\hline & -2.6344060000 & 4.3011300000 & 810000 \\
\hline & -2.6280450000 & 6.4310150000 & -2.4195720000 \\
\hline & -2.5991400000 & 1.8211570000 & -1.76 \\
\hline & -2.3032180000 & 5.45711400 & -2.7905 \\
\hline $\mathrm{C}$ & -2.2785170000 & -0.3150700000 & 0 \\
\hline & -2.21 & 2. & -2.5301600000 \\
\hline & & & \\
\hline & & & o \\
\hline C & -1 & 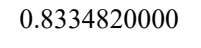 & 0 \\
\hline $\mathrm{C}$ & -1.554 & U & 00 \\
\hline $\mathrm{C}$ & -1.4677650000 & 2.9622280000 & -3.7157100000 \\
\hline $\mathrm{H}$ & -1.4373240000 & -2.1848750000 & 4.2485290000 \\
\hline $\mathrm{H}$ & -1.2937930000 & 6.2716460000 & -4.5204460000 \\
\hline $\mathrm{C}$ & -1.2681830000 & -1.273113 & 50000 \\
\hline $\mathrm{O}$ & -1.204 & -1.1358760000 & -014 \\
\hline & -1.14 & 0 & -40 \\
\hline $\mathrm{C}$ & -1.1423710000 & 0 & -4.4267650 \\
\hline $\mathrm{O}$ & -1.0490320000 & -3.1396300000 & -1.8900040000 \\
\hline$c$ & -0.8331130000 & 1.0465090000 & 2.1903610000 \\
\hline & -0.6669010000 & 1.9523980000 & 1.6077850000 \\
\hline & -0.5641520000 & 4.0355020000 & -5.3490540000 \\
\hline 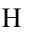 & -0.4518670000 & -5.1108360000 & 0.1639790000 \\
\hline
\end{tabular}




\begin{tabular}{|c|c|c|c|}
\hline & 00 & 00 & 0 \\
\hline & 000 & 000 & 00 \\
\hline & -0.0424530000 & -1.0659640000 & 3.0436950000 \\
\hline & -0.0034020000 & 2.8170370000 & -0.5745900000 \\
\hline & 0.0219080000 & -2.5380410000 & -0.6759340000 \\
\hline C & 0.1981760000 & 0.1030610000 & 2.3041760000 \\
\hline $\mathrm{C}$ & 0.3269990000 & -4.3506660000 & 0.2327790000 \\
\hline & 0.3515440000 & 5.5190250000 & 5.1005230000 \\
\hline & 0.4280640000 & 3.1881060000 & 5.9828340000 \\
\hline $\mathrm{C}$ & 0.4439400000 & 0.0661670000 & -2.5255480000 \\
\hline C & 0.5017130000 & 2.0892470000 & -1.2111420000 \\
\hline $\mathrm{C}$ & 0.7339710000 & 4.7088450000 & 4.4773800000 \\
\hline $\mathrm{H}$ & 0.7522070000 & -1.8084520000 & 200000 \\
\hline $\mathrm{C}$ & 0.7745450000 & 3.4016270000 & 4.9700940000 \\
\hline $\mathrm{H}$ & 0.7869930000 & -4.2848380000 & 1.2222120000 \\
\hline $\mathrm{H}$ & 1.0875340000 & -5.2853550000 & -1.5804920000 \\
\hline $\mathrm{H}$ & 1.1582140000 & 5.9885550000 & 2.7879720000 \\
\hline $\mathrm{C}$ & 1.1874960000 & 4.9715970000 & 3.1836010000 \\
\hline $\mathrm{C}$ & 1.2535270000 & 2.3636040000 & 4.1733520000 \\
\hline $\mathrm{H}$ & 1.2734870000 & 1.3437700000 & 4.5593610000 \\
\hline $\mathrm{H}$ & 1.35 & -3.4 & 0000 \\
\hline $\mathrm{C}$ & 1.4002910000 & -4.4502780000 & -0.9478170000 \\
\hline $\mathrm{O}$ & 1.4167730000 & -1.6599480000 & 0000 \\
\hline $\mathrm{C}$ & 1.5426460000 & 0.3466 & 30000 \\
\hline $\mathrm{C}$ & 1.5963330000 & -3.1918500000 & 00000 \\
\hline $\mathrm{C}$ & 1.6758050000 & 3.9327880000 & 2.3894890000 \\
\hline $\mathrm{C}$ & 1.7090610000 & 2.6142200000 & 2.8692050000 \\
\hline $\mathrm{C}$ & 1.7915350000 & 0.2396150000 & -2.8514660000 \\
\hline $\mathrm{C}$ & 1.8428670000 & 2.2655320000 & -1.5415340000 \\
\hline $\mathrm{H}$ & 2.0212100000 & 4.1371160000 & 1.3737240000 \\
\hline $\mathrm{C}$ & 2.1517930000 & -0.6302300000 & 0.8947530000 \\
\hline $\mathrm{C}$ & 2.2556660000 & 1.530 & 2.011 \\
\hline $\mathrm{H}$ & 2.2905690000 & -0.4898030000 & -3.4917220000 \\
\hline $\mathrm{H}$ & 000 & -4.620 & 00 \\
\hline $\mathrm{H}$ & 000 & 0 & 00 \\
\hline $\mathrm{C}$ & +50000 & 70000 & 0000 \\
\hline $\mathrm{H}$ & 2.5619940000 & -2.7110530000 & -1.7427590000 \\
\hline $\mathrm{C}$ & 3.5063080000 & -0.5022740000 & 0.4864050000 \\
\hline C & 3.5434950000 & 1.7013550000 & 1.4925660000 \\
\hline $\mathrm{H}$ & 3.5438130000 & 1.4850420000 & -2.6195150000 \\
\hline $\mathrm{H}$ & 3.8006 & -3.02 & 1.37 \\
\hline $\mathrm{H}$ & 00 & 2.609 & 1.74 \\
\hline$\theta$ & 4.1773910000 & -1.6208360000 & -0.2185760000 \\
\hline 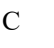 & 4.1946910000 & 0.6941450000 & 0.7732130000 \\
\hline $\mathrm{C}$ & 4.2612480000 & -2.8813960000 & 0.3961500000 \\
\hline & 4.7477910000 & -0.4706280000 & -1.9498580000 \\
\hline
\end{tabular}

$\begin{array}{lrrr}\mathrm{C} & 4.7935800000 & -1.4467390000 & -1.4680210000 \\ \mathrm{C} & 4.9393470000 & -3.9343140000 & -0.2188540000 \\ \mathrm{H} & 5.0084970000 & -4.9017900000 & 0.2829760000 \\ \mathrm{H} & 5.1378410000 & 2.7990880000 & -0.6104760000 \\ \mathrm{C} & 5.4586890000 & -2.5021250000 & -2.0908620000 \\ \mathrm{C} & 5.5348110000 & -3.7509350000 & -1.4694380000 \\ \mathrm{C} & 5.6023170000 & 0.9309890000 & 0.3626440000 \\ \mathrm{H} & 5.9257980000 & -2.3466320000 & -3.0649140000 \\ \mathrm{C} & 5.9319000000 & 2.0972030000 & -0.3467590000 \\ \mathrm{H} & 6.0628070000 & -4.5745000000 & -1.9527160000 \\ \mathrm{H} & 6.3960630000 & -0.8696320000 & 1.2519230000 \\ \mathrm{C} & 6.6330480000 & 0.0362640000 & 0.6927550000 \\ \mathrm{C} & 7.2488250000 & 2.3558320000 & -0.7293930000 \\ \mathrm{H} & 7.4823320000 & 3.2635690000 & -1.2889220000 \\ \mathrm{C} & 7.9497120000 & 0.2971930000 & 0.3172790000 \\ \mathrm{C} & 8.2630240000 & 1.4546790000 & -0.4002000000 \\ \mathrm{H} & 8.7376830000 & -0.4076630000 & 0.5886790000 \\ \mathrm{H} & 9.2937170000 & 1.6545820000 & -0.6977780000\end{array}$

\section{2-SP}

$\begin{array}{lrrr}\mathrm{H} & -9.5366780000 & 0.1664520000 & -2.3927110000 \\ \mathrm{H} & -8.6308580000 & -1.9989000000 & -1.5513650000 \\ \mathrm{C} & -8.5698270000 & 0.1353500000 & -1.8876840000 \\ \mathrm{H} & -8.2236630000 & 2.2611790000 & -2.0668360000 \\ \mathrm{C} & -8.0595980000 & -1.0783210000 & -1.4193260000 \\ \mathrm{C} & -7.8353200000 & 1.3081270000 & -1.7030390000 \\ \mathrm{C} & -6.8216070000 & -1.1219150000 & -0.7809980000 \\ \mathrm{C} & -6.5992210000 & 1.2648810000 & -1.0571190000 \\ \mathrm{H} & -6.4283670000 & -2.0728780000 & -0.4200180000 \\ \mathrm{C} & -6.0707660000 & 0.0498130000 & -0.5923200000 \\ \mathrm{H} & -6.0214090000 & 2.1817950000 & -0.9218990000 \\ \mathrm{H} & -5.3875280000 & 1.5978220000 & 1.4465880000 \\ \mathrm{C} & -4.7641450000 & 0.0324820000 & 0.1126100000 \\ \mathrm{H} & -4.5973710000 & -1.9101070000 & -4.6971330000 \\ \mathrm{C} & -4.5701690000 & 0.9313600000 & 1.1675730000 \\ \mathrm{H} & -4.3708310000 & -0.3084380000 & -2.8215940000 \\ \mathrm{C} & -4.3339950000 & -2.2751040000 & -3.7028530000 \\ \mathrm{H} & -4.2624850000 & -4.3508440000 & -4.3058730000 \\ \mathrm{C} & -4.1939670000 & -1.3712370000 & -2.6506250000 \\ \mathrm{C} & -4.1474120000 & -3.6420870000 & -3.4842130000 \\ \mathrm{C} & -3.8523480000 & -1.8129920000 & -1.3623720000 \\ \mathrm{H} & -3.8308070000 & 3.5881730000 & 1.8687970000 \\ \mathrm{C} & -3.8145130000 & -4.0954230000 & -2.2061850000 \\ \mathrm{H} & -3.7498350000 & 5.2081920000 & 3.7520990000 \\ \mathrm{C} & -3.7287600000 & -0.8628170000 & -0.2297930000 \\ \mathrm{H} & -3.6670080000 & -5.1614750000 & -2.0245500000 \\ \mathrm{C} & -3.6621110000 & -3.1883020000 & -1.1579320000\end{array}$




\begin{tabular}{|c|c|c|c|}
\hline & 00 & 00 & \\
\hline & 0 & 0 & 0000 \\
\hline & .4069710000 & 9329420000 & 453320000 \\
\hline & -3.4015220000 & -3.5458040000 & -0.1603730000 \\
\hline c & -3.3272920000 & 1.8804300000 & 3.0856740000 \\
\hline C & -3.2327400000 & 3.7110440000 & 5.2225280000 \\
\hline $\mathrm{H}$ & -3.1920030000 & 4.4200880000 & 6.0510530000 \\
\hline $\mathrm{H}$ & -3.1796500000 & 2.6477940000 & -1.4477410000 \\
\hline $\mathrm{C}$ & -3.0158290000 & 1.4498020000 & 4.3852140000 \\
\hline $\mathrm{C}$ & -2.9723 & 2.3560280000 & 50000 \\
\hline $\mathrm{H}$ & -2.8085800000 & 0.3941340000 & 4.5640910000 \\
\hline $\mathrm{H}$ & -2.7336910000 & 2.0015080000 & 6.4473920000 \\
\hline $\mathrm{C}$ & -2.5244180000 & -0.8141650000 & 0000 \\
\hline $\mathrm{C}$ & -2.3663920000 & 0.0314350000 & 1.6370030000 \\
\hline $\mathrm{H}$ & -2.3098480000 & 0.6230300000 & -2.6161180000 \\
\hline $\mathrm{C}$ & -2.1280050000 & 2.3712910000 & -1.3574410000 \\
\hline $\mathrm{C}$ & -1.6444990000 & 1.2353970000 & -2.0076920000 \\
\hline $\mathrm{H}$ & -1.6264310000 & 4.0325560000 & -0.0679920000 \\
\hline $\mathrm{O}$ & -1.4676940000 & -1.5062800000 & 0.0484210000 \\
\hline $\mathrm{H}$ & -1.31 & 0000 & 80000 \\
\hline $\mathrm{C}$ & -1.25 & 00 & 0000 \\
\hline $\mathrm{C}$ & 0000 & 0000 & 0000 \\
\hline $\mathrm{H}$ & -0.9682040000 & -3.0785 & 0000 \\
\hline $\mathrm{H}$ & -0.8544010000 & -4.464 & -0.97 \\
\hline $\mathrm{C}$ & -0.7039140000 & -1.200 & 50000 \\
\hline $\mathrm{H}$ & -0.6299960000 & 2.0676910000 & 2.1144110000 \\
\hline $\mathrm{C}$ & -0.3286030000 & 1.1423320000 & 2.6031120000 \\
\hline $\mathrm{C}$ & -0.3057670000 & 0.8676650000 & -1.8760060000 \\
\hline $\mathrm{C}$ & -0.3056860000 & -3.6333970000 & -1.4450150000 \\
\hline $\mathrm{H}$ & 0.0659000000 & -0.0131240000 & -2.4029370000 \\
\hline $\mathrm{C}$ & 0.0819070000 & 2.7796290000 & -0.4627980000 \\
\hline M & 0.32382 & -2.1978230 & 0.06 \\
\hline $\mathrm{C}$ & 0.442581 & -1.2359550000 & 3.84280 \\
\hline $\mathrm{C}$ & 0. & 1. & -1 . \\
\hline $\mathrm{H}$ & 000 & -2 & 00 \\
\hline $\mathrm{H}$ & 0.763 & 000 & 0000 \\
\hline $\mathrm{C}$ & 0.8193150000 & 1.1057390000 & 3.3899760000 \\
\hline $\mathrm{H}$ & 0.8296510000 & 4.5514420000 & -4.8516520000 \\
\hline $\mathrm{O}$ & 0.8485060000 & -3.3658690000 & 1.1843380000 \\
\hline $\mathrm{C}$ & 1.0689390000 & -3.8882860000 & -2.0341750000 \\
\hline $\mathrm{H}$ & 1.0956040000 & -4.1431 & -3.1085860000 \\
\hline $\mathrm{C}$ & 1.203 & -0.0805 & 0 \\
\hline $\mathrm{H}$ & 1.3027900000 & 4650060000 & -3.607 \\
\hline $\mathrm{H}$ & 1.4030140000 & -1.7458060000 & -2.3842330000 \\
\hline $\mathrm{H}$ & 1.4181270000 & 2.0097730000 & 3.5140900000 \\
\hline c & 1.4623770000 & -0.6546830000 & 0.2797760000 \\
\hline
\end{tabular}

\begin{tabular}{|c|c|c|c|}
\hline & 1.5296230000 & 4.5686020000 & -4.014 \\
\hline & 1.5878140000 & -4.6868360000 & -1.4841670000 \\
\hline & 1.6369800000 & -2.5329990000 & -1.6509100000 \\
\hline C & 1.7952870000 & 3.3924910000 & -3.3154250000 \\
\hline & 1.9412400000 & 6.6845420000 & -4.1930840000 \\
\hline & 2.0178570000 & 1.2832410000 & -0.9873610000 \\
\hline & 2.0972680000 & -0.1041800000 & 4.6482040000 \\
\hline & 2.1548310000 & 5.7632700000 & -3.6485060000 \\
\hline & 2.4134960000 & 0.1220200000 & -0.2835780000 \\
\hline & 2.6912760000 & -2.4625230000 & -1.3587900000 \\
\hline & 2.6964080000 & 3.3854050000 & 00000 \\
\hline & 2.9756190000 & -1.0335850000 & 380000 \\
\hline C & 3.0173420000 & 2.1391640000 & -1.4968510000 \\
\hline $\mathrm{C}$ & 3.0555560000 & 5.7696350000 & -2.5818970000 \\
\hline $\mathrm{C}$ & 3.3260850000 & 4.5906740000 & -1.8865370000 \\
\hline $\mathrm{H}$ & 3.5473460000 & 6.6978950000 & -2.2852650000 \\
\hline $\mathrm{H}$ & 3.5980630000 & -3.1274050000 & 3.5534510000 \\
\hline C & 3.64 & -1 & 1.90 \\
\hline $\mathrm{C}$ & 3.77 & -0.2 & 000 \\
\hline $\mathrm{C}$ & 3.9984840000 & -2.5 & 2.5 \\
\hline $\mathrm{H}$ & 0000 & 0000 & 000 \\
\hline $\mathrm{C}$ & 4.1413440000 & -1.4628 & 0000 \\
\hline $\mathrm{C}$ & 4.3627720000 & 1.8125560000 & -1.2923610000 \\
\hline $\mathrm{C}$ & 4.7610210000 & 0.6669790000 & -0.5944020000 \\
\hline C & 4.8440380000 & -3.8469910000 & 1.9409710000 \\
\hline $\mathrm{C}$ & 4.9905020000 & -2.3989020000 & 0.0087410000 \\
\hline $\mathrm{H}$ & 5.1127350000 & -4.7737380000 & 2.4507410000 \\
\hline $\mathrm{H}$ & 5.1307010000 & 2.4828200000 & -1.6817640000 \\
\hline & 5.3365090 & -3.5810990000 & 0.66086 \\
\hline & 5.3818580000 & -2.1918750000 & -0.9886710000 \\
\hline & 5.99 & -4.2985870000 & 0.1660860000 \\
\hline $\mathrm{H}$ & 6.076 & 0000 & 0000 \\
\hline $\mathrm{C}$ & 6.2157370000 & 0.4420870000 & -0.3944310000 \\
\hline H & 6.6950190000 & 0.7128260000 & -2.4810440000 \\
\hline $\mathrm{C}$ & 6.7480090000 & 0.1878390000 & 0.8802580000 \\
\hline $\mathrm{C}$ & 7.0978740000 & 0.5259020000 & -1.4834900000 \\
\hline $\mathrm{C}$ & 8.1197340000 & 0.0189760000 & 1.057 \\
\hline $\mathrm{C}$ & 8.4708 & 0.348 & -1.307 \\
\hline $\mathrm{H}$ & 8.5146450000 & -0.1729610000 & 2.0569100000 \\
\hline $\mathrm{C}$ & 8.9869900000 & 0.0941810000 & -0.0355200000 \\
\hline $\mathrm{H}$ & 9.1389570000 & 0.4063560000 & -2.1686130000 \\
\hline $\mathrm{H}$ & 10.0603400000 & -0.0448090000 & 0.1041940000 \\
\hline
\end{tabular}

\section{3-methylidene}

$\begin{array}{llll}\mathrm{H} & -6.9885800000 & 1.8679440000 & -1.8229440000 \\ \mathrm{H} & -6.5627410000 & 1.2964890000 & -3.4560440000 \\ \mathrm{C} & -6.1526970000 & 1.5837870000 & -2.4778900000\end{array}$ 


\begin{tabular}{|c|c|c|c|c|c|c|c|}
\hline $\mathrm{H}$ & -5.6697900000 & 1.0964790000 & 10000 & $\mathrm{C}$ & -0.3630490000 & 20000 & 100000 \\
\hline $\mathrm{H}$ & -5.5355700000 & 2.4841160000 & -2.6305760000 & $\mathrm{C}$ & -0.2853840000 & 2.1920020000 & 2.2448420000 \\
\hline C & -5.3323370000 & 0.4756550000 & -1.8752480000 & $\mathrm{H}$ & -0.2200140000 & 4.1882620000 & -1.3885850000 \\
\hline C & -5.1582900000 & 0.3822510000 & -0.4918110000 & $\mathrm{H}$ & -0.0167820000 & 5.5768120000 & -0.3190010000 \\
\hline $\mathrm{H}$ & -4.9986810000 & -0.0988680000 & 2.0802950000 & $\mathrm{C}$ & 0.0197850000 & 3.0557690000 & 1.1696210000 \\
\hline $\mathrm{H}$ & -4.8263260000 & -0.4236530000 & -3.7665020000 & $\mathrm{H}$ & 0.4169780000 & 0.9063250000 & 3.8242330000 \\
\hline $\mathrm{H}$ & -4.7302330000 & 2.6591910000 & 1.9318560000 & $\mathrm{H}$ & 0.5261130000 & -1.2053920000 & -2.4838420000 \\
\hline C & -4.6952970000 & -0.4709120000 & -2.6815770000 & $\mathrm{C}$ & 0.5329750000 & -2.3103610000 & 3.0318070000 \\
\hline C & -4.3445050000 & -0.5944880000 & 0.0880520000 & $\mathrm{C}$ & 0.5639230000 & -2.7181400000 & 1.6801760000 \\
\hline $\mathrm{H}$ & -4.3146320000 & 3.8870050000 & 3.1386000000 & $\mathrm{C}$ & 0.7742240000 & 1.3306960000 & 2.8775520000 \\
\hline $\mathrm{C}$ & -4.1809700000 & -0.6372740000 & 1.5834900000 & $\mathrm{H}$ & 0.9045580000 & -3.0332760000 & -0.9739590000 \\
\hline $\mathrm{H}$ & -4.1613570000 & -1.6694090000 & 1.9605160000 & $\mathrm{H}$ & 1.0562770000 & 0.4876420000 & 2.2294210000 \\
\hline $\mathrm{H}$ & -4.1156080000 & 2.1618100000 & 3.5250000000 & $\mathrm{O}$ & 1.1804690000 & 0.9318850000 & -0.3462840000 \\
\hline $\mathrm{H}$ & -4.0744050000 & -4.2093140000 & -0.6217960000 & $\mathrm{H}$ & 1.3770080000 & 1.4459350000 & -3.0927340000 \\
\hline $\mathrm{C}$ & -4.0138850000 & 2.9105720000 & 2.7285650000 & $\mathrm{C}$ & 1.4072110000 & 3.1137960000 & 0.6349230000 \\
\hline $\mathrm{C}$ & -3.8835730000 & -1.4755740000 & -2.1441950000 & $\mathrm{H}$ & 1.6915260000 & 1.9044480000 & 3.0749990000 \\
\hline $\mathrm{H}$ & -3.7639390000 & -3.4373490000 & -3.0613560000 & $\mathrm{H}$ & 1.7048560000 & -1.6979480000 & 4.7266120000 \\
\hline $\mathrm{C}$ & -3.6880980000 & -1.5251360000 & -0.7470310000 & $\mathrm{C}$ & 1.7325740000 & -2.0024860000 & 3.6762480000 \\
\hline $\mathrm{H}$ & -3.2330910000 & -0.1638220000 & 1.8835800000 & $\mathrm{C}$ & 1.7874790000 & -2.7714640000 & 0.9882490000 \\
\hline $\mathrm{C}$ & -3.2314350000 & -2.4733850000 & -3.0655560000 & $\mathrm{H}$ & 1.8425640000 & 5.0499730000 & 20000 \\
\hline $\mathrm{H}$ & -3.2295570000 & -2.1024960000 & -4.0997390000 & $\mathrm{C}$ & 1.8603860000 & -3.1896980000 & 20000 \\
\hline C & -3.1253460000 & -3.9121840000 & -0.1714290000 & $\mathrm{C}$ & 1.9278570000 & 2.0400830000 & -0.1174430000 \\
\hline $\mathrm{H}$ & -3.0640790000 & 4.4181890000 & 0.6740220000 & $\mathrm{H}$ & 2.0989810000 & -4.2620190000 & -0.5484100000 \\
\hline $\mathrm{C}$ & -2.7909640000 & -2.5522760000 & -0.1536040000 & $\mathrm{C}$ & 2.2339800000 & 4.2195520000 & 0.8658060000 \\
\hline $\mathrm{C}$ & -2.6090490000 & 2.9560130000 & 2.1920850000 & $\mathrm{C}$ & 2.4206560000 & 1.6061960000 & -3.4096700000 \\
\hline $\mathrm{H}$ & -2.5627990000 & -5.9267210000 & 0.3656540000 & $\mathrm{H}$ & 2.4584750000 & 1.4580050000 & -4.4971700000 \\
\hline $\mathrm{C}$ & -2.2818000000 & 3.8041890000 & 1.1283590000 & $\mathrm{H}$ & 2.6456510000 & -2.6287690000 & -0.9842760000 \\
\hline $\mathrm{C}$ & -2.2813270000 & -4.8728040000 & 0.3848680000 & $\mathrm{H}$ & 2.6715790000 & 2.6532010000 & -3.1895660000 \\
\hline $\mathrm{H}$ & -2.1953710000 & -2.6833840000 & -2.7650490000 & $\mathrm{C}$ & 2.9644850000 & -2.0651660000 & 3.0131200000 \\
\hline $\mathrm{O}$ & -1.8484330000 & 1.4919490000 & -1.2433610000 & $\mathrm{C}$ & 2.9677810000 & -2.4418450000 & 1.6691080000 \\
\hline $\mathrm{H}$ & -1.8303150000 & 1.4929010000 & 3.5694970000 & $\mathrm{C}$ & 3.2444020000 & 2.0602030000 & -0.6230660000 \\
\hline $\mathrm{H}$ & -1.6286400000 & 5.1910160000 & -0.9685750000 & $\mathrm{C}$ & 3.3488630000 & 0.6532010000 & -2.7056080000 \\
\hline $\mathrm{C}$ & -1.5983120000 & 2.1584060000 & 2. & $\mathrm{H}$ & 3.4949010000 & -0.6642570000 & -4.401 \\
\hline C & -1.5664820000 & -2.1763180000 & 60000 & $\mathrm{C}$ & 3.5350110000 & 4.2646670000 & 0.3641550000 \\
\hline $\mathrm{H}$ & -1.4061590000 & -1.4083390000 & 3.3123420000 & $\mathrm{C}$ & 3.7643080000 & 0.8885820000 & -1.3801370000 \\
\hline $\mathrm{H}$ & -1.3554380000 & -3.1246860000 & 3.7193460000 & $\mathrm{C}$ & 3.8158310000 & -0.4871940000 & -3.3707330000 \\
\hline $\mathrm{O}$ & -1.2156640000 & -0.8630140000 & 0.4989310000 & $\mathrm{H}$ & 3.9144530000 & -2.4843270000 & 1.1245590000 \\
\hline $\mathrm{C}$ & -1.0797860000 & -4.4787740000 & 0.9738400000 & $\mathrm{C}$ & 4.0321740000 & 3.1893150000 & -0.3727920000 \\
\hline $\mathrm{H}$ & -0.9953350000 & -0.5134140000 & -3.3016160000 & $\mathrm{H}$ & 4.1628580000 & 5.1370940000 & 0.5509630000 \\
\hline $\mathrm{C}$ & -0.9893780000 & 3.8685820000 & 0.6053620000 & $\mathrm{C}$ & 4.2393410000 & -1.7057940000 & 3.7292380000 \\
\hline $\mathrm{C}$ & -0.7761060000 & -2.1906760000 & 3.7647720000 & $\mathrm{H}$ & 4.2495700000 & -2.1075880000 & 4.7527440000 \\
\hline $\mathrm{C}$ & -0.7050920000 & -3.1305080000 & 1.0161830000 & $\mathrm{H}$ & 4.2607130000 & 0.3223530000 & 1.3475110000 \\
\hline $\mathrm{C}$ & -0.7008910000 & 4.7547560000 & -0.5770090000 & $\mathrm{H}$ & 4.3565510000 & -0.6127810000 & 3.8090810000 \\
\hline $\mathrm{H}$ & -0.6138870000 & -1.9326710000 & 4.8197320000 & $\mathrm{H}$ & 4.5161070000 & -3.5083650000 & -3.1800440000 \\
\hline Mo & -0.6102900000 & 0.4163080000 & -0.8118480000 & $\mathrm{C}$ & 4.6599170000 & -0.0008600000 & -0.7508710000 \\
\hline $\mathrm{H}$ & -0.4121450000 & -5.2196250000 & 1.4185280000 & $\mathrm{C}$ & 4.6834240000 & -1.3965660000 & -2.7592930000 \\
\hline
\end{tabular}




$\begin{array}{lrrr}\mathrm{H} & 5.0488160000 & 3.2162500000 & -0.7702190000 \\ \mathrm{H} & 5.0502860000 & -2.5344390000 & -4.5628460000 \\ \mathrm{C} & 5.1030490000 & -1.1270390000 & -1.4518000000 \\ \mathrm{C} & 5.1180750000 & 0.2374830000 & 0.6634520000 \\ \mathrm{H} & 5.1201210000 & -2.0930000000 & 3.1996110000 \\ \mathrm{C} & 5.1335660000 & -2.6432770000 & -3.4728830000 \\ \mathrm{H} & 5.6905660000 & 1.1721440000 & 0.7552530000 \\ \mathrm{H} & 5.7545110000 & -0.5861490000 & 1.0119810000 \\ \mathrm{H} & 5.7930930000 & -1.8178230000 & -0.9582870000 \\ \mathrm{H} & 6.1762300000 & -2.8925560000 & -3.2296490000\end{array}$

\section{3-TBP}

$\begin{array}{llll}\mathrm{H} & -7.1237440000 & -0.0569560000 & 2.9817010000\end{array}$

$\begin{array}{llll}\text { C } & -6.6914340000 & -0.3671670000 & 2.0181000000\end{array}$

$\mathrm{H} \quad-6.4343850000 \quad-1.4342750000 \quad 2.1117710000$

$\begin{array}{llll}\mathrm{H} & -6.2935020000 & 1.2162110000 & -0.1732440000\end{array}$

$\mathrm{H} \quad-6.2655540000 \quad-2.7615370000 \quad-0.3561230000$

$\mathrm{H} \quad-5.9088720000 \quad-2.8185040000 \quad-2.0992990000$

$\mathrm{H} \quad-5.7736720000 \quad-1.2877550000 \quad-1.1995170000$

$\begin{array}{llll}\text { C } & -5.6011420000 & -2.3743880000 & -1.1430990000\end{array}$

$\begin{array}{llll}\text { C } & -5.4829360000 & 0.4607460000 & 1.6720200000\end{array}$

$\begin{array}{llll}\text { C } & -5.4346480000 & 1.2218200000 & 0.5045520000\end{array}$

$\begin{array}{llll}\mathrm{H} & -4.9465790000 & 3.7915670000 & -0.8859050000\end{array}$

$\mathrm{H} \quad-4.8493660000 \quad 2.3338220000 \quad-1.8882870000$

$\begin{array}{llll}\mathrm{H} & -4.3795200000 & -0.1081960000 & 3.4382120000\end{array}$

$\begin{array}{llll}\text { C } & -4.3669320000 & 2.8705680000 & -1.0585700000\end{array}$

$\begin{array}{llll}\text { C } & -4.3647240000 & 0.4818080000 & 2.5168910000\end{array}$

$\begin{array}{llll}\text { C } & -4.3241750000 & 2.0173110000 & 0.1837470000\end{array}$

$\begin{array}{llll}\mathrm{H} & -4.2283730000 & -1.8423810000 & 1.1507840000\end{array}$

$\begin{array}{llll}\text { C } & -4.1573570000 & -2.6701310000 & -0.8387180000\end{array}$

$\mathrm{H} \quad-3.7442040000 \quad-3.5733490000 \quad-2.7509930000$

$\begin{array}{llll}\text { C } & -3.6016120000 & -2.3179410000 & 0.3952300000\end{array}$

$\mathrm{H} \quad-3.3637860000 \quad 3.1835550000 \quad-1.3739440000$

C $\quad-3.3281540000 \quad-3.2840230000 \quad-1.7814160000$

$\begin{array}{llll}\mathrm{H} & -3.2845140000 & 4.6015050000 & 1.5593320000\end{array}$

$\begin{array}{llll}\text { C } & -3.2300140000 & 1.2391780000 & 2.2223150000\end{array}$

$\begin{array}{llll}\text { C } & -3.2143010000 & 2.0263030000 & 1.0479370000\end{array}$

$\mathrm{H} \quad-2.8575680000 \quad 0.3545830000 \quad-1.2758720000$

$\mathrm{H} \quad-2.5196640000 \quad-1.8742720000 \quad 2.7196760000$

$\begin{array}{llll}\mathrm{H} & -2.5182780000 & -0.8781060000 & -2.5818730000\end{array}$

$\begin{array}{llll}\text { C } & -2.2977180000 & 4.3156490000 & 1.1888530000\end{array}$

$\begin{array}{llll}\text { C } & -2.2572010000 & -2.5517100000 & 0.6932840000\end{array}$

$\mathrm{H} \quad-2.2310520000 \quad 0.5904320000 \quad 4.0210000000$

$\begin{array}{llll}\text { C } & -2.1356420000 & -0.0491540000 & -1.9882500000\end{array}$

$\begin{array}{llll}\text { C } & -2.0870380000 & 2.9786020000 & 0.8338640000\end{array}$

$\begin{array}{llll}\text { C } & -2.0571610000 & 1.2541340000 & 3.1641860000\end{array}$

\begin{tabular}{|c|c|c|c|}
\hline & 5190000 & 0000 & -1.5 \\
\hline 1 & 000 & 2.006 & -2.6 \\
\hline & 1.8658420000 & 2.2694 & 3.5440030000 \\
\hline & -1.7148250000 & -2.2059500000 & 2.0527190000 \\
\hline & -1.6703260000 & -4.2158490000 & -3.5479840000 \\
\hline & 000 & 00 & 780000 \\
\hline & -1.4546020000 & 00 & 000 \\
\hline & -1.42 & -3.1 & -0.28 \\
\hline & -1.41 & 0 & -2 \\
\hline & -1.2777190000 & 5.2603060000 & 1.10 \\
\hline & -1 & -3. & 00 \\
\hline & -1.13 & 00 & 000 \\
\hline c & -1.1276150000 & -4.1 & 0000 \\
\hline $\mathrm{H}$ & -0.9680830000 & -1.4004660000 & 60000 \\
\hline $\mathrm{H}$ & -0.8487990000 & 100000 & -2.32 \\
\hline $\mathrm{C}$ & -0.81 & 00 & 000 \\
\hline $\mathrm{O}$ & -0.62 & 00 & 000 \\
\hline $\mathrm{H}$ & -0. & 0 & 0 \\
\hline & $-(-1$ & 0 & -1.2620280000 \\
\hline $\mathrm{H}$ & -0.1 & -3 . & -2 \\
\hline $\mathrm{C}$ & -0.0 & 4.8 & s \\
\hline $\mathrm{C}$ & -0.01 & -3.4 & 0 \\
\hline C & 0.12 & 1.3 & -2.6 \\
\hline $\mathrm{C}$ & 0.2378 & 3.535 & 0.26 \\
\hline $\mathrm{O}$ & 0.2613440000 & -1.2685430000 & -2.5294720000 \\
\hline $\mathrm{H}$ & 0.2809890000 & 4.5853 & 370000 \\
\hline $\mathrm{H}$ & 0.3022300000 & 2.3378930000 & -2.175 \\
\hline $\mathrm{C}$ & 0.3096710 & -4.8386810000 & 0.21 \\
\hline $\mathrm{O}$ & 0.7105940000 & -1.1776130000 & 00 \\
\hline 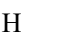 & 0 & 1 . & -3.4 \\
\hline & & & \\
\hline 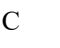 & 0.9862150000 & & 0.2536100000 \\
\hline 11 & 000 & S & 0 \\
\hline $\mathrm{H}$ & 1.2863450000 & .78407 & 80000 \\
\hline $\mathrm{C}$ & 1.3607130000 & .7886030000 & -2.172 \\
\hline $\mathrm{H}$ & 1.4869820000 & 5.8140520000 & -1.7900030000 \\
\hline $\mathrm{C}$ & 1.5969700000 & -5.2403030000 & 0.5561560000 \\
\hline$C$ & 1.6070 & 3.184 & -0.190 \\
\hline $\mathrm{H}$ & 000 & -1.34 & 0 \\
\hline 1 & 0 & 4 & -3 \\
\hline 11 & 02267700 & -6.29 & 0.6811180000 \\
\hline $\mathrm{C}$ & .8987850000 & 1.6753640000 & 1.8385 \\
\hline$c$ & 2.1409490000 & 3.7907150000 & -1.3509620000 \\
\hline $\mathrm{C}$ & 2.2951800000 & -2.9045160000 & 0.6048800000 \\
\hline $\mathrm{C}$ & 2.3986720000 & 2.2854800000 & 0.5570030000 \\
\hline $\mathrm{H}$ & 2.5192790000 & -2.8166450000 & 3.4380390000 \\
\hline
\end{tabular}




\begin{tabular}{lrrr}
$\mathrm{C}$ & 2.5767530000 & -4.2702940000 & 0.7467470000 \\
$\mathrm{C}$ & 2.6516300000 & -1.7393060000 & 3.2549860000 \\
$\mathrm{H}$ & 2.7411700000 & 1.3571950000 & 2.4656440000 \\
$\mathrm{H}$ & 2.9639530000 & -1.2650400000 & 4.1947010000 \\
$\mathrm{H}$ & 3.1290670000 & -1.8144930000 & -1.9385150000 \\
$\mathrm{C}$ & 3.4255160000 & -1.9649210000 & 0.8573220000 \\
$\mathrm{C}$ & 3.4311610000 & 3.4463790000 & -1.7666980000 \\
$\mathrm{H}$ & 3.5943360000 & -4.5584730000 & 1.0192300000 \\
$\mathrm{C}$ & 3.6565520000 & -1.4884800000 & 2.1628760000 \\
$\mathrm{C}$ & 3.6869720000 & 1.9793150000 & 0.1098340000 \\
$\mathrm{H}$ & 3.8308910000 & 3.9045720000 & -2.6762170000 \\
$\mathrm{H}$ & 4.0689010000 & -3.2749980000 & -1.6365690000 \\
$\mathrm{C}$ & 4.1020210000 & -2.1765070000 & -1.5725880000 \\
$\mathrm{C}$ & 4.2183630000 & 2.5366930000 & -1.0570860000 \\
$\mathrm{H}$ & 4.2872690000 & 1.2800770000 & 0.6931110000 \\
$\mathrm{C}$ & 4.3611540000 & -1.7118470000 & -0.1643520000 \\
$\mathrm{C}$ & 4.8441330000 & -0.8007910000 & 2.4352620000 \\
$\mathrm{H}$ & 4.8845010000 & -1.8170130000 & -2.2541210000 \\
$\mathrm{H}$ & 5.0238060000 & -0.4391070000 & 3.4520030000 \\
$\mathrm{C}$ & 5.5448990000 & -1.0360060000 & 0.1535080000 \\
$\mathrm{H}$ & 5.5541330000 & 1.2189420000 & -2.1240720000 \\
$\mathrm{C}$ & 5.5899660000 & 2.1571450000 & -1.5471630000 \\
$\mathrm{C}$ & 5.8115520000 & -0.5808690000 & 1.4481540000 \\
$\mathrm{H}$ & 6.0097690000 & 2.9331320000 & -2.2022970000 \\
$\mathrm{H}$ & 6.2791010000 & -0.8582150000 & -0.6367970000 \\
$\mathrm{H}$ & 6.2853360000 & 1.9937440000 & -0.7122770000 \\
$\mathrm{H}$ & 6.8987200000 & 1.2637460000 & 1.7452420000 \\
$\mathrm{H}$ & 7.0753520000 & 0.1751030000 & 1.7604820000 \\
\hline & 7.4576910000 & -0.0749930000 & 2.7603880000 \\
\hline
\end{tabular}

\section{3-SP}

$\mathrm{H} \quad-7.7608820000$

$\mathrm{H} \quad-7.2650030000$

C $\quad-6.9354920000$

$\mathrm{H} \quad-6.7590800000$

H $\quad-6.2820870000$

H $\quad-6.2727070000$

H $\quad-5.9676970000$

C $\quad-5.6898220000$

C $\quad-5.5526080000$

C $\quad-5.4942420000$

$\mathrm{H} \quad-5.4549610000$

$\mathrm{H} \quad-4.9731460000$

$\mathrm{H} \quad-4.7949780000$

C $\quad-4.6690980000$

C $\quad-4.3307010000$
$-0.1179470000$

$-0.0142980000$

0.1525260000

1.2355260000

1.8550410000

$-1.0917870000$

2.6707380000

$-0.6313960000$

1.9726280000

$-1.1989980000$

0.9890630000

$-2.2022390000$

$-0.3225690000$

$-0.7683480000$

$-1.9083110000$
1.1501600000

2.8581390000

1.8227420000

1.7104700000

$-0.8374350000$

$-0.5150490000$

$-2.3910640000$

1.5075540000

$-1.6511490000$

0.2452640000

$-2.1382940000$

$-2.1132850000$

3.4461120000

2.4550260000

$-0.0746180000$$$
\text { C }
$$$$
\text { C }
$$

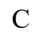$$
\text { . }
$$

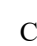$$
\text { (n) }
$$

$$
\text { t. }
$$$$
\begin{array}{llll}
\text { C } & -2.4294000000 & -1.6252660000 & 3.2303200000
\end{array}
$$$$
\begin{array}{llll}
\mathrm{H} & -2.2932470000 & -2.6810700000 & 3.5106450000
\end{array}
$$$$
\begin{array}{llll}
\text { C } & -2.2011290000 & -3.0076920000 & 0.6803500000
\end{array}
$$$$
\begin{array}{llll}
\text { C } & -2.1926310000 & 3.7839720000 & -1.4193870000
\end{array}
$$$$
\begin{array}{llll}
\text { C } & -1.9708050000 & 1.8306500000 & 1.8756450000
\end{array}
$$$$
\begin{array}{llll}
\mathrm{H} & -1.7739150000 & 4.7216080000 & -3.3112650000
\end{array}
$$$$
\begin{array}{llll}
\mathrm{H} & -1.6770290000 & -6.3748600000 & 1.0426640000
\end{array}
$$$$
\begin{array}{llll}
\text { C } & -1.6754250000 & 3.2585330000 & -0.2129570000
\end{array}
$$$$
\begin{array}{llll}
\mathrm{H} & -1.5137040000 & -0.9374770000 & -2.6149530000
\end{array}
$$$$
\mathrm{H} \quad-1.5118350000 \quad 5.7856390000 \quad-1.9181320000
$$$$
\begin{array}{llll}
\text { C } & -1.4595420000 & -5.3288680000 & 0.8220980000
\end{array}
$$$$
\mathrm{H} \quad-1.4535230000 \quad-1.2694680000 \quad 2.8672110000
$$$$
\begin{array}{llll}
\text { C } & -1.4109120000 & 4.7487260000 & -2.2750210000
\end{array}
$$$$
\begin{array}{llll}
\mathrm{H} & -1.3727480000 & 2.5763440000 & 2.4176050000
\end{array}
$$$$
\mathrm{H}
$$

.

$$
\text { (1) }
$$

$$
\mathrm{H}
$$$$
\text { H. }
$$

\section{c}

$$
c
$$




$\begin{array}{llll}\mathrm{O} & 0.5525670000 & 1.4023010000 & 0.1619910000 \\ \mathrm{H} & 0.5634010000 & 2.2122590000 & -2.5014540000 \\ \mathrm{H} & 0.6159880000 & -5.6793080000 & 0.3313190000 \\ \mathrm{C} & 0.7361780000 & 2.7212880000 & 0.3786760000 \\ \mathrm{H} & 0.8642490000 & 0.3083050000 & -4.1292200000 \\ \mathrm{H} & 1.0351730000 & -1.4475070000 & 1.9997690000 \\ \mathrm{H} & 1.1493820000 & -5.4339840000 & -2.3147350000 \\ \mathrm{C} & 1.1507730000 & -4.3499310000 & -2.5116940000 \\ \mathrm{C} & 1.1958150000 & 5.4186350000 & 0.9870920000 \\ \mathrm{H} & 1.2258770000 & 1.2256700000 & 2.9079200000 \\ \mathrm{H} & 1.3702780000 & 6.4675300000 & 1.2316990000 \\ \mathrm{H} & 1.4024920000 & -3.0758190000 & 2.5737810000 \\ \mathrm{C} & 1.4985420000 & -3.2363120000 & -0.2487900000 \\ \mathrm{H} & 1.5320860000 & -4.2007510000 & -3.5310190000 \\ \mathrm{C} & 1.8314510000 & -2.2066130000 & 2.0530590000 \\ \mathrm{C}\end{array}$

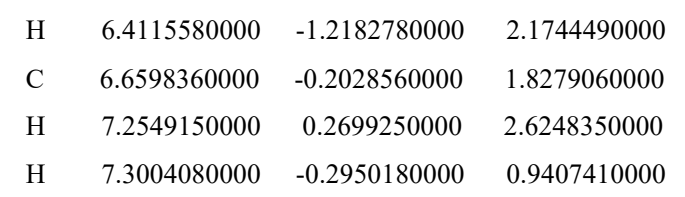

\section{1@SiO $\mathrm{SiO}_{2}$-methylidene}

\begin{tabular}{|c|c|c|c|}
\hline $\mathrm{F}$ & -4.3611480000 & -0.4843840000 & -0.5083160000 \\
\hline $\mathrm{F}$ & -3.6731630000 & 1.9665130000 & 0.6155030000 \\
\hline $\mathrm{F}$ & -3.3377740000 & 0.4278260000 & 2.1322290000 \\
\hline $\mathrm{F}$ & -3.1645990000 & -1.8644280000 & 0.6905760000 \\
\hline $\mathrm{C}$ & -3.1156250000 & -0.9520050000 & -0.2957790000 \\
\hline $\mathrm{F}$ & -2.8028620000 & 1.3181730000 & -1.9718460000 \\
\hline $\mathrm{C}$ & -2.7471420000 & 1.1490600000 & 1.1590360000 \\
\hline $\mathrm{F}$ & -2.7047640000 & -1.5871150000 & -1.4177660000 \\
\hline $\mathrm{C}$ & -2.1016190000 & 0.1980250000 & 0.0774980000 \\
\hline $\mathrm{F}$ & -1.7984050000 & 1.9126310000 & 1.7348970000 \\
\hline $\mathrm{C}$ & -1.7353500000 & 1.0317010000 & -1.2094460000 \\
\hline $\mathrm{F}$ & -1.1231480000 & 2.1846020000 & -0.8761040000 \\
\hline $\mathrm{O}$ & -0.9673570000 & -0.3375640000 & 0.6430100000 \\
\hline $\mathrm{F}$ & -0.8565100000 & 0.3217290000 & -1.9702090000 \\
\hline $\mathrm{O}$ & 0.3674990000 & -2.5922190000 & -1.1502960000 \\
\hline Mo & 0.5793840000 & -1.3531120000 & -0.0194630000 \\
\hline $\mathrm{H}$ & 0.8963330000 & 1.6396510000 & 1.6528230000 \\
\hline $\mathrm{C}$ & 1.1660490000 & -2.2746460000 & 1.5162800000 \\
\hline $\mathrm{H}$ & 1.3310590000 & -3.3473190000 & 1.6392080000 \\
\hline $\mathrm{H}$ & 1.4168540000 & -1.6404970000 & 2.3800450000 \\
\hline $\mathrm{H}$ & 1.6905270000 & 3.1425740000 & 2.1920360000 \\
\hline $\mathrm{C}$ & 1.8638760000 & 2.0917380000 & 1.9256940000 \\
\hline $\mathrm{O}$ & 1.9337880000 & -0.1179500000 & -0.4872990000 \\
\hline $\mathrm{H}$ & 2.2776080000 & 1.5666820000 & 2.8015540000 \\
\hline $\mathrm{O}$ & 2.7814750000 & 2.0643420000 & 0.8314070000 \\
\hline $\mathrm{H}$ & 2.9458820000 & 1.1991770000 & -2.7991190000 \\
\hline $\mathrm{Si}$ & 3.2728080000 & 0.6907320000 & 0.0949380000 \\
\hline $\mathrm{H}$ & 3.5080810000 & 2.7603490000 & -2.1149380000 \\
\hline $\mathrm{C}$ & 3.8003880000 & 1.7234370000 & -2.3424960000 \\
\hline $\mathrm{H}$ & 4.0091580000 & -2.2265630000 & 0.5180910000 \\
\hline $\mathrm{O}$ & 4.0842800000 & -0.2365520000 & 1.1700540000 \\
\hline $\mathrm{O}$ & 4.2479200000 & 1.0510050000 & -1.1622620000 \\
\hline $\mathrm{H}$ & 4.6346220000 & 1.7353990000 & -3.0560150000 \\
\hline $\mathrm{C}$ & 4.7394770000 & -1.4602500000 & 0.8243590000 \\
\hline $\mathrm{H}$ & 5.2729100000 & -1.8157410000 & 1.7158450000 \\
\hline $\mathrm{H}$ & 5.4637210000 & -1.3040270000 & 0.0103320000 \\
\hline
\end{tabular}

\section{1@SiO SBP-axial $_{2}$}

$\begin{array}{llll}\text { F } & -4.4678780000 & 0.0594090000 & 0.5789470000 \\ \text { F } & -3.5247740000 & 1.5703710000 & -0.6849570000 \\ \text { F } & -3.4968240000 & -2.2953370000 & -0.5398870000 \\ \text { F } & -3.4463730000 & -0.7577170000 & -2.0933450000 \\ \text { C } & -3.3058170000 & 0.6912990000 & 0.3051840000 \\ \text { F } & -2.9428870000 & 1.3912220000 & 1.4053790000 \\ \text { C } & -2.7164770000 & -1.3697630000 & -1.1391670000 \\ \text { F } & -2.6112350000 & -1.4800760000 & 1.9877390000 \\ \text { C } & -2.1570050000 & -0.3153290000 & -0.1061940000 \\ \text { F } & -1.7014240000 & -2.0105320000 & -1.7498520000 \\ \text { C } & -1.6239290000 & -1.0657100000 & 1.1734830000 \\ \text { O } & -1.1450570000 & 0.3596460000 & -0.7309430000\end{array}$




\begin{tabular}{lccc}
$\mathrm{F}$ & -0.8747520000 & -2.1335660000 & 0.8367130000 \\
$\mathrm{~F}$ & -0.8232170000 & -0.2275780000 & 1.8891820000 \\
$\mathrm{O}$ & -0.5352400000 & 3.0133110000 & -0.0445040000 \\
$\mathrm{Mo}$ & 0.3407350000 & 1.5438740000 & -0.2487360000 \\
$\mathrm{H}$ & 1.0704650000 & -2.3191910000 & -1.7263350000 \\
$\mathrm{H}$ & 1.2163490000 & 2.9815650000 & 1.9101130000 \\
$\mathrm{H}$ & 1.4040240000 & 3.3851880000 & -1.9699340000 \\
$\mathrm{O}$ & 1.5524870000 & -0.0282570000 & -0.2693140000 \\
$\mathrm{C}$ & 1.6012230000 & 2.1892720000 & 1.2681510000 \\
$\mathrm{H}$ & 1.6448930000 & -1.2793540000 & 2.8056370000 \\
$\mathrm{C}$ & 1.7135350000 & 2.4723840000 & -1.4611730000 \\
$\mathrm{H}$ & 1.9138280000 & -3.8968750000 & -1.6517670000 \\
$\mathrm{C}$ & 2.0553230000 & -2.8079130000 & -1.6734100000 \\
$\mathrm{H}$ & 2.1779810000 & 1.4201080000 & 1.7843260000 \\
$\mathrm{H}$ & 2.3323900000 & 1.7991340000 & -2.0570110000 \\
$\mathrm{C}$ & 2.3470720000 & 2.7460850000 & -0.0201290000 \\
$\mathrm{H}$ & 2.3878170000 & 3.8328550000 & 0.0903530000 \\
$\mathrm{H}$ & 2.4686320000 & -2.7495100000 & 2.1963080000 \\
$\mathrm{C}$ & 2.6243790000 & -1.7248900000 & 2.5673940000 \\
$\mathrm{H}$ & 2.6350610000 & -2.5445050000 & -2.5733100000 \\
$\mathrm{O}$ & 2.7568950000 & -2.4342680000 & -0.4852970000 \\
$\mathrm{Si}$ & 2.9127610000 & -0.8769130000 & 0.0173630000 \\
$\mathrm{H}$ & 3.2296790000 & -1.7613840000 & 3.4831970000 \\
$\mathrm{O}$ & 3.3267270000 & -0.9290750000 & 1.6091670000 \\
$\mathrm{H}$ & 3.3269010000 & 2.2591190000 & -0.0419690000 \\
$\mathrm{O}$ & 4.1175550000 & -0.0528450000 & -0.7553510000 \\
$\mathrm{H}$ & 5.4681250000 & -0.5238100000 & -0.7521860000 \\
$\mathrm{H}$ & 5.5311540000 & -1.5578850000 & -1.1279120000 \\
$\mathrm{H}$ & 5.8948430000 & -0.4884920000 & 0.2626540000 \\
\hline & 6.0552270000 & 0.1297090000 & -1.4115600000
\end{tabular}

\section{1@SiO2-TBP-equatorial}

$\begin{array}{lrrr}\text { F } & -4.0504690000 & -0.6918290000 & 1.2234190000 \\ \text { F } & -3.7779510000 & -1.0814620000 & -1.5232540000 \\ \text { F } & -3.4770220000 & 1.0616040000 & -1.2057310000 \\ \text { F } & -2.9985890000 & 1.2078290000 & 1.4369080000 \\ \text { C } & -2.8435940000 & -0.1345640000 & -1.3129820000 \\ \text { C } & -2.8290470000 & -0.1263990000 & 1.2672680000 \\ \text { F } & -2.4943960000 & -2.7723280000 & 0.2908850000 \\ \text { F } & -2.1892650000 & -0.5841470000 & 2.3664760000 \\ \text { F } & -2.0578480000 & -0.0674220000 & -2.4066100000 \\ \text { C } & -1.9681360000 & -0.4070630000 & -0.0269620000 \\ \text { H } & -1.7579890000 & 3.1645120000 & 0.3191930000 \\ \text { C } & -1.4946580000 & -1.9181850000 & -0.0218060000 \\ \text { H } & -1.2150790000 & 2.3836850000 & -1.8017430000 \\ \text { F } & -1.0160630000 & -2.2650050000 & -1.2369200000 \\ \text { O } & -0.8849130000 & 0.4002300000 & -0.0392960000\end{array}$

$\begin{array}{lccc}\mathrm{H} & -0.7024340000 & 2.0471860000 & 2.0481530000 \\ \mathrm{C} & -0.6826460000 & 3.3043770000 & 0.1825710000 \\ \mathrm{~F} & -0.5042570000 & -2.0897590000 & 0.8719370000 \\ \mathrm{H} & -0.3728770000 & 4.3515030000 & 0.2371460000 \\ \mathrm{C} & -0.3518990000 & 2.8144870000 & -1.2941470000 \\ \mathrm{C} & 0.0167890000 & 2.5705170000 & 1.4177990000 \\ \mathrm{H} & 0.1807190000 & 3.5627620000 & -1.8812550000 \\ \mathrm{H} & 0.6962930000 & 3.2236410000 & 1.9654440000 \\ \mathrm{Mo} & 0.8061990000 & 1.5073010000 & -0.1758400000 \\ \mathrm{H} & 1.2759730000 & -0.3284120000 & 2.4857350000 \\ \mathrm{H} & 1.5550810000 & -2.6708810000 & -1.8789590000 \\ \mathrm{O} & 1.7474820000 & -0.1078480000 & -0.5082080000 \\ \mathrm{H} & 1.8685050000 & -2.0071590000 & 2.6723440000 \\ \mathrm{O} & 2.1226970000 & 2.6001960000 & -0.2534430000 \\ \mathrm{H} & 2.1337310000 & -4.2284720000 & -1.2184320000 \\ \mathrm{C} & 2.1709000000 & -0.9486010000 & 2.6714030000 \\ \mathrm{C} & 2.4000020000 & -3.1763030000 & -1.3857990000 \\ \mathrm{H} & 2.5769790000 & -0.6882110000 & 3.6579450000 \\ \mathrm{O} & 2.6807040000 & -2.5913280000 & -0.1131260000 \\ \mathrm{Si} & 3.0070240000 & -1.0052290000 & 0.0999250000 \\ \mathrm{O} & 3.1876990000 & -0.7025750000 & 1.7019070000 \\ \mathrm{H} & 3.2836600000 & -3.1301720000 & -2.0416740000 \\ \mathrm{H} & 4.3091630000 & 1.3457350000 & -1.3367780000 \\ \mathrm{O} & 4.3999370000 & -0.6403500000 & -0.6721670000 \\ \mathrm{C} & 4.9559130000 & 0.6745730000 & -0.7513990000 \\ \mathrm{H} & 5.0992430000 & 1.1068480000 & 0.2514160000 \\ \mathrm{H} & 5.9324610000 & 0.5930190000 & -1.2471900000 \\ \mathrm{H} & \mathrm{Si} 7 \mathrm{SP} & & \\ \mathrm{H}\end{array}$

\section{1@SiO 2 -SP}

$\begin{array}{lrrr}\text { F } & -4.3759360000 & 0.1680830000 & 0.1403530000 \\ \text { F } & -3.3281550000 & -2.1809780000 & -0.9558320000 \\ \text { C } & -3.1524290000 & 0.7311360000 & 0.1160950000 \\ \text { F } & -3.1328900000 & 1.6571750000 & -0.8606970000 \\ \text { F } & -2.9700030000 & -1.5679250000 & 1.7592020000 \\ \text { F } & -2.9585060000 & 1.3771430000 & 1.2896560000 \\ \text { F } & -2.8528120000 & -0.5689950000 & -2.3543460000 \\ \text { C } & -2.3831200000 & -1.2827660000 & -1.3100790000 \\ \text { C } & -2.0063840000 & -0.3285480000 & -0.1096280000 \\ \text { C } & -1.8066530000 & -1.1874800000 & 1.2011870000 \\ \text { F } & -1.2973660000 & -1.9550960000 & -1.7294500000 \\ \text { F } & -1.1218720000 & -0.4601490000 & 2.1220050000 \\ \text { F } & -1.0757330000 & -2.2898930000 & 0.9458130000 \\ \text { H } & -0.8958680000 & 3.2088800000 & -1.0588710000 \\ \text { O } & -0.8413810000 & 0.3066410000 & -0.4614260000 \\ \text { C } & 0.0985870000 & 2.7647280000 & -1.2079530000 \\ \text { H } & 0.1055490000 & 2.1456840000 & -2.1158780000 \\ \text { O } & 0.1862310000 & 2.4155800000 & 1.7354120000\end{array}$




$\begin{array}{lccc}\mathrm{Mo} & 0.5352240000 & 1.3928200000 & 0.4350050000 \\ \mathrm{H} & 1.1625320000 & 4.4415020000 & -0.3395930000 \\ \mathrm{C} & 1.3366100000 & 3.6295030000 & -1.0599420000 \\ \mathrm{H} & 1.6191040000 & -2.8572300000 & 2.1754500000 \\ \mathrm{H} & 1.7323020000 & 4.0750550000 & -1.9888410000 \\ \mathrm{O} & 1.8689860000 & 0.0872930000 & 0.8367360000 \\ \mathrm{H} & 2.0528670000 & -4.3051170000 & 1.2198880000 \\ \mathrm{C} & 2.1823840000 & 2.5304680000 & -0.4425150000 \\ \mathrm{O} & 2.1933800000 & -2.5364600000 & 0.1929650000 \\ \mathrm{H} & 2.2095420000 & -2.4200520000 & -2.6353420000 \\ \mathrm{C} & 2.3140160000 & -3.2578200000 & 1.4209720000 \\ \mathrm{O} & 2.4737500000 & -0.6144480000 & -1.6131940000 \\ \mathrm{H} & 2.4825600000 & -0.9586200000 & -3.6347900000 \\ \mathrm{H} & 2.6239000000 & 1.8598050000 & -1.1927010000 \\ \mathrm{Si} & 2.7384760000 & -1.0163220000 & -0.0428250000 \\ \mathrm{C} & 2.7742590000 & -1.4791560000 & -2.7133530000 \\ \mathrm{H} & 2.9154790000 & 2.7776280000 & 0.3382980000 \\ \mathrm{H} & 3.3422130000 & -3.2105640000 & 1.8116760000 \\ \mathrm{H} & 3.8512510000 & -1.7072780000 & -2.7566130000 \\ \mathrm{O} & 4.3132620000 & -0.9801550000 & 0.4163350000 \\ \mathrm{H} & 4.6155210000 & 0.9014550000 & 1.2854530000 \\ \mathrm{C} & 5.0740090000 & 0.2208470000 & 0.5504430000 \\ \mathrm{H} & 5.1649460000 & 0.7468920000 & -0.4138150000 \\ \mathrm{H} & 6.0780180000 & -0.0553450000 & 0.8986750000\end{array}$

\section{2@SiO -methylidene $^{-}$}

$\begin{array}{lrrr}\mathrm{H} & -5.7474990000 & 0.5884840000 & -2.1650360000 \\ \mathrm{H} & -5.3337970000 & -0.7952440000 & 2.2727420000 \\ \mathrm{H} & -5.1520630000 & 1.3058360000 & 0.1487420000 \\ \mathrm{H} & -5.0521890000 & -1.8181920000 & 0.8326010000 \\ \mathrm{H} & -4.8030890000 & -2.4919590000 & 2.4806950000 \\ \mathrm{C} & -4.7048760000 & -1.5923070000 & 1.8529540000 \\ \mathrm{C} & -4.7038930000 & 0.7554220000 & -1.8925090000 \\ \mathrm{C} & -4.3707710000 & 1.1609290000 & -0.5993500000 \\ \mathrm{H} & -3.9418590000 & 0.2441740000 & -3.8503690000 \\ \mathrm{C} & -3.6914810000 & 0.5611860000 & -2.8362550000 \\ \mathrm{O} & -3.3585870000 & -1.1196880000 & 1.8276730000 \\ \mathrm{H} & -3.3300920000 & 6.9452360000 & 0.4194370000 \\ \mathrm{H} & -3.2666860000 & 5.7715220000 & -1.7796190000 \\ \mathrm{C} & -3.0379790000 & 1.3775000000 & -0.2526690000 \\ \mathrm{H} & -2.7842150000 & 1.6866930000 & 0.7613580000 \\ \mathrm{C} & -2.6906130000 & 6.0717140000 & 0.2819510000 \\ \mathrm{C} & -2.6572440000 & 5.4111230000 & -0.9490000000 \\ \mathrm{C} & -2.3587390000 & 0.7729970000 & -2.4873280000 \\ \mathrm{O} & -2.0943650000 & -3.2108900000 & 2.8013820000 \\ \mathrm{Si} & -2.0521670000 & -2.0746940000 & 1.6157030000 \\ \mathrm{C} & -2.0138130000 & 1.1915730000 & -1.1917540000\end{array}$

\begin{tabular}{|c|c|c|c|}
\hline & 000 & 00 & \\
\hline & 0 & 0 & 2.3008970000 \\
\hline & .8994490000 & 5.6072720000 & 10000 \\
\hline & -1.8475890000 & 4.2907630000 & -1.1247400000 \\
\hline & -1.8285570000 & 3.7782590000 & -2.0870730000 \\
\hline & -1.5700170000 & 0.6202030000 & -3.2254400000 \\
\hline & -1.4197320000 & -4.9925360000 & 1.9295770000 \\
\hline & -1.4073070000 & -4.9129530000 & 3.7195690000 \\
\hline c & -1.2207850000 & -4.3456480000 & 2.7979940000 \\
\hline & -1.1973720000 & 550000 & 630000 \\
\hline & -1.0864200000 & -3.2975500000 & -3.0750250000 \\
\hline $\mathrm{C}$ & -1.0829150000 & 4.4900810000 & 1.1553670000 \\
\hline c & -1.0485080000 & 3.8118970000 & -0.0737170000 \\
\hline C & -0.7034530000 & -1.1214890000 & 1.6385350000 \\
\hline C & -0.5959130000 & 1.4286190000 & -0.8197950000 \\
\hline $\mathrm{H}$ & -0.4745370000 & 4.1202600000 & 1.9834780000 \\
\hline $\mathrm{C}$ & -0.3789320000 & -3.6691860000 & -2.3189050000 \\
\hline $\mathrm{C}$ & -0.3625260000 & -0.1215300000 & 2.5973180000 \\
\hline Mo & -0.2109960000 & -2.6056150000 & -0.7666390000 \\
\hline $\mathrm{H}$ & -0.1630370000 & -4.0387320000 & 90000 \\
\hline $\mathrm{C}$ & 700000 & 0000 & 40000 \\
\hline 0 & -0.1299740000 & -0.8005090000 & 3000 \\
\hline & -0.10 & 0000 & 20000 \\
\hline & 0.1334470000 & -4.60 & 70000 \\
\hline $\mathrm{C}$ & 0.3396160000 & 0.3781280000 & -0.9677 \\
\hline $\mathrm{H}$ & 0.5044790000 & 0.4305760000 & 2.2105770000 \\
\hline $\mathrm{O}$ & 0.9868260000 & -3.4172420000 & 0.1207040000 \\
\hline $\mathrm{C}$ & 1.1861500000 & 2.7636430000 & 0.1418510000 \\
\hline $\mathrm{H}$ & 1.5325570000 & 3.7243010000 & 0.5256820000 \\
\hline $\mathrm{C}$ & 1.6953170000 & 0.5063350000 & -0.5880520000 \\
\hline $\mathrm{C}$ & 2.1181860000 & 1.7334560000 & -0.0280850000 \\
\hline $\mathrm{H}$ & 2.20 & -0.9 & 0 \\
\hline $\mathrm{C}$ & 2. & -0 & 00 \\
\hline $\mathrm{C}$ & 000 & -1 & 00 \\
\hline $\mathrm{H}$ & 410000 & 70000 & 0000 \\
\hline $\mathrm{H}$ & 3.1949770000 & .0000 & 70000 \\
\hline $\mathrm{C}$ & 3.3093910000 & -1.1356260000 & 0.4041090000 \\
\hline $\mathrm{C}$ & 3.5294740000 & 1.9900490000 & 0.3534740000 \\
\hline $\mathrm{C}$ & 3.5927370000 & -2.4429140000 & -2.0427360000 \\
\hline $\mathrm{H}$ & 3.6987780000 & -2.9501480000 & -3.0031860000 \\
\hline $\mathrm{C}$ & 3.8293 & 2.559 & 1.6013940000 \\
\hline c & 100000 & 550000 & 2976240000 \\
\hline $\mathrm{C}$ & 4.2936570000 & -2.8996330000 & -0.9261860000 \\
\hline $\mathrm{H}$ & 4.3725900000 & 1.2737310000 & -1.5011880000 \\
\hline $\mathrm{C}$ & 4.5887980000 & 1.7094970000 & -0.5251290000 \\
\hline & 4.6833000000 & -2.6002500000 & 1.1780810000 \\
\hline
\end{tabular}




$\begin{array}{llll}\mathrm{H} & 4.9479720000 & -3.7690440000 & -1.0069730000 \\ \mathrm{C} & 5.1484770000 & 2.8309030000 & 1.9666190000 \\ \mathrm{H} & 5.3614400000 & 3.2636980000 & 2.9458090000 \\ \mathrm{C} & 5.9056790000 & 1.9866850000 & -0.1639040000 \\ \mathrm{C} & 6.1922710000 & 2.5441140000 & 1.0850240000 \\ \mathrm{H} & 6.7143540000 & 1.7668210000 & -0.8631350000 \\ \mathrm{H} & 7.2246840000 & 2.7549350000 & 1.3687330000\end{array}$

\section{2@ $\mathrm{SiO}_{2}$-TBP}

\begin{tabular}{|c|c|c|c|}
\hline $\mathrm{H}$ & -6.5 & 00 & 3.1767760000 \\
\hline $\mathrm{H}$ & -5.9084110000 & 1.4828070000 & 3.2664810000 \\
\hline$C$ & -5.6646580000 & .4171570000 & 30000 \\
\hline 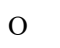 & -5.0785180000 & 00 & 0 \\
\hline $\mathrm{H}$ & -4. & 0 & 0 \\
\hline $\mathrm{H}$ & -4.81 & 000 & 0000 \\
\hline $\mathrm{H}$ & -3.9729920000 & -1.57138 & 20000 \\
\hline C & -3.792 & 950000 & 0000 \\
\hline $\mathrm{H}$ & -3.7290790000 & 0.7315310000 & -3.9411950000 \\
\hline $\mathrm{H}$ & -3.6364990000 & 2.2050480000 & -1.3650520000 \\
\hline $\mathrm{O}$ & -3.5843090000 & -2.3918510000 & 1.4713840000 \\
\hline $\mathrm{Si}$ & -3.57 & -0.74 & 0000 \\
\hline C & -3.2 & 000 & 000 \\
\hline $\mathrm{O}$ & -3.2 & -0 & -0 \\
\hline C & -3.0 & 00 & 00 \\
\hline $\mathrm{H}$ & -2.9192760000 & -4.25 & 0000 \\
\hline $\mathrm{H}$ & -2.8739480000 & -1.4078450000 & -3.5567350000 \\
\hline $\mathrm{H}$ & -2.7956270000 & -3.0379540000 & -0.3595860000 \\
\hline $\mathrm{H}$ & -2.7659110000 & 3.4675970000 & 1.5513510000 \\
\hline $\mathrm{C}$ & -2.6833780000 & -3.2072470000 & 0.7235890000 \\
\hline 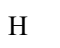 & -2.5888760000 & 5.2484570000 & -0.1920710000 \\
\hline $\mathrm{H}$ & -2.561 & 2.428 & -2.827 \\
\hline $\mathrm{O}$ & -2 & -0.1 & 00 \\
\hline C & 0 & 3 & 0 \\
\hline U & 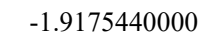 & 0 & 00 \\
\hline C & -1.84 & 0 & 0000 \\
\hline $\mathrm{H}$ & -1.6693650000 & -1.9364770000 & 3.3369610000 \\
\hline $\mathrm{H}$ & -1.6318030000 & -3.0196610000 & 0.9895960000 \\
\hline $\mathrm{C}$ & -1.4182440000 & -0.8855020000 & 3.1216040000 \\
\hline $\mathrm{H}$ & -1.0955570000 & -0.4019090000 & 4.0546400000 \\
\hline $\mathrm{H}$ & -1.0898450000 & 1.6626510000 & 1.6581760000 \\
\hline$C$ & -0.9925160000 & 2.4386100000 & 0.8986160000 \\
\hline $\mathrm{C}$ & -0.794 & 4.412 & -1.06332 \\
\hline 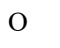 & -0.7829490000 & 0.1997950000 & -2.7832360000 \\
\hline $\mathrm{H}$ & -0.7143090000 & 5.1800450000 & -1.8355090000 \\
\hline $\mathrm{H}$ & -0.5853930000 & -0.8605570000 & 2.4008570000 \\
\hline $\mathrm{O}$ & -0.5754660000 & -0.3404230000 & -0.1469460000 \\
\hline & -0.4850220000 & -4.5097790000 & -2.3683640000 \\
\hline
\end{tabular}

\begin{tabular}{|c|c|c|c|}
\hline & -0.1205870000 & -6.1323410000 & -0.50978300 \\
\hline & 0.0387510000 & -4.1970430000 & -1.4628930000 \\
\hline & 0.0794070000 & 2.4015880000 & -0.0108720000 \\
\hline & 0.1585670000 & 3.3975170000 & -0.9984050000 \\
\hline & 0.2441740000 & -5.1074460000 & -0.4228710000 \\
\hline & 0.3485870000 & -2.1773000000 & -2.1668870000 \\
\hline & 0.5023610000 & -2.8863760000 & 50000 \\
\hline & 0.7334900000 & -0.0152730000 & -0.0756090000 \\
\hline & 0.9203 & -4 & 00 \\
\hline & 0.9805 & 000 & 0000 \\
\hline & 1.0850580000 & -5.4011460000 & 1.5463370000 \\
\hline C & 1.1167220000 & 1.3391490000 & 0.0651300000 \\
\hline $\mathrm{C}$ & 1.1853550000 & -2.4655990000 & -0.1991280000 \\
\hline $\mathrm{C}$ & 1.3883700000 & -3.3877640000 & 0.8379800000 \\
\hline $\mathrm{H}$ & 1.5722130000 & 90000 & 1.9659810000 \\
\hline & 990000 & -1.06862 & -0 . \\
\hline & 1.9219990 & -3.070472 & 00 \\
\hline & 2.4 & 3.885825 & 1.363 \\
\hline & 2.4581080000 & 5.8274120000 & 0000 \\
\hline C & 2.4859000000 & 1.6357960000 & 0.22 \\
\hline $\mathrm{C}$ & 2.9120840000 & 5.1740230000 & 1.5465070000 \\
\hline $\mathrm{C}$ & 2.9836750000 & 3.0224960000 & 0.4143930000 \\
\hline c & 3.0406950000 & -0.7535170000 & 0.0917750000 \\
\hline $\mathrm{C}$ & 3.4150570000 & 0.5873390000 & 0.2410640000 \\
\hline $\mathrm{H}$ & 3.4373850000 & -2.7890340000 & -1.6933940000 \\
\hline $\mathrm{C}$ & 3.9885910000 & 5.6282040000 & 0.780 \\
\hline $\mathrm{C}$ & 4.0712970000 & 3.4870090000 & -0.3428490000 \\
\hline $\mathrm{C}$ & 4.1010940000 & -1.79242 & 0000 \\
\hline C & 4. & -2 & 00 \\
\hline $\mathrm{H}$ & 4.37 & 00 & 00 \\
\hline $\mathrm{H}$ & 4.4719270000 & 00000 & 520000 \\
\hline $\mathrm{H}$ & 4.5163570000 & 2.8296870000 & -1.0925250000 \\
\hline $\mathrm{C}$ & 4.5670280000 & 4.7791620000 & -0.1651300000 \\
\hline $\mathrm{H}$ & 5.0184170000 & -1.0354180000 & 1.9052880000 \\
\hline $\mathrm{C}$ & 5.0760600000 & -1.7870470000 & 1.1153770000 \\
\hline $\mathrm{C}$ & 5.2021650000 & -3.7236280000 & -0.8821400000 \\
\hline $\mathrm{H}$ & 5.2503880000 & -4.4752330000 & -1.6721550000 \\
\hline $\mathrm{H}$ & 5.4060 & 0000 & -0.7717640000 \\
\hline $\mathrm{C}$ & 6.0945740000 & -2.7404430000 & 1.1330470000 \\
\hline $\mathrm{C}$ & 6.1610460000 & -3.7134220000 & 0.1340470000 \\
\hline $\mathrm{H}$ & 6.8363280000 & -2.7253400000 & 1.9337390000 \\
\hline $\mathrm{H}$ & 6.9567180000 & -4.4602280000 & 0.1462310000 \\
\hline
\end{tabular}

\section{2@SiO 2 -SP}

$\begin{array}{lrrr}\mathrm{H} & -8.3802680000 & -2.4181260000 & -0.0690300000 \\ \mathrm{H} & -7.7507340000 & -0.8147530000 & 1.7340420000 \\ \mathrm{C} & -7.4002520000 & -1.9381840000 & -0.0786480000\end{array}$ 


\begin{tabular}{|c|c|c|c|}
\hline & 450000 & 6980000 & \\
\hline & -6.7583830000 & 0000 & מחمחל \\
\hline & -6.4894210000 & 60000 & 000 \\
\hline & 0000 & 0000 & חת \\
\hline & -5.5 & & 000 \\
\hline & 000 & 00 & 000 \\
\hline & -4.8 & 0 & 000 \\
\hline & 000 & -1.8 & 000 \\
\hline & -4.46 & 1.89 & 000 \\
\hline & 9690000 & 0000 & 000 \\
\hline & -3.9575050000 & 0000 & 000 \\
\hline & -3.54 & -0.0 & 000 \\
\hline & -3.5 & 0 & 00 \\
\hline & -3.45 & 0 & \\
\hline & -3.3 & 0 & \\
\hline & -3.3 & & \\
\hline & -3.2044810000 & -4.9892740000 & 0000 \\
\hline & -2.8 & -2. & 00 \\
\hline & -2.7735290000 & 000 & 000 \\
\hline & -2.7333520000 & 000 & 0000 \\
\hline & -2.6484290000 & 10000 & $=0000$ \\
\hline & -2.4201670000 & 380000 & 000 \\
\hline & -2.337 & 0 & 0 \\
\hline & -2.3337350000 & 240000 & 0000 \\
\hline & 000 & -2.2 & 000 \\
\hline & -2.1 & -5.0 & 00 \\
\hline & -2.1 & -6.0 & 00 \\
\hline & -1.7 & 0 & \\
\hline & 0 & & \\
\hline & & & 00 \\
\hline & 530000 & & 000 \\
\hline & 40000 & & 0000 \\
\hline & 000 & 0000 & 0000 \\
\hline & 000 & 000 & 0000 \\
\hline & 70000 & 000 & 000 \\
\hline & 0000 & 000 & 000 \\
\hline & -0.9 & 0 & 00 \\
\hline & -0.5739350000 & -2.0765100000 & 1.8569960000 \\
\hline & 000 & 0 & 000 \\
\hline & 000 & 0 & 000 \\
\hline & 000 & 0 & 00 \\
\hline & 80000 & 5960000 & 40000 \\
\hline & 000 & -1.48 & \\
\hline & 0.3985750000 & 3.040255 & -1.35 \\
\hline & 1.0049400000 & 1.39 & \\
\hline & 1.1733780000 & 2.0466090000 & 0.697774000 \\
\hline
\end{tabular}

\begin{tabular}{lccc}
$\mathrm{H}$ & 1.3708380000 & -1.7094210000 & 4.0000430000 \\
$\mathrm{H}$ & 1.3889030000 & -3.1413150000 & 2.9414480000 \\
$\mathrm{C}$ & 1.4120800000 & -2.0417940000 & 2.9476110000 \\
$\mathrm{Mo}$ & 1.5572770000 & -1.4792490000 & 0.2134570000 \\
$\mathrm{O}$ & 1.5828400000 & -3.1407350000 & -0.1200280000 \\
$\mathrm{C}$ & 1.5840710000 & 3.7685910000 & -1.4509120000 \\
$\mathrm{H}$ & 1.7391060000 & 4.4427960000 & -2.2952590000 \\
$\mathrm{C}$ & 2.3606130000 & 2.7735360000 & 0.6075900000 \\
$\mathrm{C}$ & 2.5667320000 & 3.6402390000 & -0.4662920000 \\
$\mathrm{C}$ & 2.5888020000 & -1.5271670000 & 2.1358790000 \\
$\mathrm{H}$ & 2.8391850000 & -0.4792740000 & 2.3597690000 \\
$\mathrm{O}$ & 3.1033100000 & -0.5338620000 & -0.4040590000 \\
$\mathrm{H}$ & 3.1328520000 & 2.6430860000 & 1.3655130000 \\
$\mathrm{H}$ & 3.4932110000 & 4.2127550000 & -0.5371650000 \\
$\mathrm{H}$ & 3.5135010000 & -2.1188270000 & 2.0796580000 \\
$\mathrm{H}$ & 3.5211420000 & 1.1715140000 & -2.4662550000 \\
$\mathrm{H}$ & 4.5919240000 & -3.2750830000 & -0.8370550000 \\
$\mathrm{C}$ & 4.6131500000 & 1.3137360000 & -2.4573770000 \\
$\mathrm{Si}$ & 4.7197970000 & -0.2814020000 & -0.2715750000 \\
$\mathrm{H}$ & 4.8309130000 & 2.3071930000 & -2.0341900000 \\
$\mathrm{O}$ & 4.9624900000 & 0.7526220000 & 0.9836430000 \\
$\mathrm{H}$ & 4.9900140000 & 1.2757800000 & -3.4887810000 \\
$\mathrm{O}$ & 5.2716040000 & 0.2889670000 & -1.7113360000 \\
$\mathrm{O}$ & 5.5597350000 & -1.6503460000 & 0.0606220000 \\
$\mathrm{C}$ & 5.5753560000 & -2.7775970000 & -0.8188090000 \\
$\mathrm{H}$ & 5.8491520000 & -2.4801660000 & -1.8434390000 \\
$\mathrm{H}$ & 6.1785810000 & 1.8743880000 & 2.1976730000 \\
$\mathrm{H}$ & 6.2776680000 & 1.1096160000 & 1.4150630000 \\
$\mathrm{H}$ & 6.3234340000 & -3.4874440000 & -0.4410510000 \\
\hline & 6.8061720000 & 0.2376120000 & 1.8305530000 \\
\hline & $\mathbf{3}$ & \\
$\mathrm{H}$ &
\end{tabular}

\section{3@SiO Si-methylidene}

$\begin{array}{lrrr}\mathrm{H} & -5.9855630000 & 1.6416870000 & 0.5193540000 \\ \mathrm{H} & -5.9042030000 & 2.8384070000 & -0.7971890000 \\ \mathrm{H} & -5.5743820000 & 1.1309850000 & -1.1305520000 \\ \mathrm{H} & -5.4368830000 & -1.7265780000 & 1.9832170000 \\ \mathrm{C} & -5.4359370000 & 1.9325230000 & -0.3864120000 \\ \mathrm{H} & -5.1569730000 & -2.8590370000 & 0.6234730000 \\ \mathrm{H} & -5.0791650000 & -1.0867600000 & 0.3513070000 \\ \mathrm{C} & -4.8578160000 & -1.8961290000 & 1.0652300000 \\ \mathrm{C} & -3.9747080000 & 2.1482590000 & -0.0965130000 \\ \mathrm{H} & -3.9681220000 & 0.9689500000 & 1.7075980000 \\ \mathrm{H} & -3.6358720000 & 3.3872870000 & -1.8295090000 \\ \mathrm{O} & -3.4730740000 & -1.8978540000 & 1.4178080000 \\ \mathrm{C} & -3.3670780000 & 1.5662690000 & 1.0178950000 \\ \mathrm{C} & -3.1796790000 & 2.9167780000 & -0.9536530000\end{array}$




\begin{tabular}{|c|c|c|c|}
\hline & 00 & 00 & \\
\hline & 0 & 00 & -1.00 \\
\hline & -2.2823330000 & -2.2990780000 & 0.3712040000 \\
\hline & -2.1793020000 & 0.7063890000 & 3.1738260000 \\
\hline & -2.1534690000 & -3.6264750000 & -2.3437960000 \\
\hline C & -2.0182940000 & -4.2596410000 & -1.4529230000 \\
\hline C & -1.9995040000 & 1.7097320000 & 1.2768220000 \\
\hline & -1.9768230000 & -1.1403890000 & -0.7618860000 \\
\hline & -1.8130180000 & 3.1040880000 & -0.7237220000 \\
\hline & -1.5595670000 & 4.1246470000 & -2.6065220000 \\
\hline C & -1.3953010000 & 1.0460790000 & 2.4851360000 \\
\hline $\mathrm{C}$ & -1.2134720000 & 2.48 & 0.3949120000 \\
\hline $\mathrm{H}$ & -1.1278010000 & -3.0141910000 & 3.1563 \\
\hline $\mathrm{H}$ & -1.0943370000 & 1.3214710000 & -2.6489330000 \\
\hline $\mathrm{C}$ & -1.0060120000 & 3.9522740000 & -1.6735090000 \\
\hline $\mathrm{H}$ & -0.9391840000 & -4.3502250000 & -1.2479410000 \\
\hline $\mathrm{O}$ & -0.8537180000 & -2.3820910000 & 1.1862220000 \\
\hline $\mathrm{H}$ & -0.8035850000 & 0.1639980000 & 2.1948730000 \\
\hline $\mathrm{H}$ & -0.8005660000 & -4.3234010000 & 1.9749540000 \\
\hline $\mathrm{H}$ & -0.77 & 000 & 0000 \\
\hline $\mathrm{H}$ & -0.71 & 000 & 0000 \\
\hline $\mathrm{C}$ & -0.5604070000 & -3.28 & 00 \\
\hline $\mathrm{C}$ & 400000 & 0.506 & 0000 \\
\hline M & 60000 & 50000 & 000000 \\
\hline $\mathrm{H}$ & -0.0425810000 & 3.4824710000 & -1.9185920000 \\
\hline $\mathrm{H}$ & 0.0935940000 & 4.7229360000 & 1.2111010000 \\
\hline $\mathrm{H}$ & 0.1349960000 & 0.4381830000 & -3.7384190000 \\
\hline $\mathrm{C}$ & 0.2433480000 & 2.6558130000 & 0.6427600000 \\
\hline $\mathrm{H}$ & 0.5143010000 & -3.2107660000 & 2.4659290000 \\
\hline O & 0.6788970000 & 0.3491960000 & 0.1255840000 \\
\hline $\mathrm{C}$ & 0.7758330000 & 3.8855760000 & 1.0517060000 \\
\hline $\mathrm{O}$ & 0.8290460000 & -1.8606890000 & -1.8308010000 \\
\hline $\mathrm{C}$ & 1.1359600000 & 1.5805440000 & 0.4612400000 \\
\hline $\mathrm{H}$ & 1. & -0 & 00 \\
\hline $\mathrm{C}$ & 2.1448370000 & 0000 & 0000 \\
\hline $\mathrm{C}$ & 2.5225 & 0000 & 0000 \\
\hline $\mathrm{H}$ & 2.5372940000 & 5.0103820000 & 1.5901670000 \\
\hline $\mathrm{H}$ & 2.7525980000 & 1.4353510000 & -2.1945890000 \\
\hline $\mathrm{C}$ & 2.8619660000 & -0.2907430000 & 2.7543050000 \\
\hline $\mathrm{H}$ & 3.0077700000 & 0.6985360000 & 3.2123230000 \\
\hline $\mathrm{C}$ & 3.0112450000 & 2.9689270000 & 1.065 \\
\hline $\mathrm{H}$ & 3.2010340000 & -1.0560 & 530000 \\
\hline $\mathrm{C}$ & 3.416 & 0.5532 & 0.4326660000 \\
\hline $\mathrm{C}$ & 3.5899800000 & -0.4127920000 & 1.4422370000 \\
\hline $\mathrm{C}$ & 3.8202190000 & 1.3895450000 & -1.9243400000 \\
\hline & 4.0524780000 & 0.3978660000 & -0.8147590000 \\
\hline
\end{tabular}

$\begin{array}{lrrr}\mathrm{H} & 4.0851740000 & 3.0852310000 & 1.2249960000 \\ \mathrm{H} & 4.1128750000 & 2.4088100000 & -1.6315040000 \\ \mathrm{H} & 4.3869360000 & 1.1116410000 & -2.8225860000 \\ \mathrm{C} & 4.4268210000 & -1.5064680000 & 1.1965850000 \\ \mathrm{H} & 4.5678640000 & -2.2510450000 & 1.9855110000 \\ \mathrm{C} & 4.8780800000 & -0.7119330000 & -1.0210280000 \\ \mathrm{C} & 5.0798330000 & -1.6756330000 & -0.0281250000 \\ \mathrm{H} & 5.3685580000 & -3.6757240000 & -0.7898640000 \\ \mathrm{H} & 5.3701350000 & -0.8305420000 & -1.9905790000 \\ \mathrm{C} & 5.9434920000 & -2.8815920000 & -0.2865270000 \\ \mathrm{H} & 6.3358650000 & -3.3025430000 & 0.6495440000 \\ \mathrm{H} & 6.7947490000 & -2.6347890000 & -0.9367270000\end{array}$

\section{3@SiO $\mathbf{S B P}_{2}$-TBP}

\begin{tabular}{llll}
$\mathrm{H}$ & -6.9353120000 & 0.9157440000 & 0.2847890000 \\
$\mathrm{H}$ & -6.2273230000 & 1.7334330000 & 1.7010220000 \\
$\mathrm{C}$ & -6.0147500000 & 1.3580910000 & 0.6905760000 \\
$\mathrm{H}$ & -5.7685000000 & 2.2307080000 & 0.0630510000 \\
$\mathrm{H}$ & -5.5519320000 & -0.6775570000 & -1.0657750000 \\
$\mathrm{C}$ & -4.8831500000 & 0.3657370000 & 0.6986530000 \\
$\mathrm{C}$ & -4.7806900000 & -0.6161410000 & -0.2924300000 \\
$\mathrm{H}$ & -4.4400920000 & -2.3623840000 & -2.1778780000 \\
$\mathrm{H}$ & -3.9549340000 & 1.1653440000 & 2.4720110000 \\
$\mathrm{C}$ & -3.8875970000 & 0.4167770000 & 1.6775860000 \\
$\mathrm{H}$ & -3.7213000000 & -3.5613650000 & -1.0784070000 \\
$\mathrm{C}$ & -3.7155190000 & -1.5213650000 & -0.3341360000 \\
$\mathrm{C}$ & -3.6365220000 & -2.5294380000 & -1.4486300000 \\
$\mathrm{H}$ & -3.2840740000 & 1.3509030000 & -1.8138820000 \\
$\mathrm{C}$ & -2.8049490000 & -0.4702550000 & 1.6803550000 \\
$\mathrm{H}$ & -2.7891590000 & -4.1059130000 & 1.1796970000 \\
$\mathrm{C}$ & -2.7117130000 & -1.4450240000 & 0.6597540000 \\
$\mathrm{H}$ & -2.6720350000 & -2.4539940000 & -1.9722270000 \\
$\mathrm{H}$ & -2.6444960000 & 2.4519700000 & -0.4996120000 \\
$\mathrm{C}$ & -2.4105300000 & 1.8043840000 & -1.3461720000 \\
$\mathrm{H}$ & -2.1426900000 & 0.2135920000 & 3.6132200000 \\
$\mathrm{H}$ & -1.8987510000 & 2.4274810000 & -3.3635970000 \\
$\mathrm{C}$ & -1.7793660000 & -3.7672110000 & 0.9395280000 \\
$\mathrm{C}$ & -1.7670080000 & -0.3731550000 & 2.7648630000 \\
$\mathrm{C}$ & -1.5778250000 & -2.4115100000 & 0.6502910000 \\
$\mathrm{H}$ & -1.4684540000 & -1.3672200000 & 3.1272180000 \\
$\mathrm{C}$ & -1.4107960000 & 2.4882750000 & -2.3865330000 \\
$\mathrm{H}$ & -1.2679550000 & 3.5157300000 & -2.0398210000 \\
$\mathrm{H}$ & -1.1690810000 & -0.4932690000 & -2.3369120000 \\
$\mathrm{H}$ & -0.8976660000 & -5.7238770000 & 1.1721150000 \\
\hline & -0.7582330000 & 0.1206890000 & 2.3884790000 \\
\hline
\end{tabular}




\begin{tabular}{|c|c|c|c|}
\hline & -0.2644040000 & -1.9817140000 & 0.36 \\
\hline 0 & -0.0100010000 & 2.0005770000 & 0.1380030000 \\
\hline & 0.0075290000 & -0.6756420000 & 0.1375820000 \\
\hline C & 0.0445890000 & 1.8738860000 & -2.5632770000 \\
\hline & 0.1968720000 & 1.4172350000 & -3.5417730000 \\
\hline & 0.5420490000 & 4.5680250000 & 0.5638700000 \\
\hline & 0.5729630000 & -4.2285770000 & 0.6518590000 \\
\hline & 0.6889620000 & 6.6006390000 & 0.7956460000 \\
\hline & 0.8210800000 & -2.8838280000 & 0.3613530000 \\
\hline & 0.8337040000 & 2.5625960000 & -2.25 \\
\hline $\mathrm{H}$ & 0.8423330000 & -2.0077450000 & -2.4453700000 \\
\hline S & 1.1396840000 & 3.0430990000 & 0.6327290000 \\
\hline C & 1.3080070000 & 5.7072740000 & 0.9563020000 \\
\hline $\mathrm{H}$ & 1.3832210000 & 1.2902690000 & 3.6543630000 \\
\hline $\mathrm{H}$ & 1.4074530000 & -3.6717280000 & -2.3131870000 \\
\hline $\mathrm{H}$ & 1.4109990000 & -4.9288060000 & 0.6510050000 \\
\hline $\mathrm{H}$ & 1.4477490000 & 0.6332070000 & 1.9923220000 \\
\hline $\mathrm{H}$ & 1.583 & 5.65 & 00 \\
\hline $\mathrm{O}$ & 1.5885110 & 2.72477 & 2.18 \\
\hline $\mathrm{H}$ & 1.5918000000 & -1.6901130000 & 2.728827 \\
\hline $\mathrm{C}$ & 1.7497370000 & -2.6299880000 & -2.4041440000 \\
\hline $\mathrm{C}$ & 1.8513840000 & 1.4061190000 & 10000 \\
\hline $\mathrm{C}$ & 2.1933010000 & -2.3770670000 & 0.0778260000 \\
\hline $\mathrm{H}$ & 2.2261780000 & 5.7961300000 & 0.3532290000 \\
\hline H & 2.2794610000 & -2.5226440000 & -3.3599310000 \\
\hline $\mathrm{O}$ & 2.4328300000 & 2.9970970000 & -0.4027400000 \\
\hline $\mathrm{H}$ & 2.4605770000 & -3.2169940000 & 2.8520910000 \\
\hline $\mathrm{C}$ & 2.5751210000 & -2.1581650000 & 2.5732730000 \\
\hline $\mathrm{H}$ & 2.6175970000 & 0.9204020000 & -0.7130720000 \\
\hline $\mathrm{C}$ & 2.6379530000 & -2.2427300000 & -1.2522110000 \\
\hline $\mathrm{H}$ & 2.936890000 & .2482280000 & 2.7691450 \\
\hline $\mathrm{C}$ & 3.03 & -2.0121030000 & 1.14 \\
\hline $\mathrm{C}$ & +60000 & 1.8236450000 & 70000 \\
\hline $\mathrm{H}$ & 3.2925890000 & -1.6998740000 & 3.2668590000 \\
\hline $\mathrm{H}$ & 3.8723110000 & 1.6569610000 & 0.3223540000 \\
\hline $\mathrm{H}$ & 3.8736120000 & 1.9611910000 & -1.4393240000 \\
\hline $\mathrm{C}$ & 3.9241920000 & -1.7425070000 & -1.4886690000 \\
\hline $\mathrm{H}$ & 4.2692840000 & -1.6442890000 & -2.5219760000 \\
\hline $\mathrm{C}$ & 4.3130490000 & -1.5134580000 & 0.8668010000 \\
\hline $\mathrm{C}$ & 4.7758730000 & -1.3670630000 & -0.4451800000 \\
\hline $\mathrm{H}$ & 4.9640870000 & -1.2310560000 & 1.6994720000 \\
\hline $\mathrm{H}$ & 6.1176970000 & 0.3090330000 & -0.7060790000 \\
\hline $\mathrm{C}$ & 6.1397660000 & -0.7930180000 & -0.7242980000 \\
\hline $\mathrm{H}$ & 6.5076390000 & -1.0967720000 & -1.7139240000 \\
\hline $\mathrm{H}$ & 6.8731410000 & -1.1139570000 & 0.0290570000 \\
\hline
\end{tabular}

3@SiO ${ }_{2}$-SP

\begin{tabular}{|c|c|c|c|}
\hline & 00 & 00 & \\
\hline & 0 & 00 & -0.5410820000 \\
\hline & 5.0152170000 & -3.4480480000 & 0000 \\
\hline & -5.8175600000 & -1.5892150000 & -1.5114730000 \\
\hline & -5.5319470000 & 0000 & 1.0955740000 \\
\hline & -5.2477570000 & -1.3053580000 & -0.6221920000 \\
\hline & -5.2277750000 & -2.1650160000 & 0.4799570000 \\
\hline & -5.1394860000 & 1.7008130000 & -1.6921740000 \\
\hline & -5.0280720000 & 0.2436640000 & -2.7030210000 \\
\hline & -4.7201060000 & 2.8034320000 & 10000 \\
\hline$c$ & -4.5683760000 & 0.7748850000 & -1.8592370000 \\
\hline $\mathrm{C}$ & -4.5423450000 & -0.0973460000 & -0.6306560000 \\
\hline c & -4.4716050000 & -1.7869610000 & 1.5941650000 \\
\hline $\mathrm{H}$ & -4.4396630000 & -2.4450610000 & 20000 \\
\hline $\mathrm{C}$ & -3.7855740000 & 0.2619400000 & 0.5034520000 \\
\hline $\mathrm{C}$ & -3.7486720000 & -0.5899140000 & 1.6265370000 \\
\hline $\mathrm{C}$ & -3.6343110000 & 2.7749270000 & 0.5866020000 \\
\hline $\mathrm{H}$ & -3.5551140000 & 1.0856770000 & 570000 \\
\hline $\mathrm{H}$ & -3.4074490000 & 4.9220680000 & 0.6272420000 \\
\hline $\mathrm{H}$ & -3.1 & 0000 & 0000 \\
\hline $\mathrm{H}$ & -3.0 & 000 & 00 \\
\hline $\mathrm{c}$ & 680000 & 0000 & 00 \\
\hline $\mathrm{c}$ & 130000 & 0000 & 00 \\
\hline $\mathrm{C}$ & -2.8994200000 & 3.95 & 0.5 \\
\hline $\mathrm{H}$ & -2.1297770000 & -1.0767360000 & -2.1875370000 \\
\hline $\mathrm{H}$ & -2.0673130000 & -1.9809410000 & -0.6337170000 \\
\hline $\mathrm{H}$ & -1.8594580000 & -0.3454720000 & 2.6383970000 \\
\hline $\mathrm{C}$ & -1.5989660000 & 1.5001250000 & 0.3612110000 \\
\hline $\mathrm{C}$ & -1.5123820000 & 3.9113070000 & 0.4056080000 \\
\hline$C$ & -1.5078690000 & -1.7118260000 & -1.5398540000 \\
\hline $\mathrm{H}$ & -1.2677740000 & -3.8558200000 & -2.1141100000 \\
\hline $\mathrm{O}$ & -0.9810620000 & 0.2899210000 & 0.3223840000 \\
\hline $\mathrm{H}$ & -0.9279700000 & 4.8330370000 & 0.3773460000 \\
\hline $\mathrm{C}$ & -0.8385870000 & 6882450000 & 0.3088870000 \\
\hline $\mathrm{C}$ & -( & 0 & 50000 \\
\hline $\mathrm{H}$ & 350000 & 20000 & 10000 \\
\hline $\mathrm{H}$ & -0.4593920000 & 2.6357830000 & -2.3531150000 \\
\hline $\mathrm{H}$ & -0.0028990000 & 2.7401400000 & 2.8885700000 \\
\hline $\mathrm{H}$ & 0.1163500000 & 4.3024510000 & -2.2444500000 \\
\hline Mo & 0.2481170000 & -0.5887690000 & -0.8824350000 \\
\hline $\mathrm{H}$ & 0.3173710000 & 1.0252270000 & 2.6092360000 \\
\hline $\mathrm{H}$ & 20000 & -3.2237670000 & -0.27 \\
\hline $\mathrm{C}$ & 0.4466460000 & 3.2519820000 & -2.2768220000 \\
\hline $\mathrm{C}$ & 0.4632350000 & -2.7372220000 & -1.2543050000 \\
\hline $\mathrm{C}$ & 0.6442260000 & 2.6347270000 & 0.1797880000 \\
\hline $\mathrm{C}$ & 0.6679440000 & 0.0177710000 & -2.4093010000 \\
\hline
\end{tabular}




$\begin{array}{lrrr}\mathrm{C} & 0.7894330000 & 2.0207480000 & 2.6366020000 \\ \mathrm{H} & 1.0350550000 & 3.1088810000 & -3.1920110000 \\ \mathrm{C} & 1.2607760000 & 2.9016870000 & -1.0597540000 \\ \mathrm{C} & 1.4331220000 & 2.3390560000 & 1.3146150000 \\ \mathrm{H} & 1.4605340000 & -2.9612010000 & -1.6578280000 \\ \mathrm{H} & 1.5317660000 & 2.0218310000 & 3.4456190000 \\ \mathrm{O} & 1.7876690000 & -0.8950950000 & 0.2339890000 \\ \mathrm{C} & 2.6584560000 & 2.8422550000 & -1.1475370000 \\ \mathrm{O} & 2.7877370000 & -3.2360350000 & 0.9998010000 \\ \mathrm{C} & 2.8218590000 & 2.3194820000 & 1.1867900000 \\ \mathrm{H} & 2.8600270000 & -0.1984810000 & 2.8961180000 \\ \mathrm{H} & 3.1322180000 & 3.0224040000 & -2.1164260000 \\ \mathrm{Si} & 3.1752090000 & -1.7501220000 & 0.4131450000 \\ \mathrm{H} & 3.2936250000 & -5.0991530000 & 1.6934190000 \\ \mathrm{H} & 3.4304850000 & 2.0943160000 & 2.0658730000 \\ \mathrm{C} & 3.4561790000 & 2.5563490000 & -0.0381090000\end{array}$

$\begin{array}{lrrr}\mathrm{H} & 3.5937570000 & -0.1216290000 & -1.9683220000 \\ \mathrm{H} & 3.7581700000 & -1.6776590000 & 3.3559030000 \\ \mathrm{C} & 3.7745870000 & -4.2519860000 & 1.1858520000 \\ \mathrm{C} & 3.8244430000 & -0.7236580000 & 2.8071930000 \\ \mathrm{O} & 3.9755230000 & -1.9483000000 & -1.0036750000 \\ \mathrm{H} & 4.1749940000 & -4.5933930000 & 0.2183460000 \\ \mathrm{O} & 4.1783570000 & -0.9379520000 & 1.4415410000 \\ \mathrm{C} & 4.3991190000 & -0.8602920000 & -1.8280310000 \\ \mathrm{H} & 4.6076570000 & -0.1052860000 & 3.2677120000 \\ \mathrm{H} & 4.6124350000 & -3.8941850000 & 1.8076550000 \\ \mathrm{H} & 4.6771220000 & -1.2703900000 & -2.8083660000 \\ \mathrm{C} & 4.9560160000 & 2.4954470000 & -0.1395800000 \\ \mathrm{H} & 5.2745370000 & -0.3570950000 & -1.3889100000 \\ \mathrm{H} & 5.2896080000 & 2.5318760000 & -1.1854060000 \\ \mathrm{H} & 5.3390030000 & 1.5698870000 & 0.3159080000 \\ \mathrm{H} & 5.4245620000 & 3.3391490000 & 0.3913550000\end{array}$




\section{Experimental Procedures and Catalytic Data}

Metathesis of cis-4-nonene:
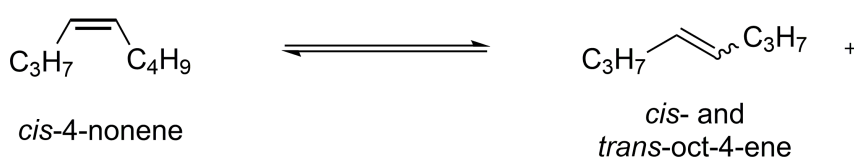

$\mathrm{C}_{4} \mathrm{H}_{9} \curvearrowright \mathrm{N}_{4} \mathrm{C}_{9}$

$\mathrm{C}_{3} \mathrm{H}_{7} \curvearrowright \mathrm{C}_{4} \mathrm{H}_{9}$

trans-oct-4-ene

trans-dec-5-ene

trans-4-nonene

Every dispensing step for experiments with cis-4-nonene was performed manually inside a nitrogen-filled glovebox $\left(<0.1 \mathrm{ppm} \mathrm{H}_{2} \mathrm{O}\right.$ and $\left.\mathrm{O}_{2}\right)$. At $\mathrm{t}=0$, a $0.95 \mathrm{M}$ solution of cis-4-nonene in toluene containing decaline as internal standard $(0.1 \mathrm{M})$ was added to the catalyst placed inside a $10 \mathrm{~mL}$ screw cap vial containing a magnetic stirrer, and the reaction was stirred at $620 \mathrm{rpm}$. The reaction mixtures were agitated for 1 hour and $10 \mu \mathrm{L}$ aliquots were taken after ca. 3, 5, 10, 30 and $60 \mathrm{~min}$ after the addition of cis-4-nonene. The aliquots were dispensed into GC vials containing $500 \mu \mathrm{L}$ of a solution of 4-heptanone in toluene (ca $5.6 \cdot 10^{-4} \mathrm{M}$ ) for quenching. The resulting solution was analyzed by GC/FID (Agilent Technologies 7890 A) equipped with an HP-5 (Agilent Technologies) column. Quantification gave conversion and $(E) /(Z)$ selectivity values for oct-4-ene and dec-5-ene. Equilibrium conversion was reached at ca. 50\%.

Metathesis of 1-nonene:

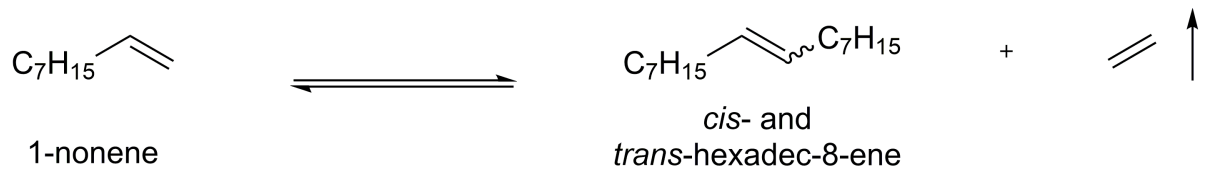

Every agitation and dispensing step for experiments with 1-nonene were performed by the liquid handling robot (Zinsser Analytics) operated inside a nitrogen-filled glovebox $(<0.1 \mathrm{ppm}$ $\mathrm{H}_{2} \mathrm{O}$ and $\mathrm{O}_{2}$ ). At $\mathrm{t}=0$, a $0.95 \mathrm{M}$ solution of 1-nonene in toluene containing decaline as internal standard $(0.1 \mathrm{M})$ was added to the catalyst introduced in a $10 \mathrm{~mL}$ vial and the reaction mixture was agitated at $300 \mathrm{rpm}$. Catalytic tests were conducted open to the glovebox atmosphere. The reaction mixtures were agitated for 8 hours and $30 \mu \mathrm{L}$ aliquots were taken after ca. 3, 10, 30, $60,120,240$ and $480 \mathrm{~min}$ after the addition of 1-nonene. The aliquots were dispensed into GC vials containing $500 \mu \mathrm{L}$ of a solution of 4-heptanone in toluene (ca $5.6 \cdot 10^{-4} \mathrm{M}$ ) for quenching. The resulting solution was analyzed by GC/FID (Agilent Technologies 7890 A) equipped with an HP-5 (Agilent Technologies) column. Quantification gave conversion and $(E) /(Z)$ selectivity values for hexadec-8-ene. Equilibrium conversion is reached at ca. $100 \%$ in our conditions.

$X($ substrate $)=\frac{n(\text { substrate }) \text { initial }-n(\text { substrate })}{n(\text { substrate }) \text { initial }} \quad \times 100 \%$ 


$$
\begin{aligned}
& \mathrm{TOF}_{3 \min }=\frac{X(\text { substrate }) \text { at } 3 \mathrm{~min}}{100 \times 3 \mathrm{~min}} \times \frac{n(\text { substrate })}{n(\text { catalyst })}, \text { in }\left[\mathrm{min}^{-1}\right] \\
& S_{\mathrm{C} 16}(E / Z)=\frac{n(\mathrm{C} 16 E) / n(\mathrm{C} 16)}{(n(\mathrm{C} 16)-(n(\mathrm{C} 16 E)) / n(\mathrm{C} 16))}
\end{aligned}
$$

Table S1. Homometathesis of $c i s-4$-nonene (toluene, $30{ }^{\circ} \mathrm{C}$ ).

\begin{tabular}{cccc}
\hline Catalyst & mol\% & TOF $_{3 \mathrm{~min}}{ }^{a}$ & Time to equilibrium conversion \\
\hline $\mathbf{1}$ & 0.1 & $170(52 \%)$ & $3 \mathrm{~min}$ \\
$\mathbf{1}$ & 0.02 & $870(52 \%)$ & $3 \mathrm{~min}$ \\
$\mathbf{1}$ & 0.014 & $250(11 \%)$ & $11 \%$ after $1 \mathrm{~h}$ \\
$\mathbf{2}$ & 0.1 & $1(<1 \%)$ & $4 \%$ after $1 \mathrm{~h}$ \\
$\mathbf{1} @ \mathrm{SiO}_{2}$ & 0.1 & $170(51 \%)$ & $3 \mathrm{~min}$ \\
$\mathbf{1} @ \mathrm{SiO}_{2}$ & 0.02 & $725(44-46 \%)$ & $5-10 \mathrm{~min}$ \\
$\mathbf{1} @ \mathrm{SiO}_{2}$ & 0.014 & $680(29 \%)$ & $10 \mathrm{~min}$ \\
$\mathbf{1} @ \mathrm{SiO}_{2}$ & 0.005 & $100(1 \%)$ & $41 \% \mathrm{after} 9 \mathrm{~h}$ \\
$\mathbf{2} @ \mathrm{SiO}_{2}$ & 0.1 & $170(52 \%)$ & $3 \mathrm{~min}$ \\
$\mathbf{2} @ \mathrm{SiO}_{2}$ & 0.02 & $350(24 \%)$ & $30 \mathrm{~min}$ \\
\hline
\end{tabular}

${ }^{a}$ TOF at 3 min, given in min $^{-1}$ with the corresponding conversions given in brackets. ${ }^{b}$ Performed in duplicate and averaged.

Table S2. Homometathesis of 1-nonene (toluene, $30^{\circ} \mathrm{C}$ ).

\begin{tabular}{cccc}
\hline Catalyst & mol\% & TOF $_{3 \min }{ }^{a}$ & Time to maximum conversion \\
\hline $\mathbf{1}$ & 0.1 & $210(62 \%)$ & $97 \%$ after $4 \mathrm{~h}$ \\
$\mathbf{1}$ & 0.02 & $940(58 \%)$ & $97 \%$ after $4 \mathrm{~h}$ \\
$\mathbf{1}$ & 0.01 & $1270(38 \%)$ & $50 \%$ after $4 \mathrm{~h}$ \\
$\mathbf{2}$ & 0.1 & $30(9 \%)$ & $62 \%$ after $4 \mathrm{~h}$ \\
$\mathbf{1} @ \mathrm{SiO}_{2}$ & 0.1 & $70(22 \%)$ & $97 \%$ after $4 \mathrm{~h}$ \\
$\mathbf{1} @ \mathrm{SiO}_{2}$ & 0.02 & $170(10 \%)$ & $73 \%$ after $8 \mathrm{~h}$ \\
$\mathbf{2} @ \mathrm{SiO}_{2}$ & 0.1 & $40(12 \%)$ & $96 \%$ after $4 \mathrm{~h}$ \\
$\mathbf{2} @ \mathrm{SiO}_{2}$ & 0.02 & $70(5 \%)$ & $95 \%$ after $8 \mathrm{~h}$ \\
\hline
\end{tabular}


${ }^{a} \mathrm{TOF}$ at $3 \mathrm{~min}$, given in $\mathrm{min}^{-1}$ with the corresponding conversions given in brackets.

Plots of Conversion vs Time and Selectivity vs Time for Cis4-Nonene Experiments
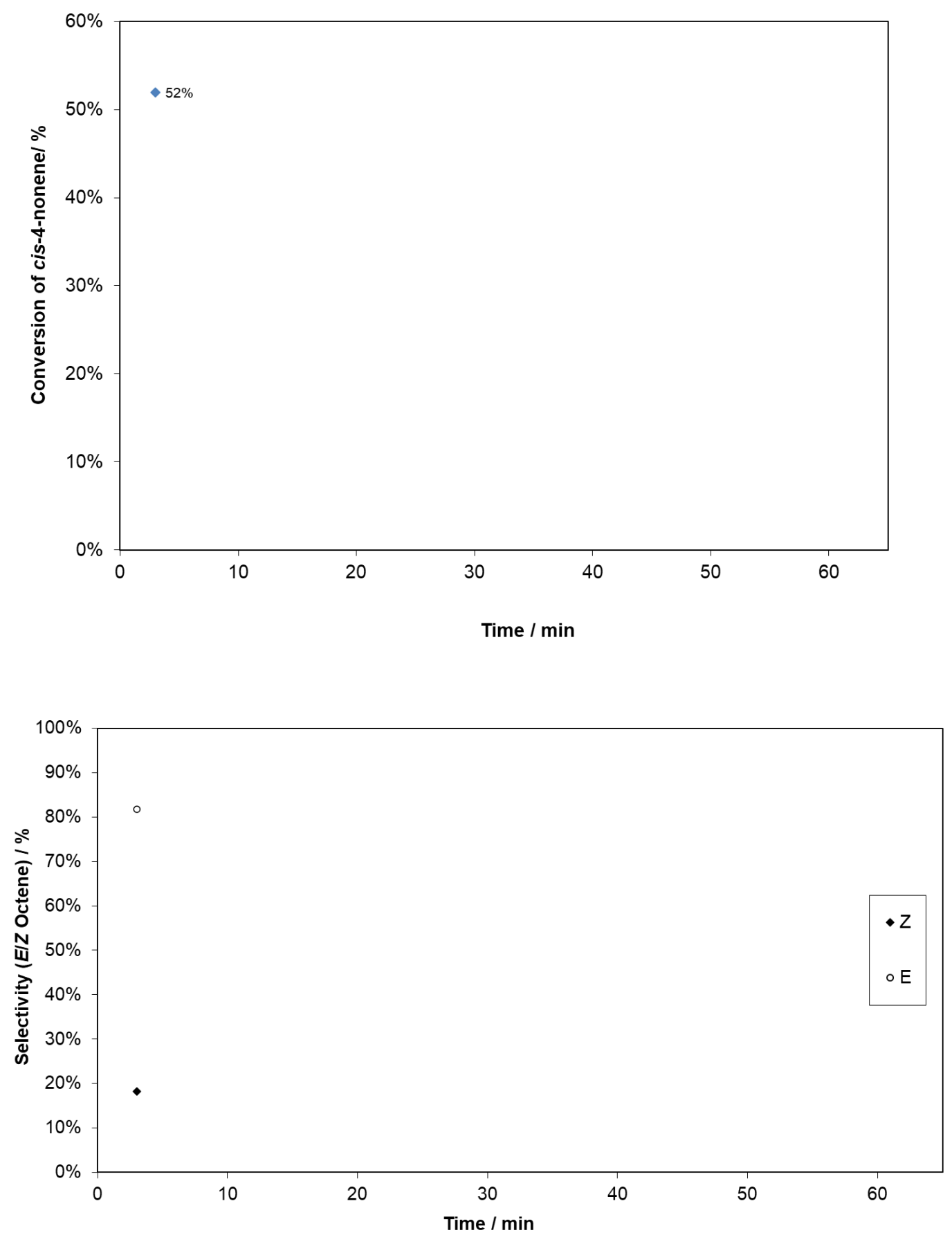


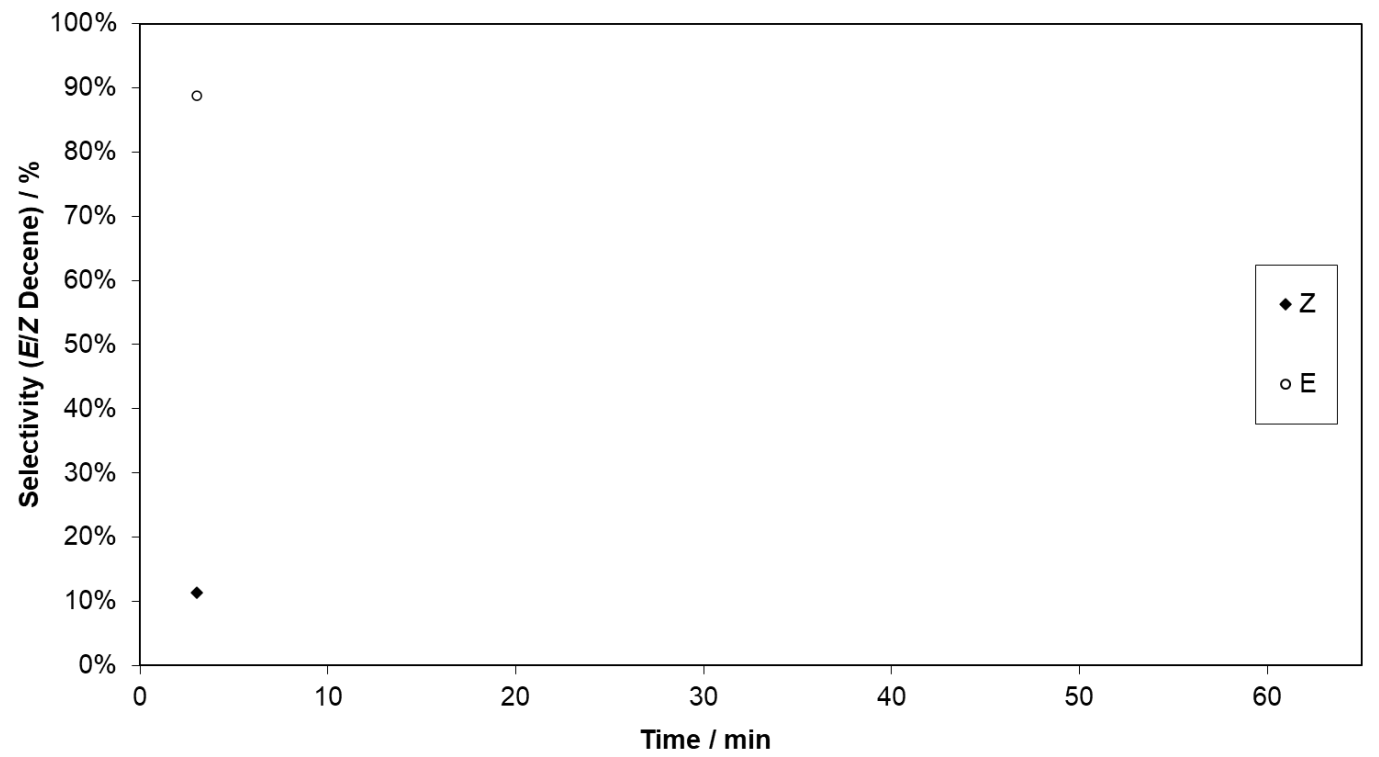

Figure S18. Conversion vs time and selectivity vs time plots for $1(0.1 \mathrm{~mol} \%)$. Due to fast conversion, only one data point could be obtained. 
Supporting Information
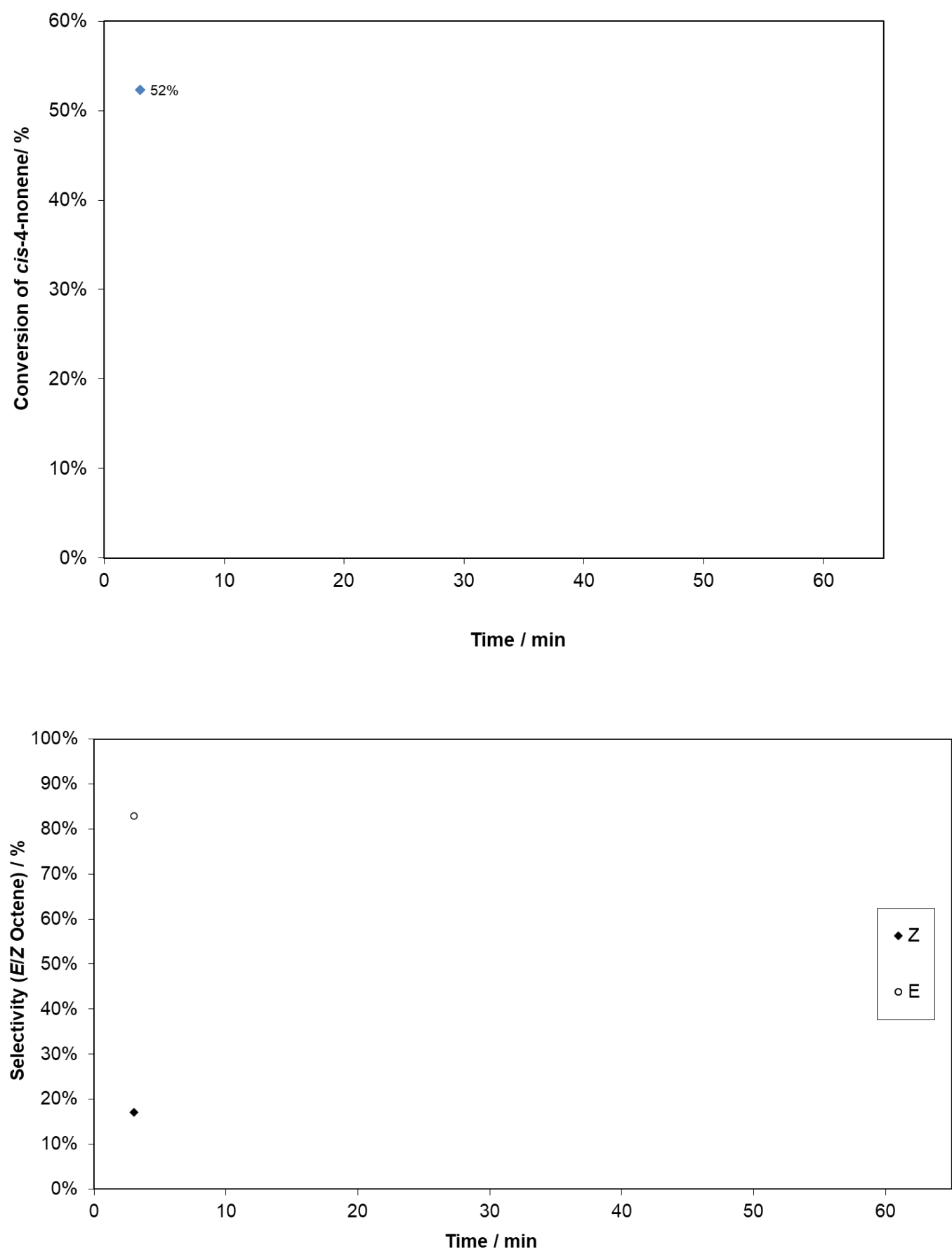


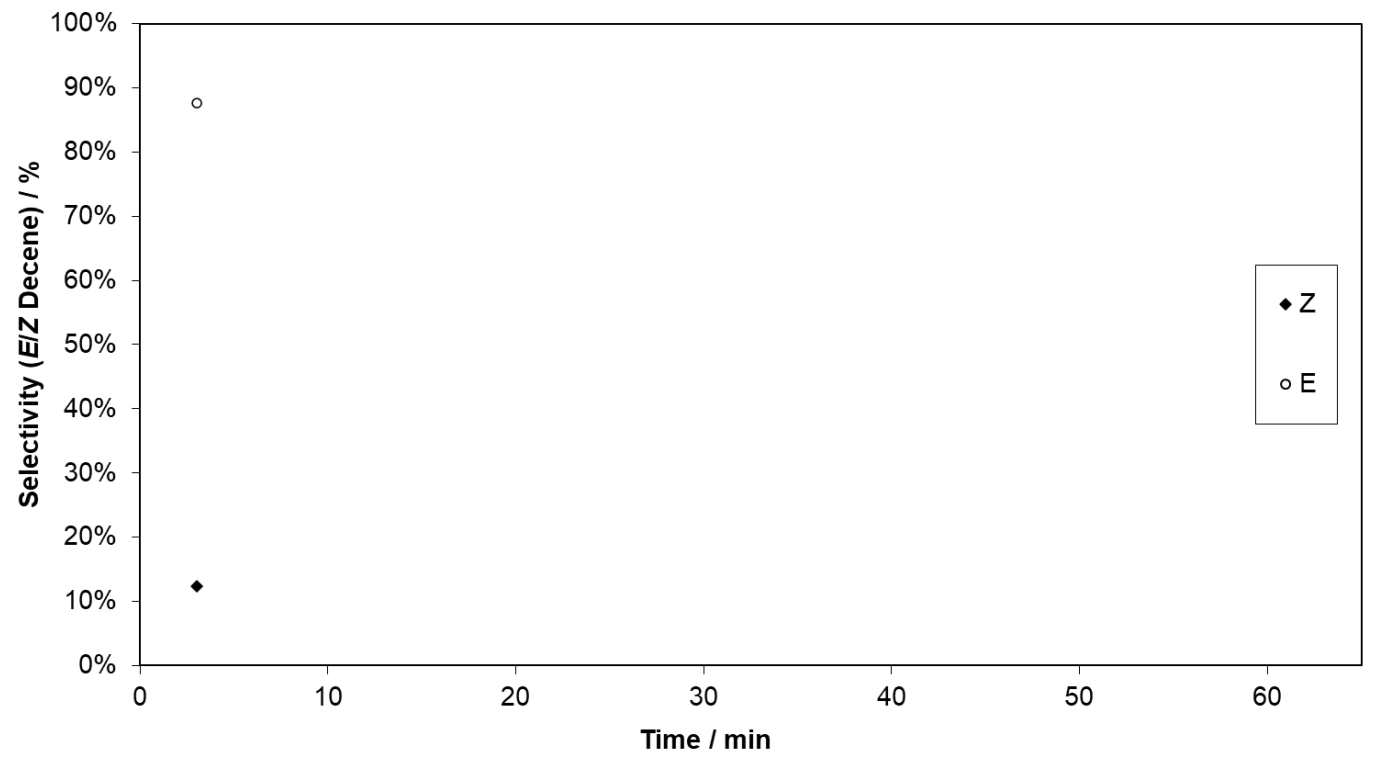

Figure S19. Conversion vs time and selectivity vs time plots for $1(0.02 \mathrm{~mol} \%)$. Due to fast conversion, only one data point could be obtained. 

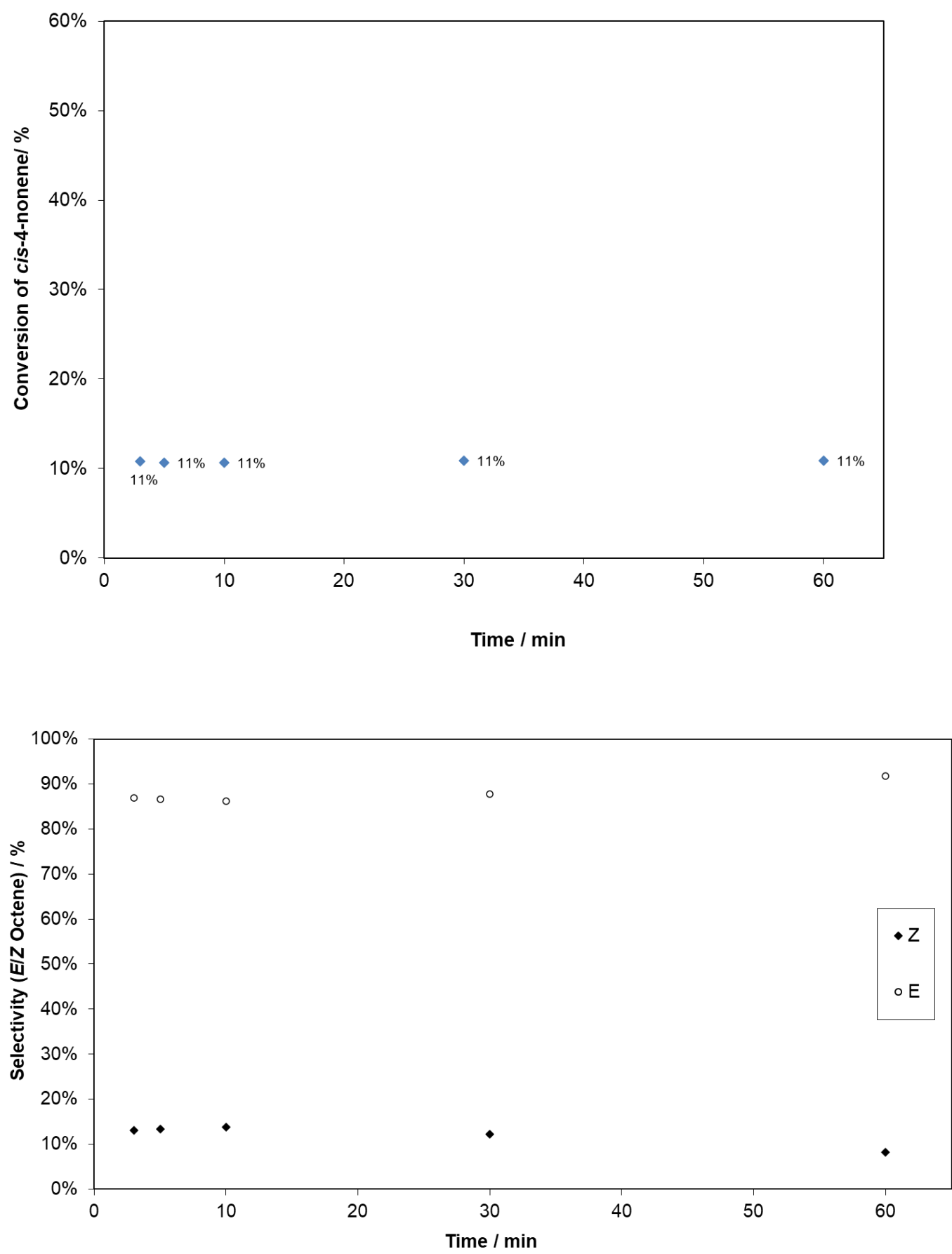


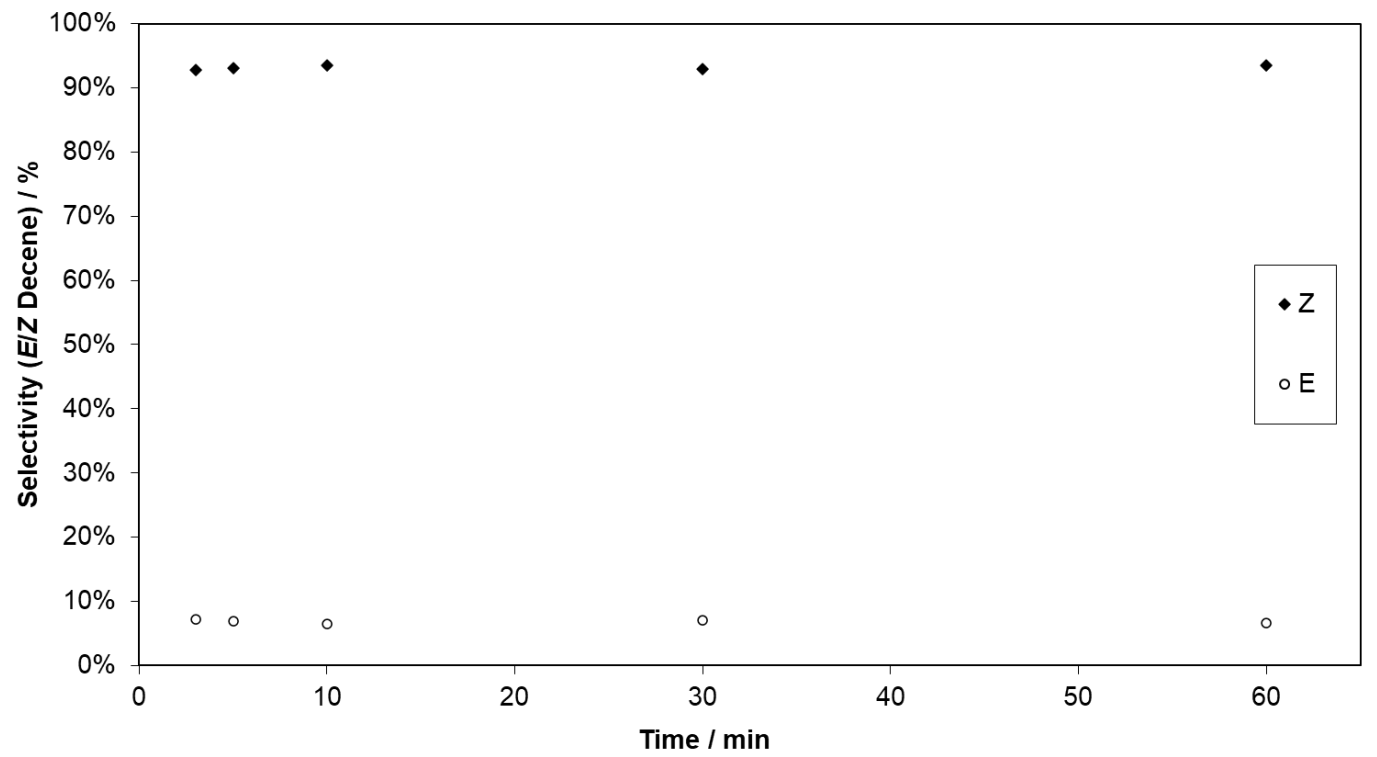

Figure S20. Conversion vs time and selectivity vs time plots for $\mathbf{1}(0.014 \mathrm{~mol} \%)$. 

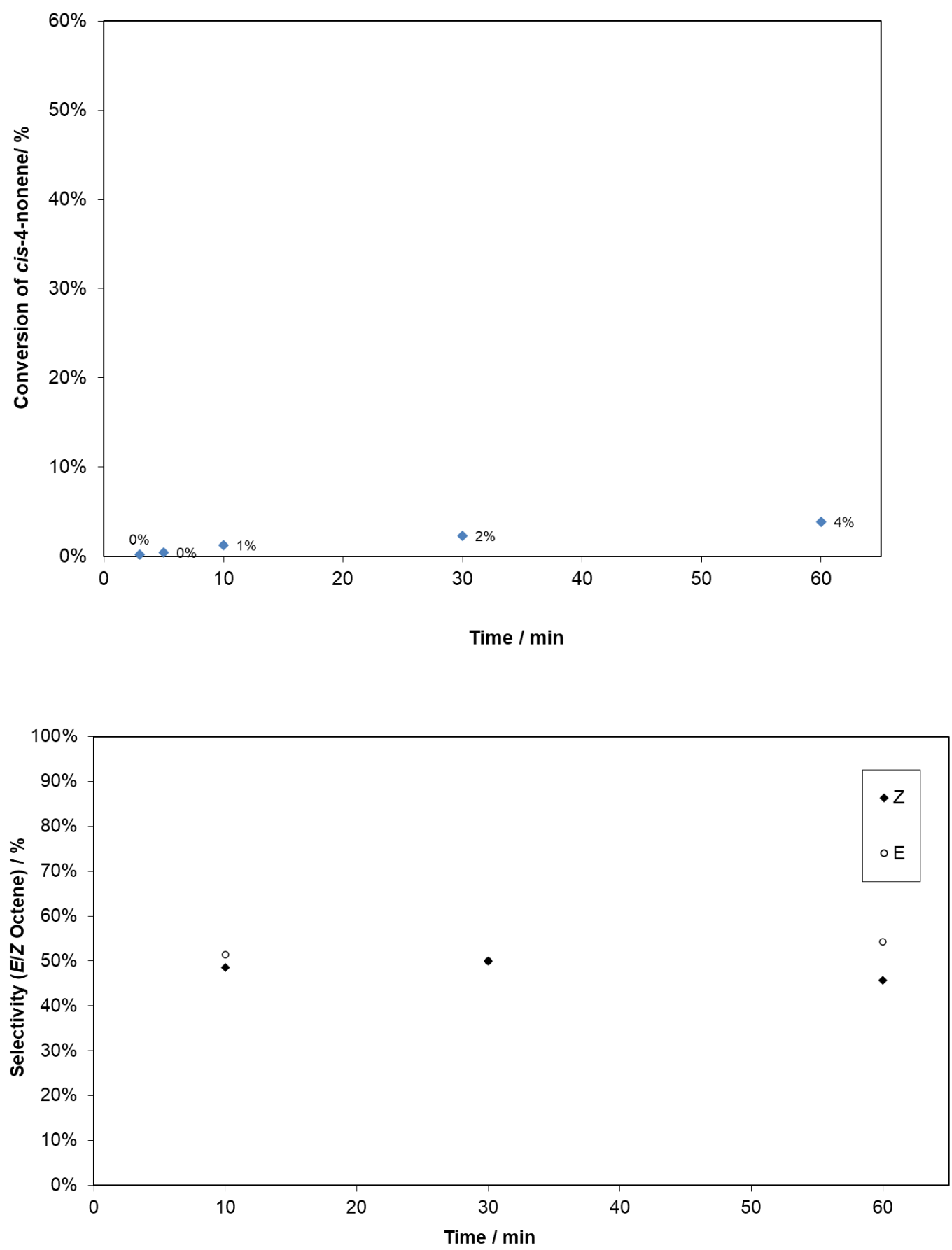


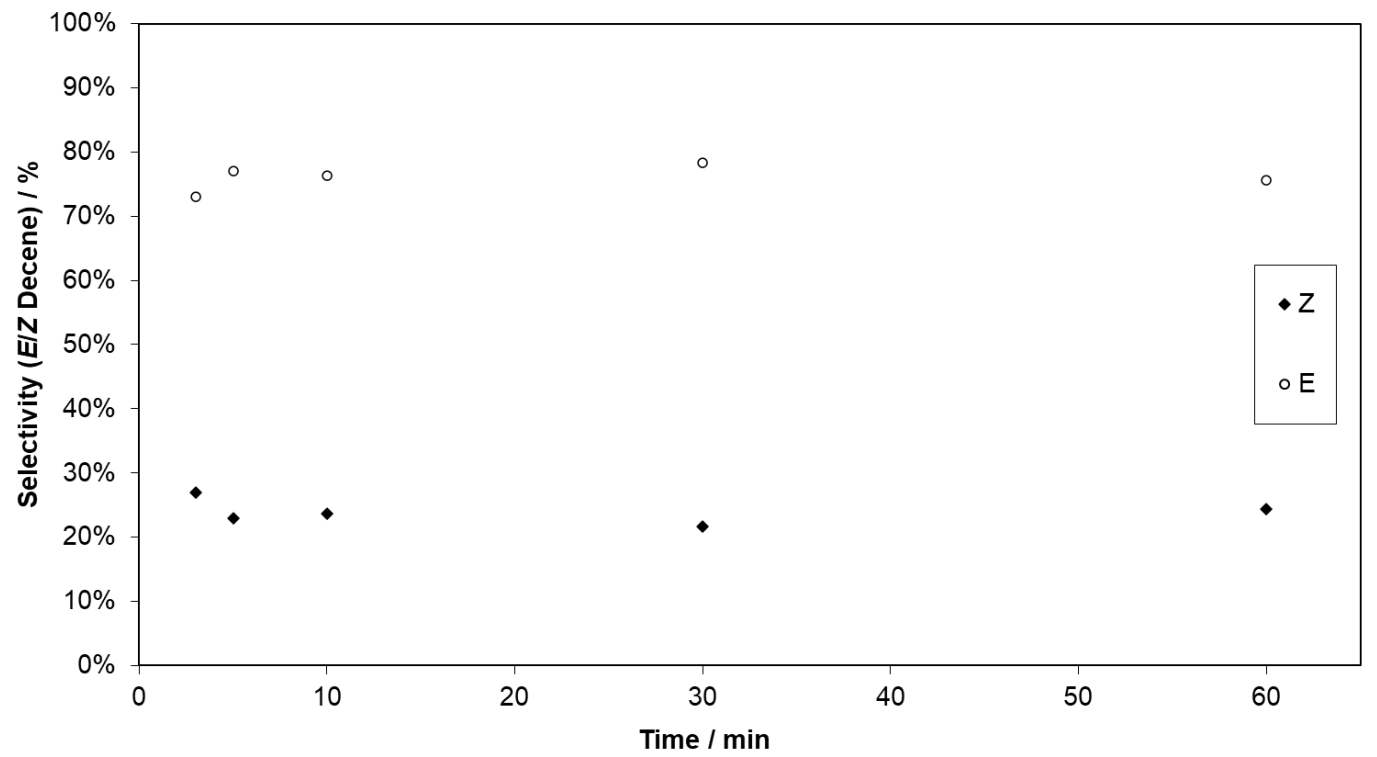

Figure S21. Conversion vs time and selectivity vs time plots for $\mathbf{2}(0.1 \mathrm{~mol} \%)$. 
Supporting Information
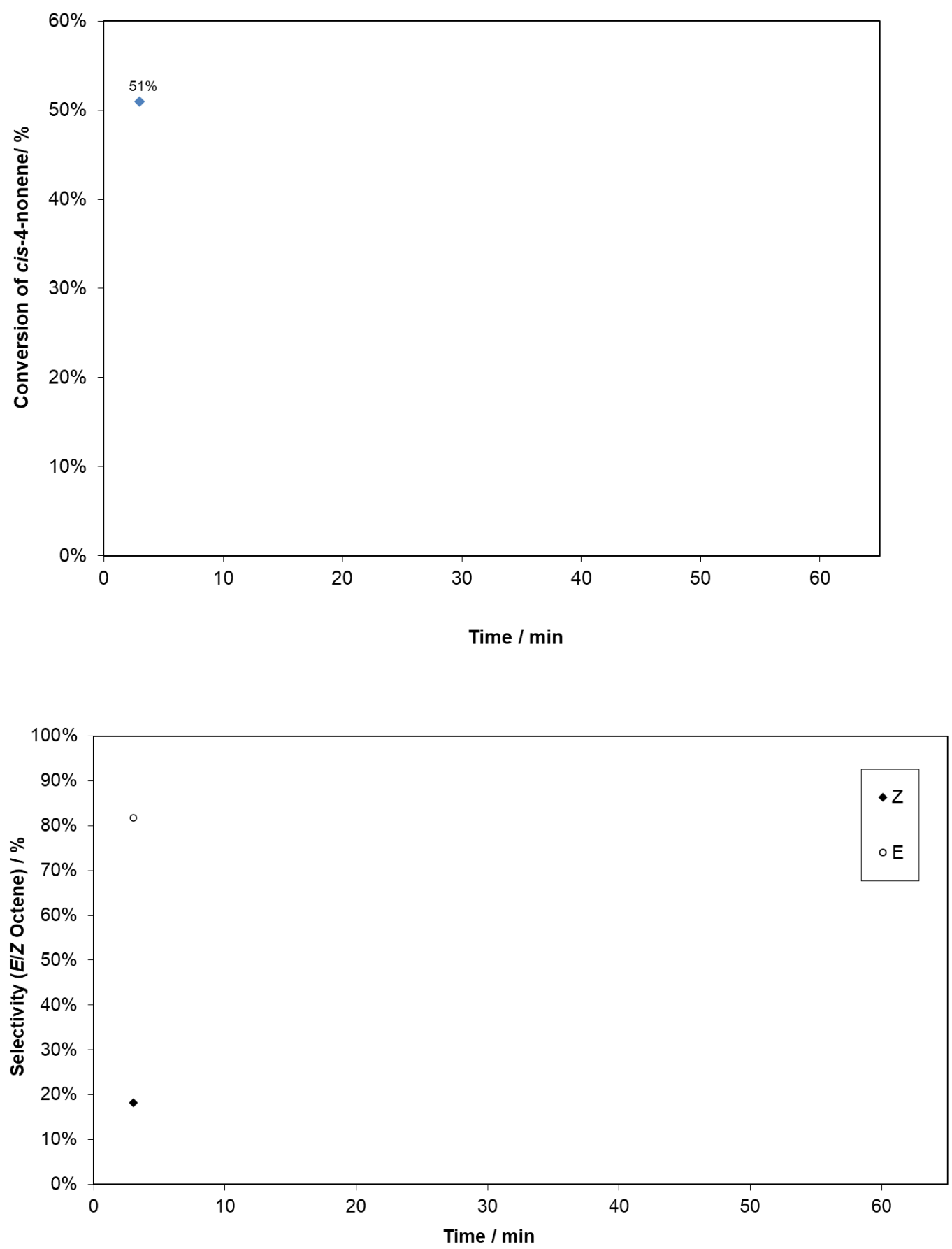


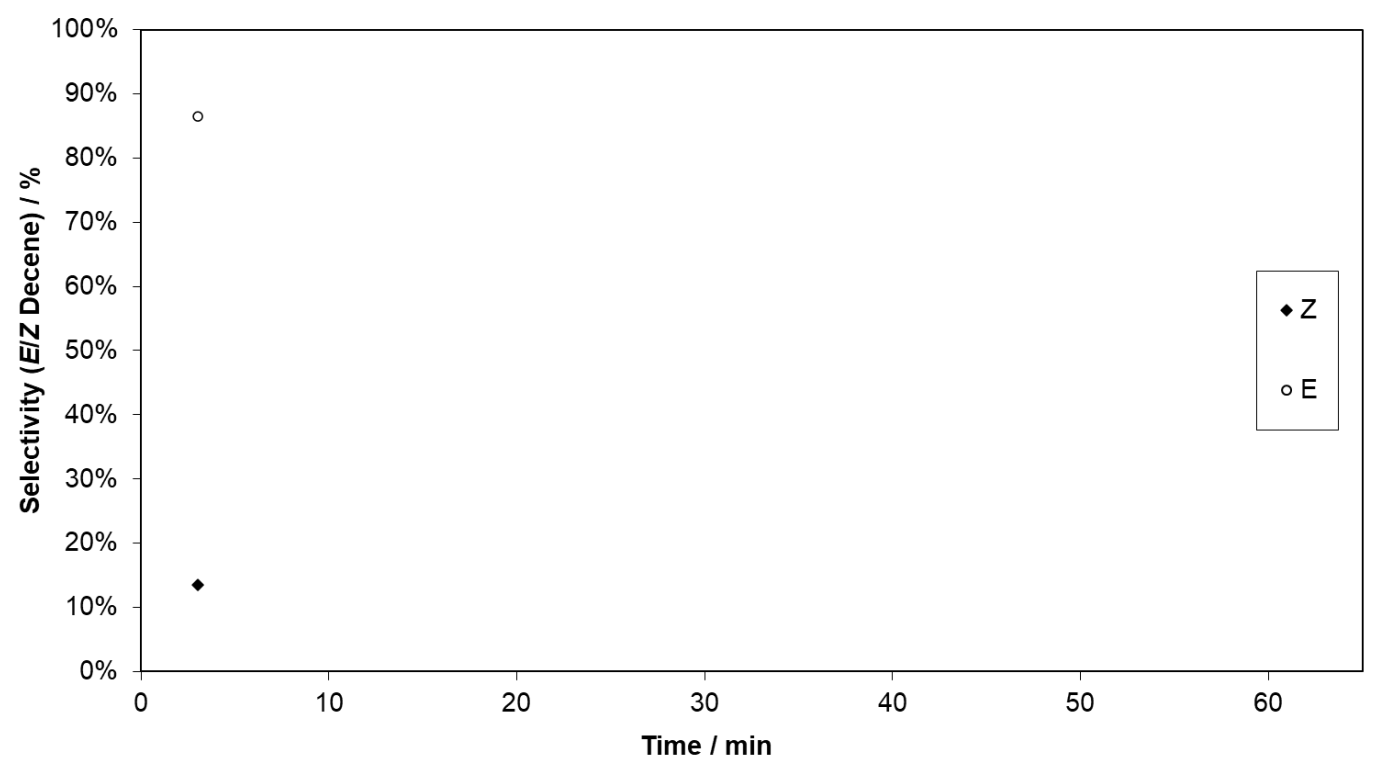

Figure S22. Conversion vs time and selectivity vs time plots for $1 @ \mathrm{SiO}_{2}(0.1 \mathrm{~mol} \%)$. Due to fast conversion, only one data point could be obtained. 
Supporting Information
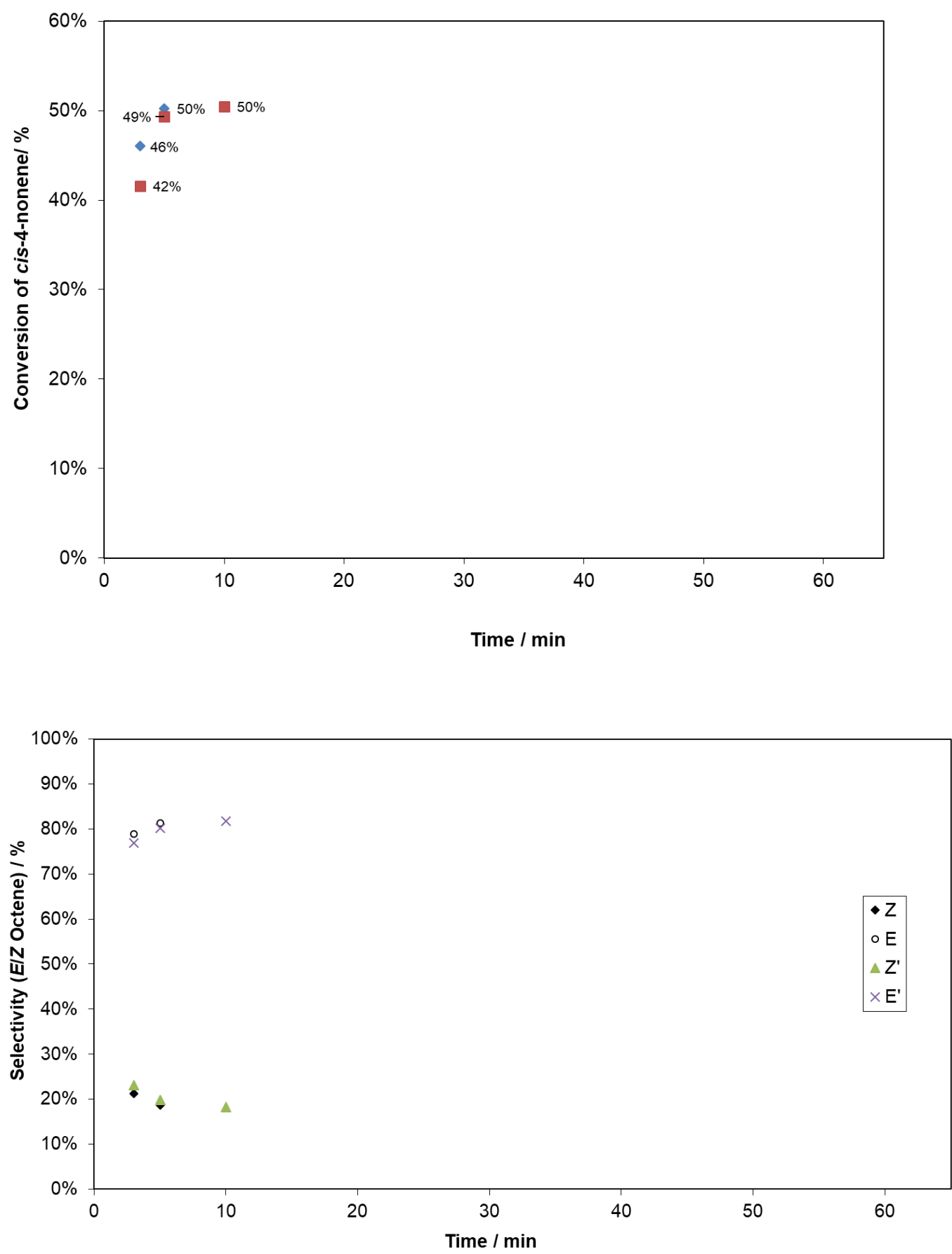


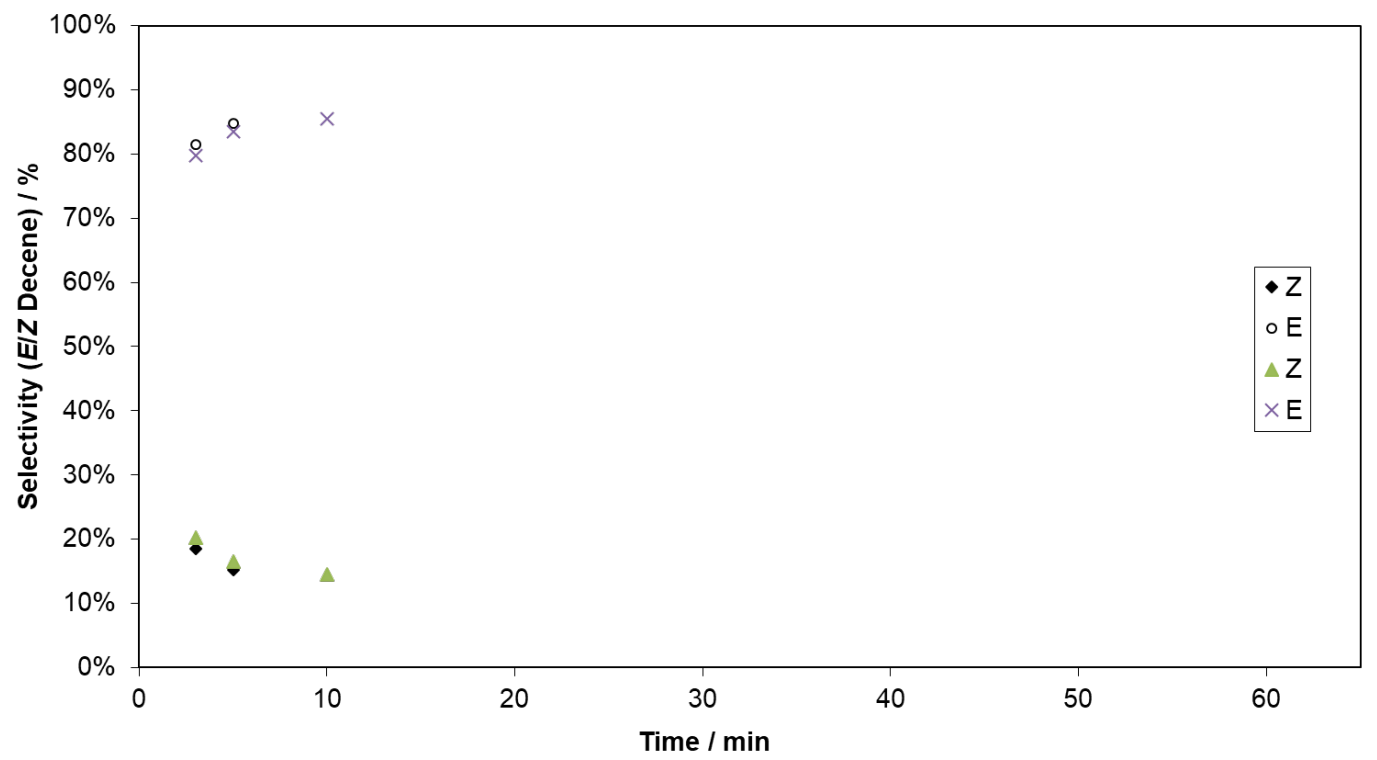

Figure S23. Conversion vs time and selectivity vs time plots for duplicates of $\mathbf{1} @ \mathrm{SiO}_{2}(0.02$ $\mathrm{mol} \%)$. 
Supporting Information
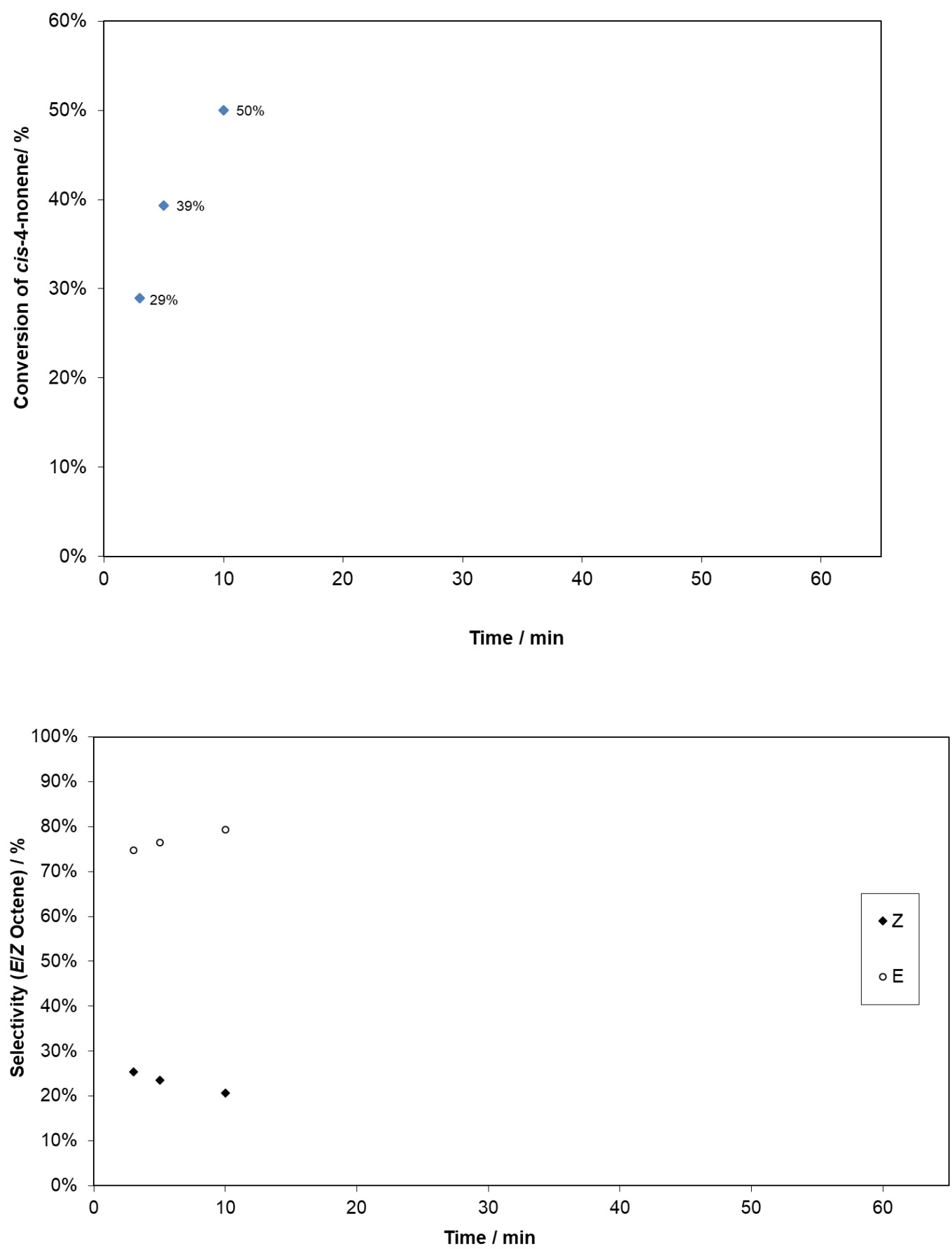


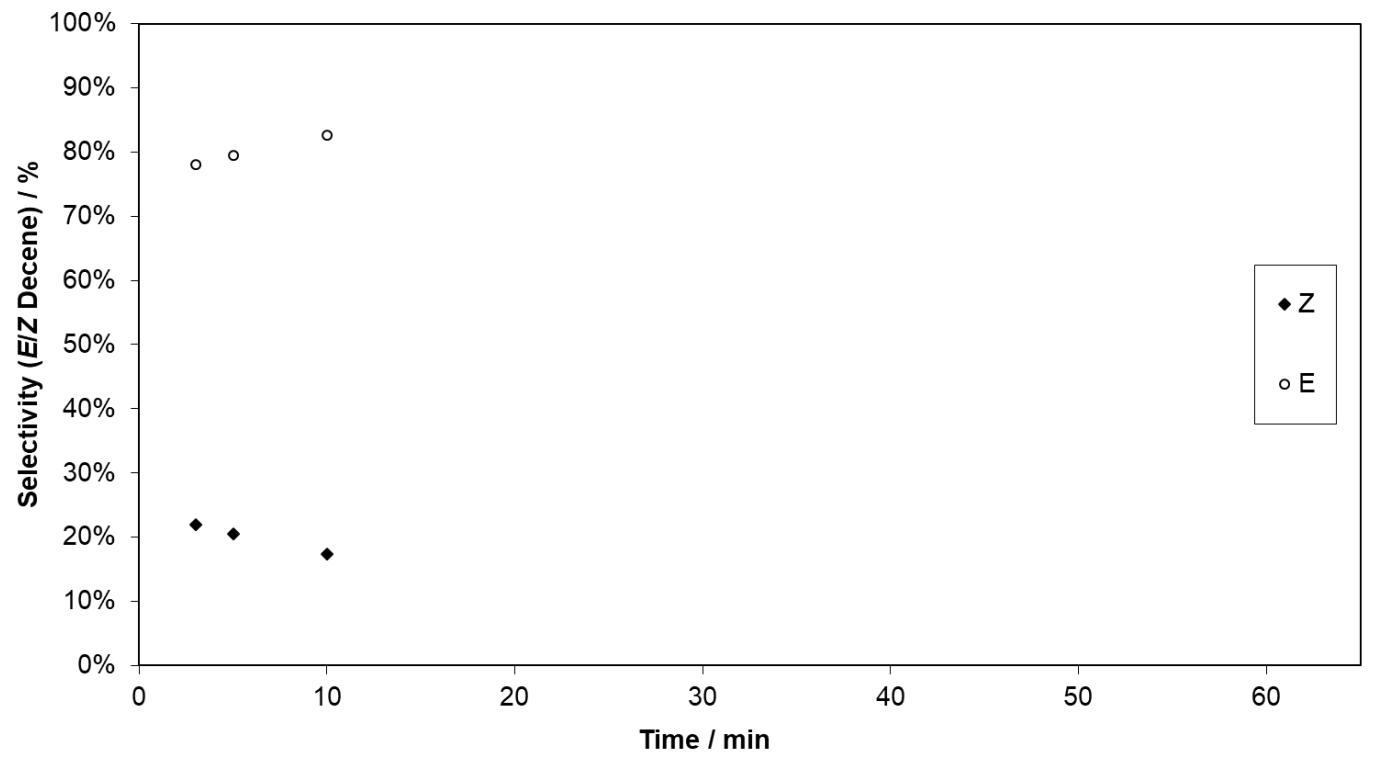

Figure S24. Conversion vs time and selectivity vs time plots for $\mathbf{1} @ \mathrm{SiO}_{2}(0.014 \mathrm{~mol} \%)$. 

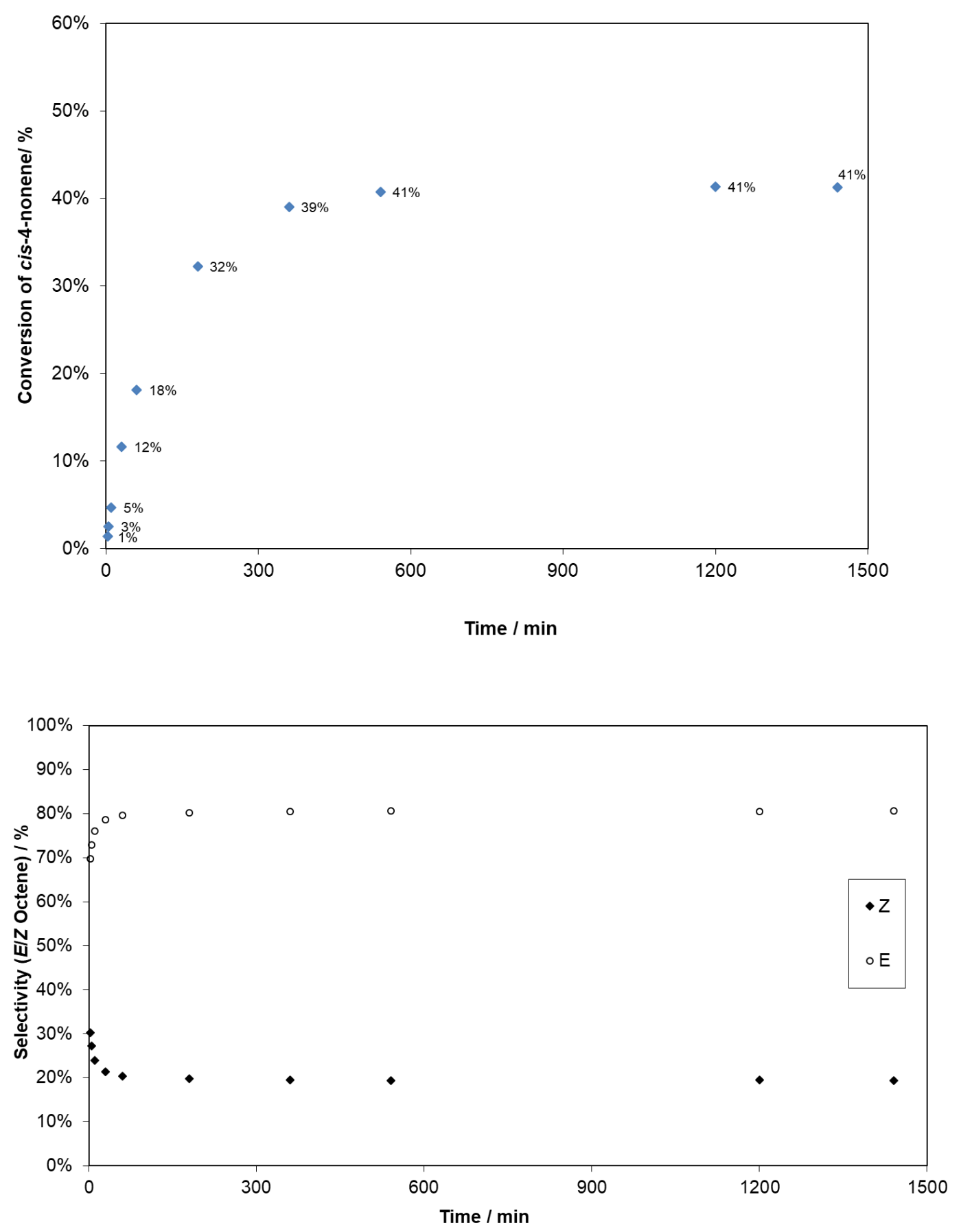


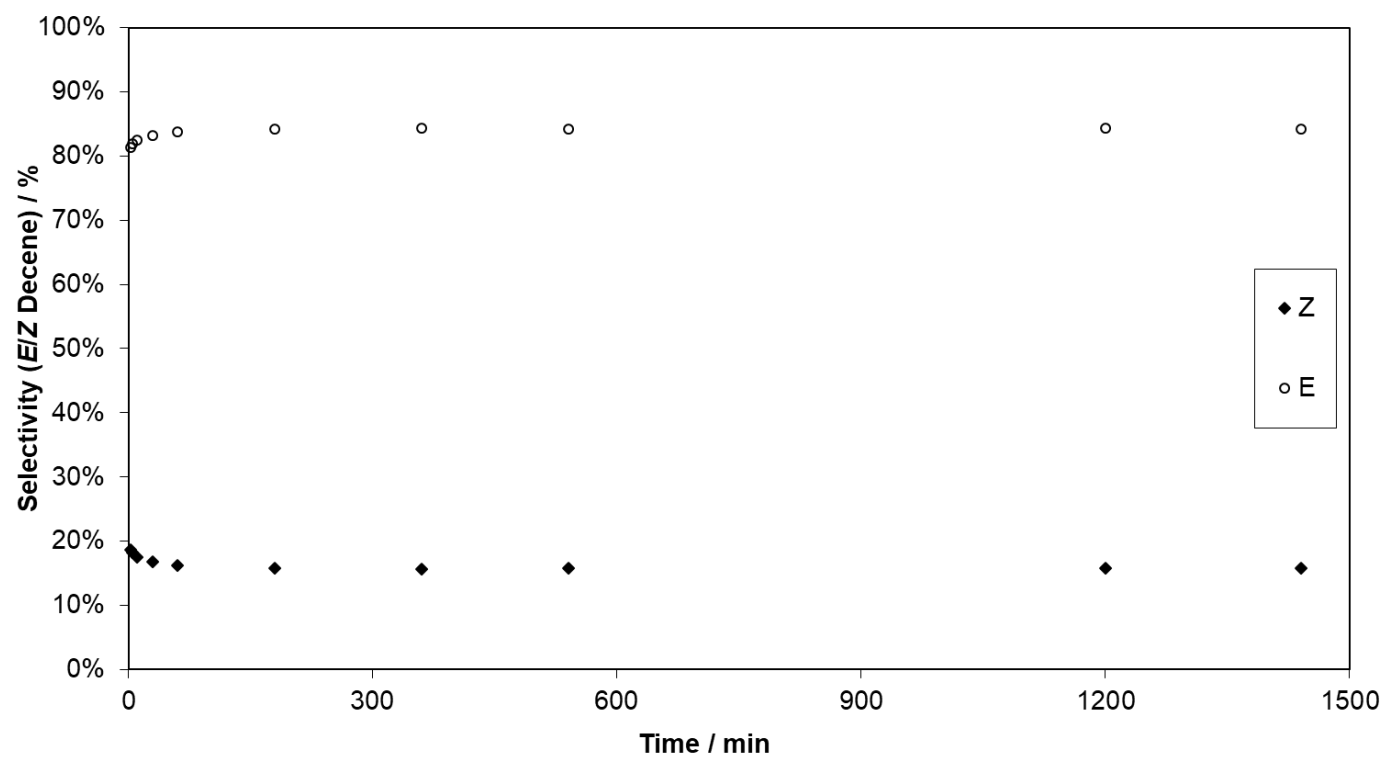

Figure S25. Conversion vs time and selectivity vs time plots for $\mathbf{1} @ \mathrm{SiO}_{2}(0.005 \mathrm{~mol} \%)$. 
Supporting Information
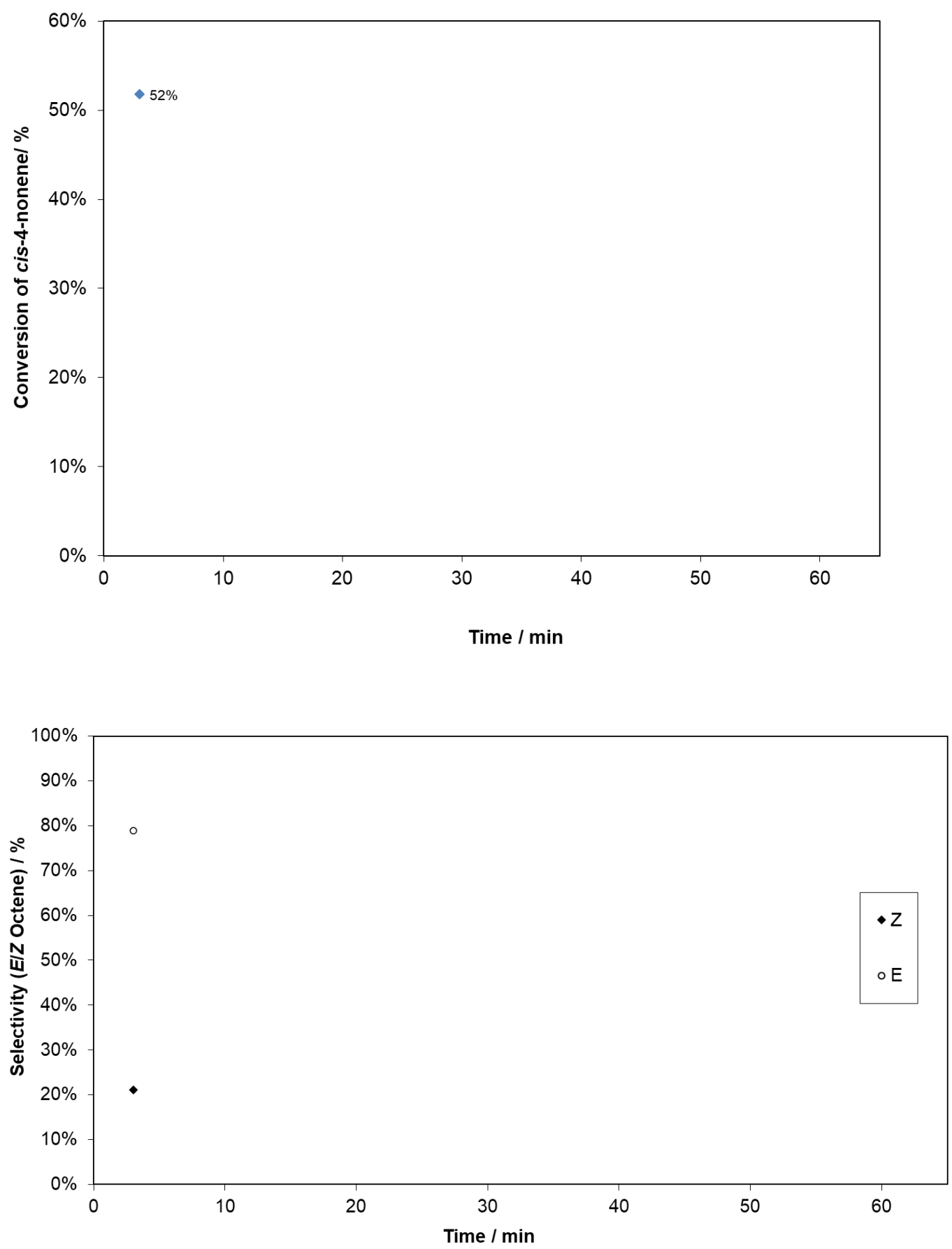


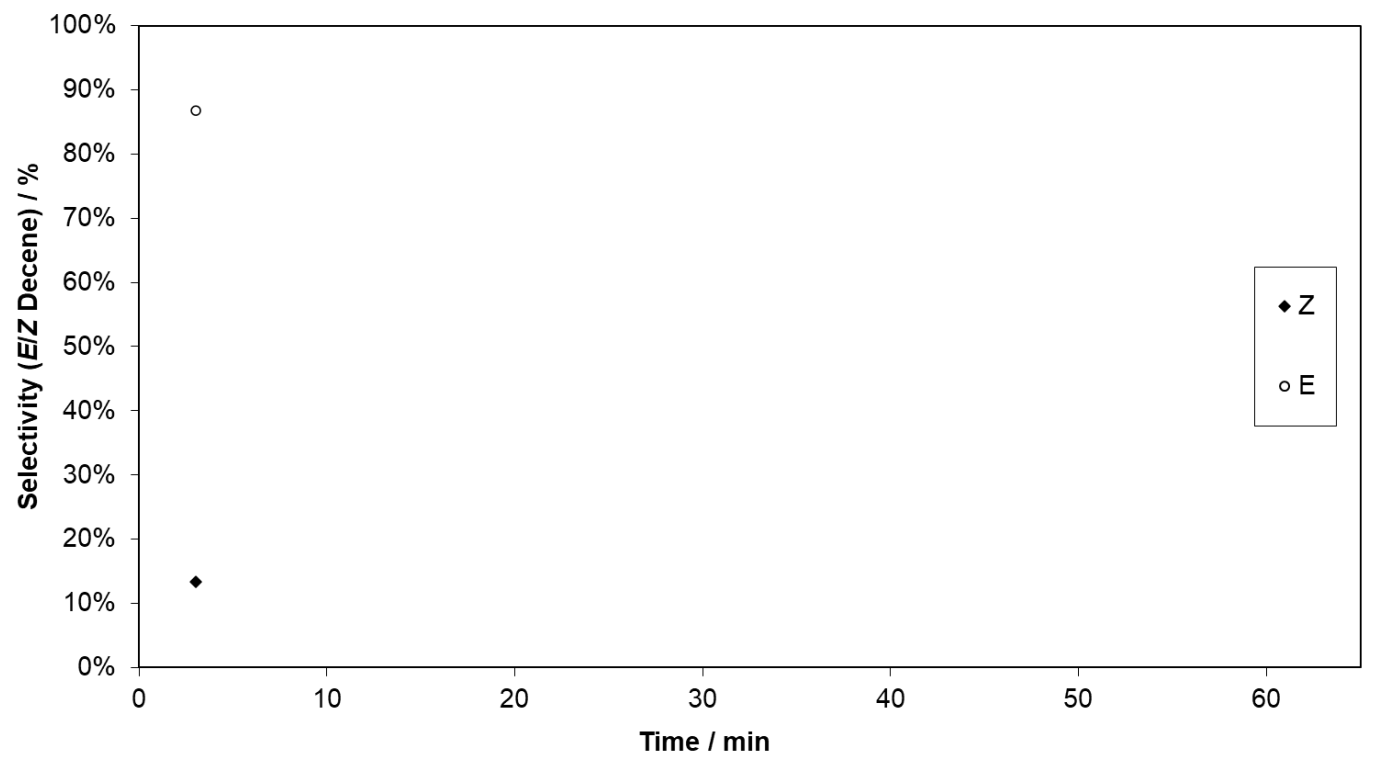

Figure S26. Conversion vs time and selectivity vs time plots for $2 @ \mathrm{SiO}_{2}(0.1 \mathrm{~mol} \%)$. Due to fast conversion, only one data point could be obtained. 

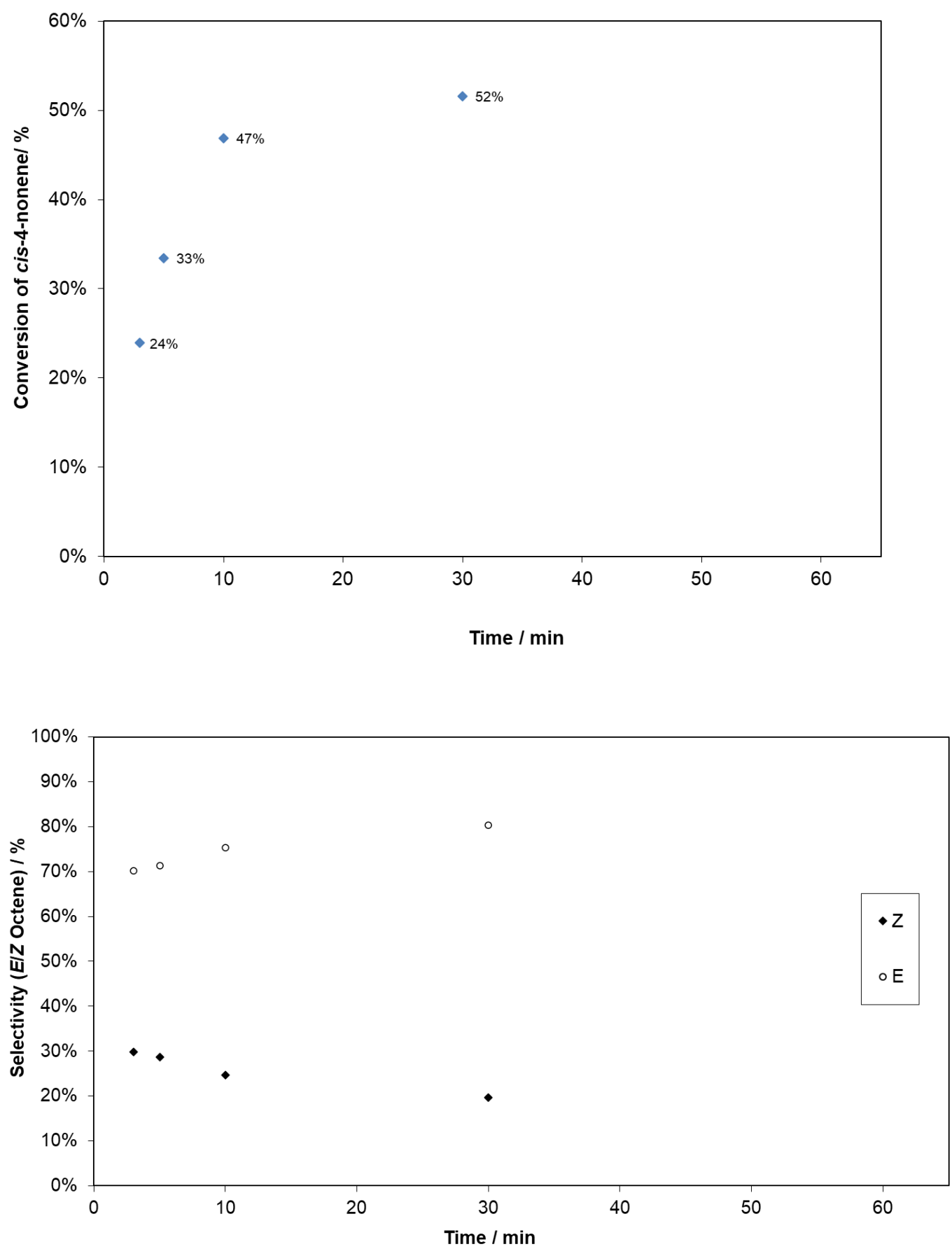


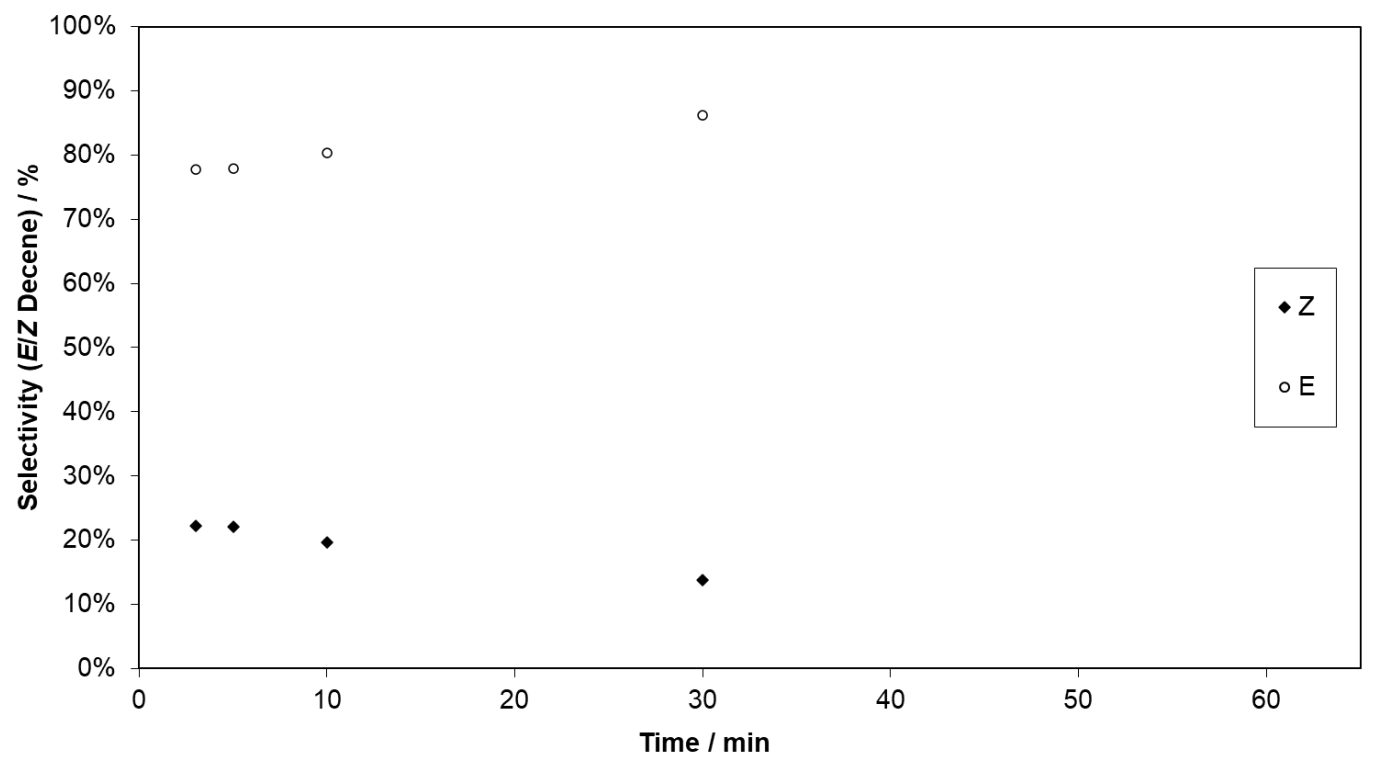

Figure S27. Conversion vs time and selectivity vs time plots for $\mathbf{2} @ \mathrm{SiO}_{2}(0.02 \mathrm{~mol} \%)$. 
Plots of Conversion vs Time and Selectivity vs Time for 1Nonene Experiments
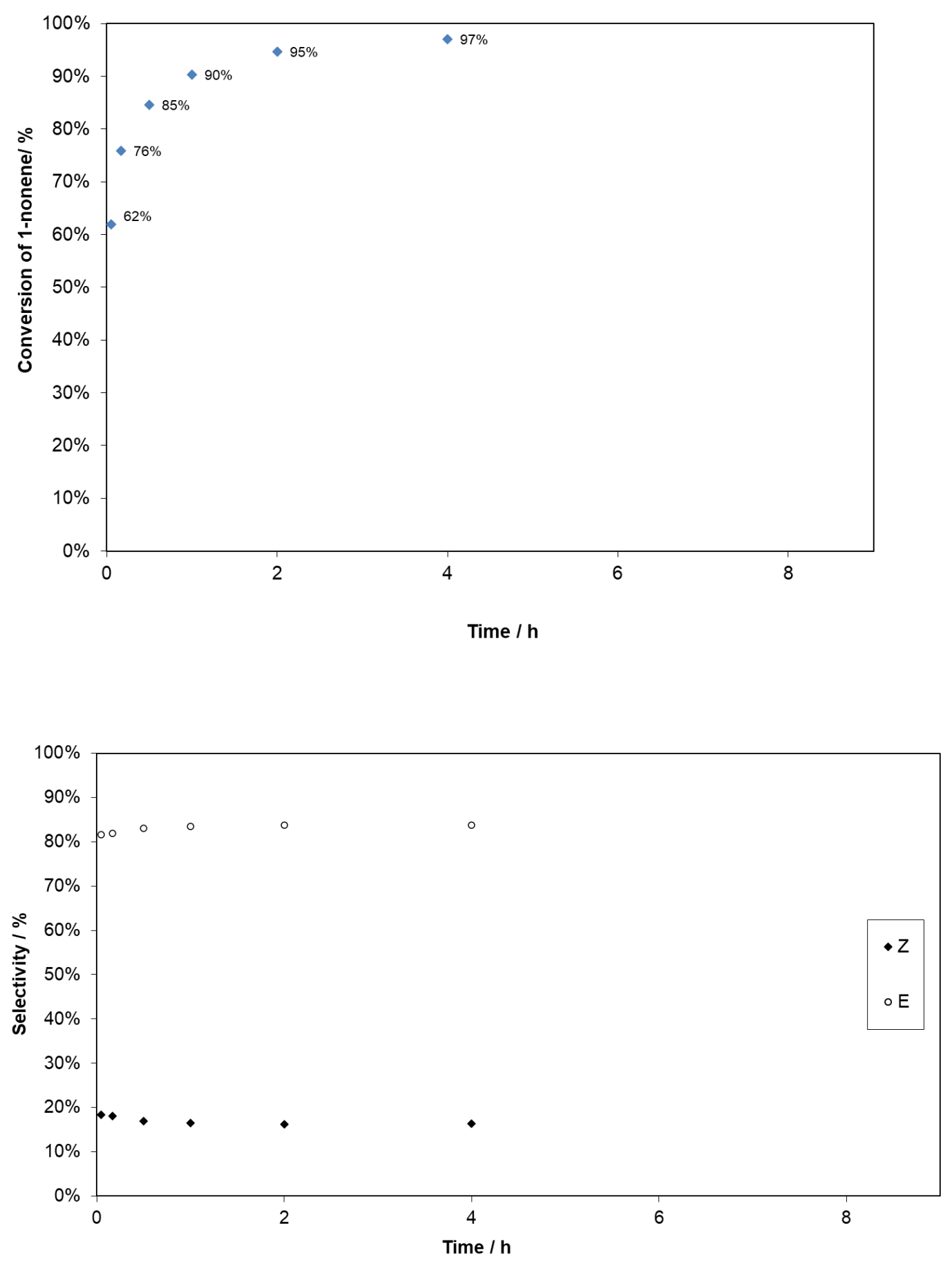

Figure S28. Conversion vs time and selectivity vs time plots for $\mathbf{1}(0.1 \mathrm{~mol} \%)$. 

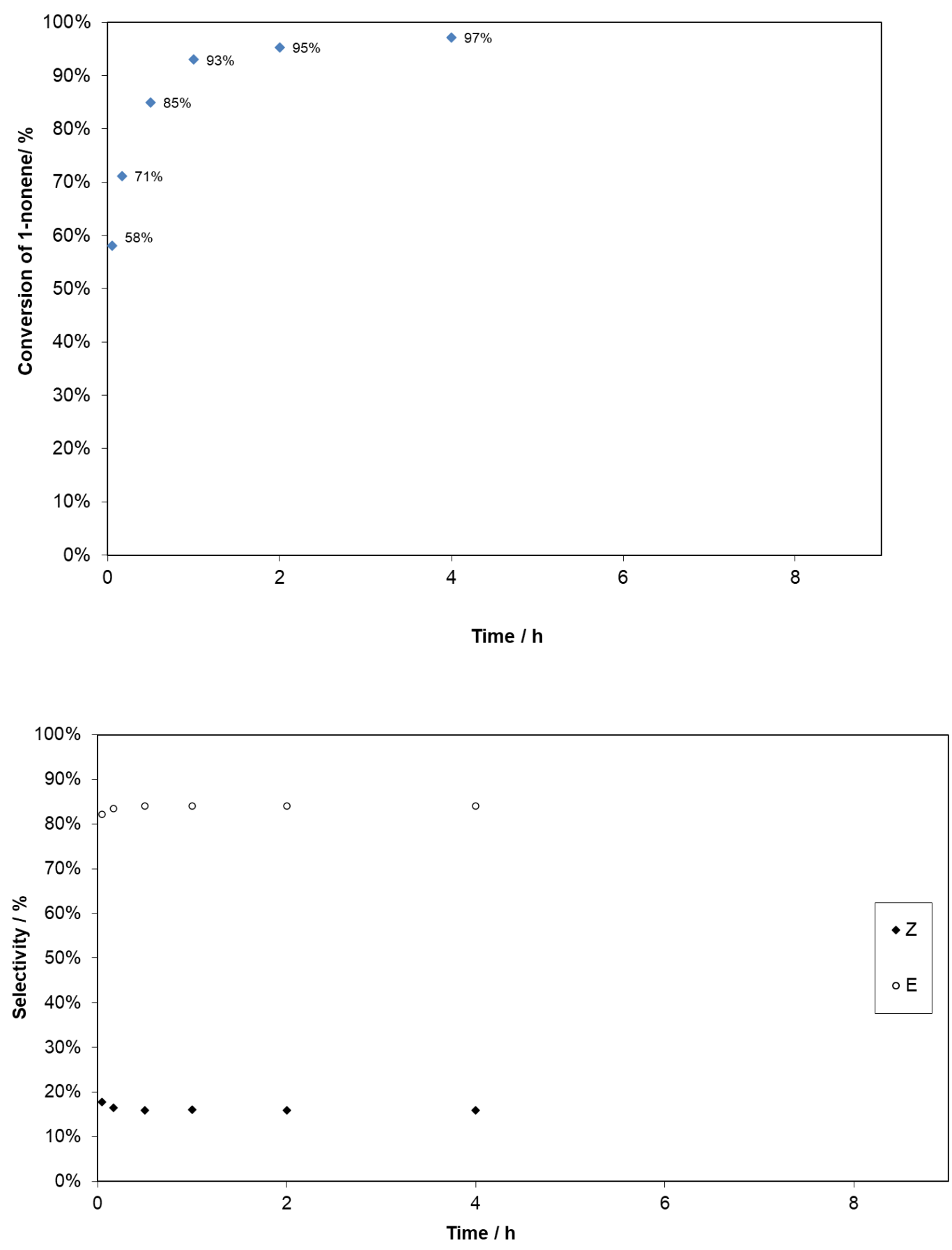

Figure S29. Conversion vs time and selectivity vs time plots for $1(0.02 \mathrm{~mol} \%)$. 

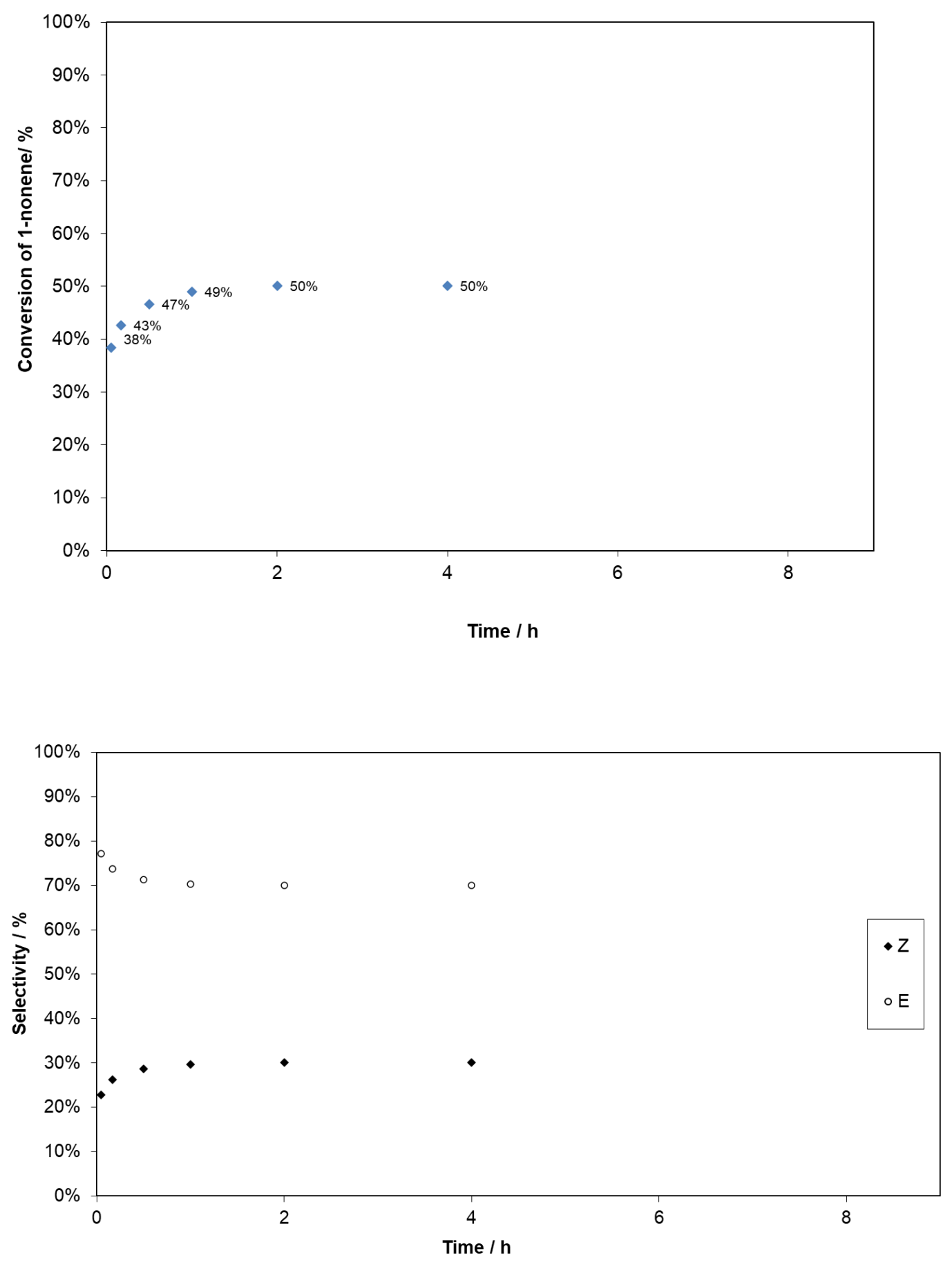

Figure S30. Conversion vs time and selectivity vs time plots for $\mathbf{1}(0.01 \mathrm{~mol} \%)$. 

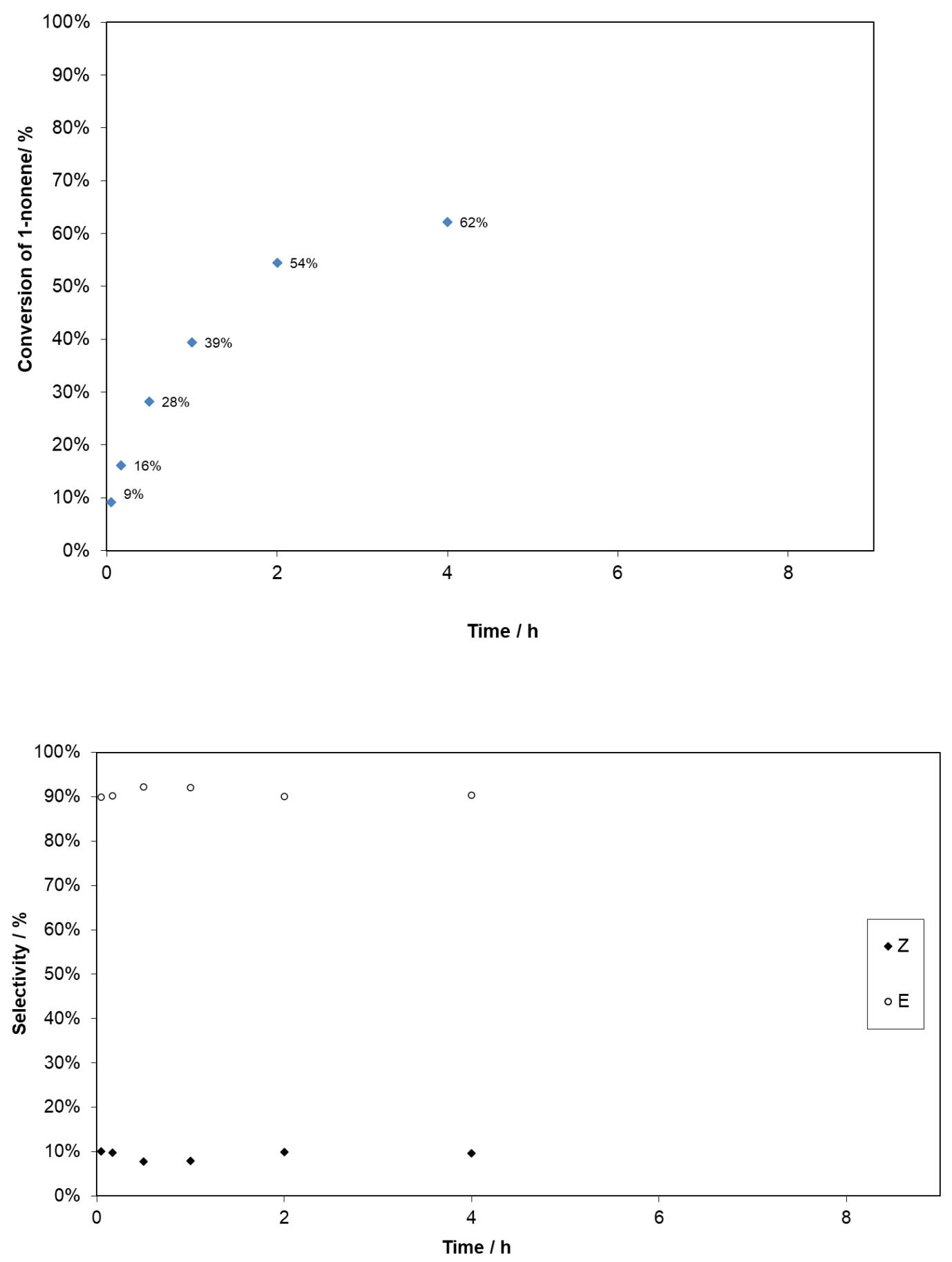

Figure S31. Conversion vs time and selectivity vs time plots for $\mathbf{2}(0.1 \mathrm{~mol} \%)$. 

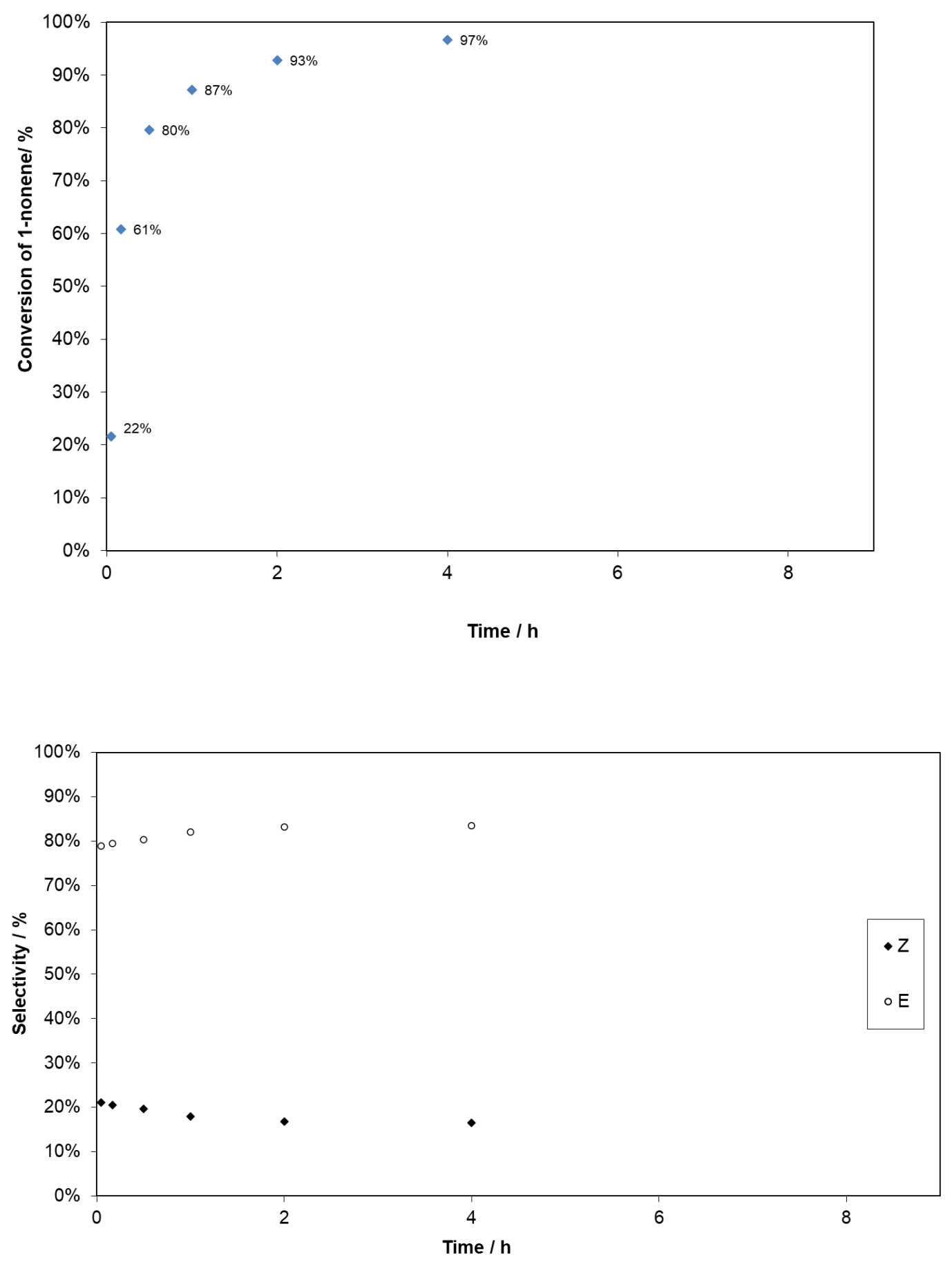

Figure S32. Conversion vs time and selectivity vs time plots for $1 @ \mathrm{SiO}_{2}(0.1 \mathrm{~mol} \%)$. 

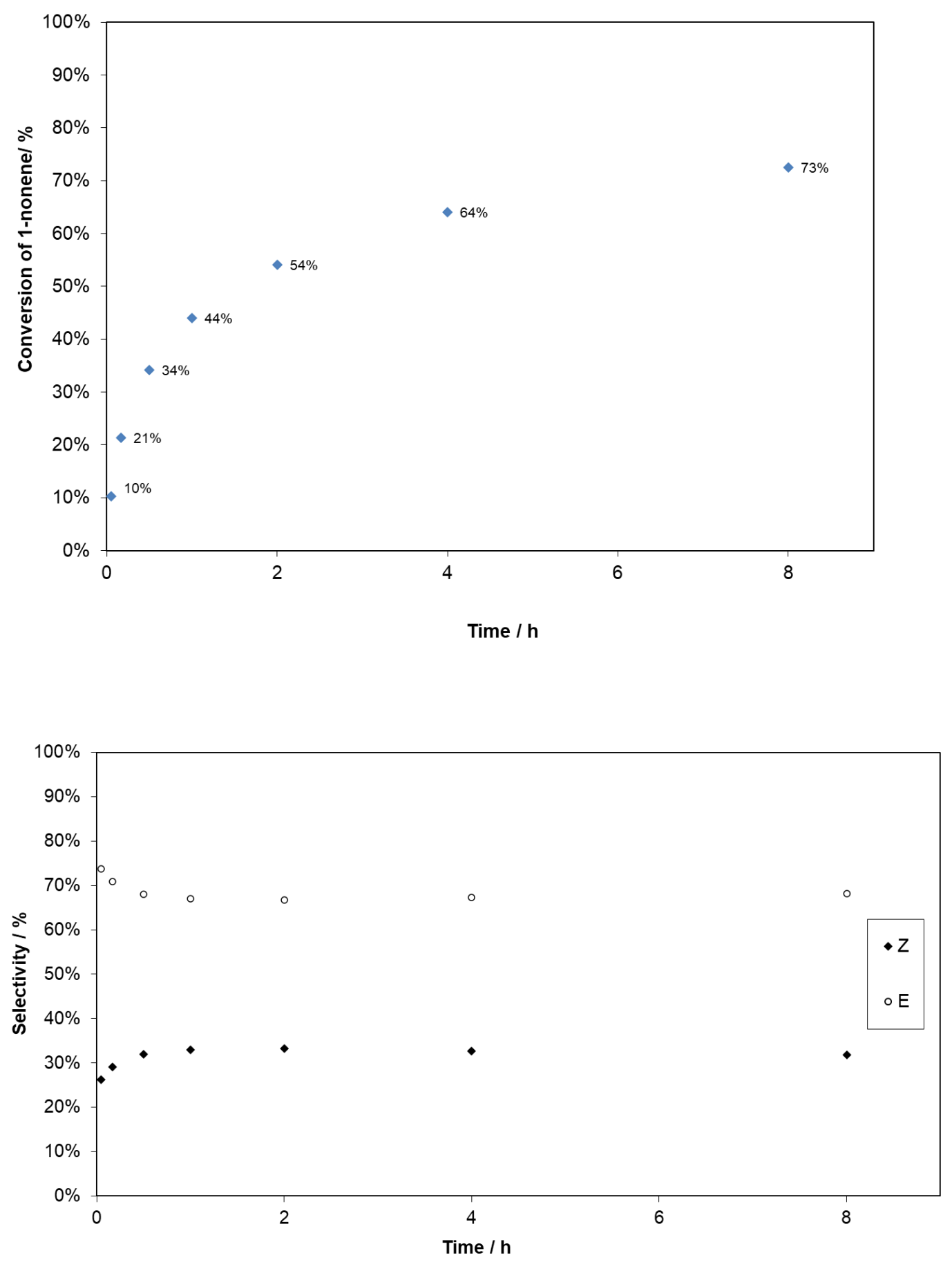

Figure S33. Conversion vs time and selectivity vs time plots for $1 @ \mathrm{SiO}_{2}(0.02 \mathrm{~mol} \%)$. 

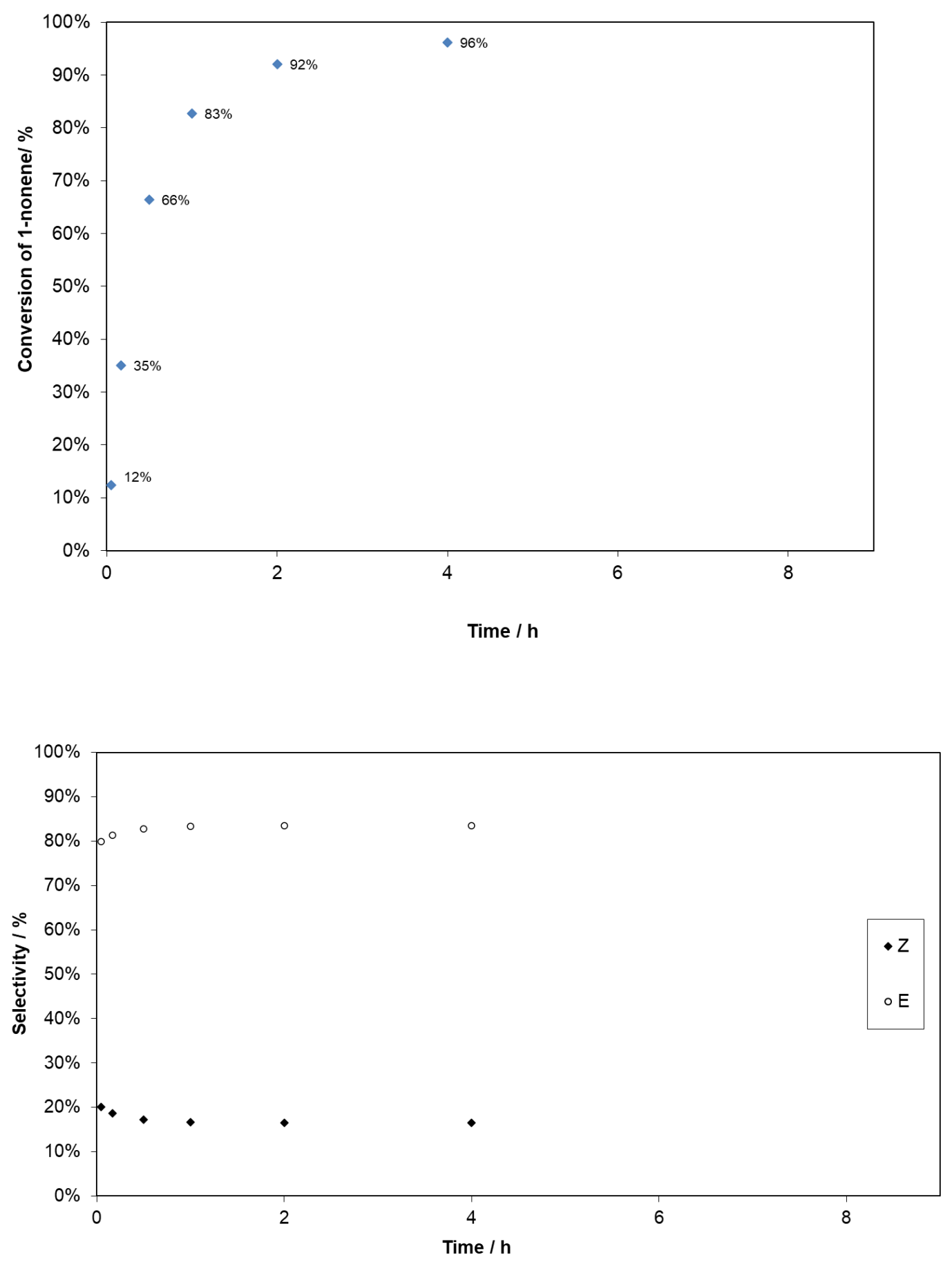

Figure S34. Conversion vs time and selectivity vs time plots for $2 @ \mathrm{SiO}_{2}(0.1 \mathrm{~mol} \%)$. 

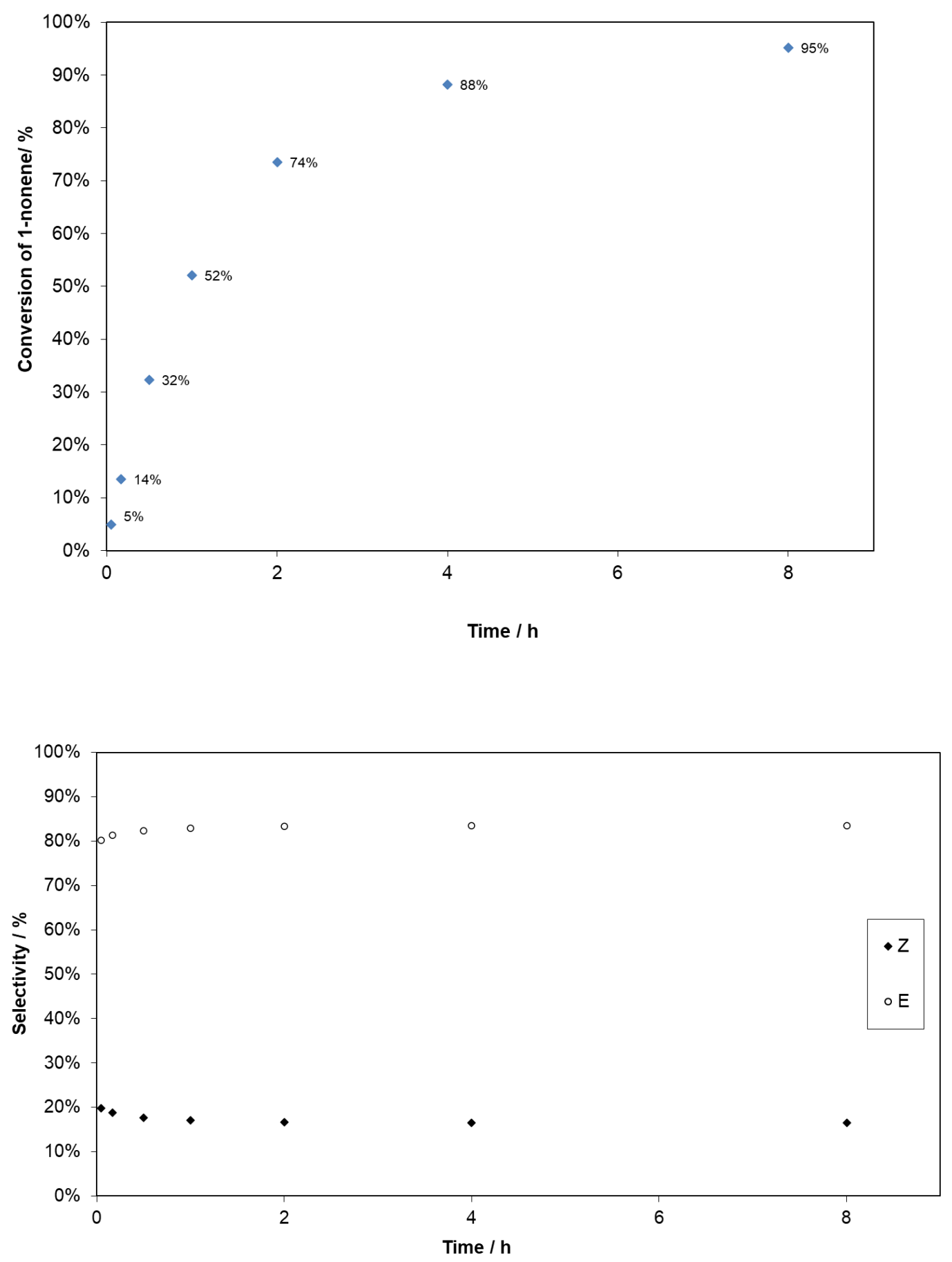

Figure S35. Conversion vs time and selectivity vs time plots for $\mathbf{2} @ \mathrm{SiO}_{2}(0.02 \mathrm{~mol} \%)$. 


\section{Crystallographic Data}

Table S3. Crystal data and structure refinement for 1 *

\begin{tabular}{|c|c|c|}
\hline Identification Code & \multicolumn{2}{|l|}{--} \\
\hline CCDC deposition number & \multicolumn{2}{|l|}{2053831} \\
\hline Empirical Formula & \multicolumn{2}{|c|}{$\mathrm{C}_{20} \mathrm{H}_{16} \mathrm{~F}_{18} \mathrm{MoO}_{5} * 1 / 2\left(\mathrm{C}_{5} \mathrm{H}_{12}\right)$} \\
\hline Formula Weight & \multicolumn{2}{|l|}{810.34} \\
\hline Temperature (K) & \multicolumn{2}{|l|}{100} \\
\hline Wavelength $(\AA)$ & \multicolumn{2}{|l|}{0.71073} \\
\hline Crystal System & \multicolumn{2}{|l|}{ tetragonal } \\
\hline Space Group & \multicolumn{2}{|l|}{$\mathrm{P}-42 \mathrm{c}$} \\
\hline \multirow[t]{3}{*}{ Unit Cell Dimensions } & $\mathrm{a}=23.2610(8) \AA$ & $\alpha=90^{\circ}$ \\
\hline & $\mathrm{b}=23.2610(8) \AA$ & $\beta=90^{\circ}$ \\
\hline & $c=10.6810(5) \AA$ & $\gamma=90^{\circ}$ \\
\hline Volume $\left(\AA^{3}\right)$ & \multicolumn{2}{|l|}{$5779.2(4)$} \\
\hline $\mathrm{Z}$ & \multicolumn{2}{|l|}{8} \\
\hline Calculated Density $\left(\mathrm{mg} / \mathrm{m}^{3}\right)$ & \multicolumn{2}{|l|}{1.863} \\
\hline Absorption Coefficient $\left(\mathrm{mm}^{-1}\right)$ & \multicolumn{2}{|l|}{0.604} \\
\hline $\mathrm{F}(000)$ & \multicolumn{2}{|l|}{3208} \\
\hline Crystal size $\left(\mathrm{mm}^{3}\right)$ & \multicolumn{2}{|c|}{$0.210 \times 0.190 \times 0.980$} \\
\hline Theta range for data collection $\left(^{\circ}\right)$ & \multicolumn{2}{|l|}{2.48 to 26.63} \\
\hline Index ranges & \multicolumn{2}{|c|}{$-29 \leq \mathrm{h} \leq 29,-29 \leq \mathrm{k} \leq 29,-13 \leq 1 \leq 13$} \\
\hline Reflections collected & \multicolumn{2}{|c|}{141086} \\
\hline Independent reflections & \multicolumn{2}{|l|}{6148} \\
\hline Completeness to theta $=50.5^{\circ}$ & \multicolumn{2}{|l|}{$99.9 \%$} \\
\hline Absorption Correction & \multicolumn{2}{|c|}{ Semi-empirical from equivalents } \\
\hline Max. and min. transmission & \multicolumn{2}{|c|}{0.886 and 0.865} \\
\hline Refinement method & \multicolumn{2}{|c|}{ Full-matrix least squares on $\mathrm{F}^{2}$} \\
\hline Data / restraints / parameters & \multicolumn{2}{|l|}{$6148 / 6 / 450$} \\
\hline Goodness-of-fit on $\mathrm{F}^{2}$ & \multicolumn{2}{|l|}{1.098} \\
\hline Final $\mathrm{R}$ indices $[\mathrm{I}>2 \sigma(\mathrm{I})]$ & \multicolumn{2}{|c|}{$\mathrm{R} 1=0.0209, \mathrm{wR} 2=0.0464$} \\
\hline $\mathrm{R}$ indices (all data) & \multicolumn{2}{|c|}{$\mathrm{R} 1=0.0245, \mathrm{wR} 2=0.0483$} \\
\hline Largest diff. peak and hole $\left(\mathrm{e} \bullet \AA^{-3}\right)$ & \multicolumn{2}{|l|}{0.210 and -0.328} \\
\hline
\end{tabular}


Table S4. Bond lengths for 1 *.

\begin{tabular}{|c|c|c|c|c|c|}
\hline Atom & Atom & Length $/ \AA$ & Atom & Atom & Length/Å \\
\hline Mo1 & $\mathrm{O} 1$ & $1.6745(19)$ & $\mathrm{C} 1$ & $\mathrm{C} 4$ & $1.562(4)$ \\
\hline Mo1 & $\mathrm{O} 2$ & $1.999(2)$ & $\mathrm{C} 5$ & C6 & $1.556(4)$ \\
\hline Mo1 & $\mathrm{O} 3$ & $1.990(2)$ & $\mathrm{C} 5$ & $\mathrm{C} 7$ & $1.565(4)$ \\
\hline Mo1 & $\mathrm{O} 4$ & $2.203(2)$ & $\mathrm{C} 5$ & $\mathrm{C} 8$ & $1.556(4)$ \\
\hline Mo1 & $\mathrm{C} 13$ & $1.909(3)$ & C9 & H9A & 0.99 \\
\hline $\mathrm{F} 1$ & $\mathrm{C} 2$ & $1.325(4)$ & C9 & H9B & 0.99 \\
\hline $\mathrm{F} 2$ & $\mathrm{C} 2$ & $1.338(4)$ & C9 & $\mathrm{C} 10$ & $1.523(5)$ \\
\hline F3 & $\mathrm{C} 2$ & $1.339(3)$ & $\mathrm{C} 10$ & $\mathrm{H} 10 \mathrm{~A}$ & 0.99 \\
\hline F4 & $\mathrm{C} 3$ & $1.344(4)$ & $\mathrm{C} 10$ & H10B & 0.99 \\
\hline F5 & $\mathrm{C} 3$ & $1.329(4)$ & $\mathrm{C} 10$ & $\mathrm{C} 11$ & $1.517(5)$ \\
\hline F6 & $\mathrm{C} 3$ & $1.339(4)$ & $\mathrm{C} 11$ & H11A & 0.99 \\
\hline F7 & $\mathrm{C} 4$ & $1.334(4)$ & $\mathrm{C} 11$ & H11B & 0.99 \\
\hline F8 & $\mathrm{C} 4$ & $1.347(4)$ & $\mathrm{C} 11$ & $\mathrm{C} 12$ & $1.509(5)$ \\
\hline F9 & $\mathrm{C} 4$ & $1.322(4)$ & $\mathrm{C} 12$ & $\mathrm{H} 12 \mathrm{~A}$ & 0.99 \\
\hline F10 & C6 & $1.330(4)$ & $\mathrm{C} 12$ & H12B & 0.99 \\
\hline F11 & $\mathrm{C} 6$ & $1.338(4)$ & $\mathrm{C} 13$ & $\mathrm{C} 14$ & $1.441(4)$ \\
\hline F12 & C6 & $1.342(4)$ & $\mathrm{C} 13$ & H13 & $0.84(4)$ \\
\hline F13 & $\mathrm{C} 8$ & $1.335(4)$ & $\mathrm{C} 14$ & $\mathrm{C} 15$ & $1.403(4)$ \\
\hline F14 & $\mathrm{C} 8$ & $1.333(4)$ & $\mathrm{C} 14$ & $\mathrm{C} 16$ & $1.403(4)$ \\
\hline F15 & $\mathrm{C} 8$ & $1.340(4)$ & $\mathrm{C} 15$ & H15 & 0.95 \\
\hline F16 & $\mathrm{C} 7$ & $1.329(4)$ & $\mathrm{C} 15$ & $\mathrm{C} 17$ & $1.385(4)$ \\
\hline F17 & $\mathrm{C} 7$ & $1.340(4)$ & $\mathrm{C} 16$ & H16 & 0.95 \\
\hline F18 & $\mathrm{C} 7$ & $1.329(4)$ & $\mathrm{C} 16$ & $\mathrm{C} 18$ & $1.378(4)$ \\
\hline $\mathrm{O} 2$ & $\mathrm{C} 5$ & $1.363(4)$ & $\mathrm{C} 17$ & H17 & 0.95 \\
\hline $\mathrm{O} 3$ & $\mathrm{C} 1$ & $1.367(4)$ & $\mathrm{C} 17$ & $\mathrm{C} 19$ & $1.393(4)$ \\
\hline $\mathrm{O} 4$ & C9 & $1.456(4)$ & $\mathrm{C} 18$ & H18 & 0.95 \\
\hline $\mathrm{O} 4$ & $\mathrm{C} 12$ & $1.457(4)$ & $\mathrm{C} 18$ & $\mathrm{C} 19$ & $1.401(4)$ \\
\hline O5 & $\mathrm{C} 19$ & $1.355(4)$ & $\mathrm{C} 20$ & $\mathrm{H} 20 \mathrm{~A}$ & 0.98 \\
\hline O5 & $\mathrm{C} 20$ & $1.433(4)$ & $\mathrm{C} 20$ & H20B & 0.98 \\
\hline $\mathrm{C} 1$ & $\mathrm{C} 2$ & $1.557(4)$ & $\mathrm{C} 20$ & $\mathrm{H} 20 \mathrm{C}$ & 0.98 \\
\hline $\mathrm{C} 1$ & $\mathrm{C} 3$ & $1.552(4)$ & & & \\
\hline
\end{tabular}


Table S5. Bond angles for 1*.

\begin{tabular}{|c|c|c|c|c|c|c|c|}
\hline Atom & Atom & Atom & Angle $/^{\circ}$ & Atom & Atom & Atom & Angle $/^{\circ}$ \\
\hline$\overline{\mathrm{O} 1}$ & Mo1 & $\mathrm{O} 2$ & $97.79(9)$ & O4 & C9 & H9B & 110.6 \\
\hline $\mathrm{O} 1$ & Mo1 & $\mathrm{O} 3$ & $145.52(9)$ & $\mathrm{O} 4$ & $\mathrm{C} 9$ & $\mathrm{C} 10$ & $105.5(3)$ \\
\hline $\mathrm{O} 1$ & Mo1 & $\mathrm{O} 4$ & $85.75(8)$ & H9A & $\mathrm{C} 9$ & H9B & 108.8 \\
\hline $\mathrm{O} 1$ & Mo1 & $\mathrm{C} 13$ & $102.73(12)$ & $\mathrm{C} 10$ & C9 & H9A & 110.6 \\
\hline $\mathrm{O} 2$ & Mo1 & $\mathrm{O} 4$ & $162.75(8)$ & $\mathrm{C} 10$ & C9 & H9B & 110.6 \\
\hline $\mathrm{O} 3$ & Mo1 & $\mathrm{O} 2$ & $87.78(8)$ & C9 & $\mathrm{C} 10$ & H10A & 110.8 \\
\hline $\mathrm{O} 3$ & Mo1 & $\mathrm{O} 4$ & $80.05(8)$ & C9 & $\mathrm{C} 10$ & H10B & 110.8 \\
\hline $\mathrm{C} 13$ & Mo1 & $\mathrm{O} 2$ & $103.28(11)$ & $\mathrm{H} 10 \mathrm{~A}$ & $\mathrm{C} 10$ & H10B & 108.8 \\
\hline $\mathrm{C} 13$ & Mo1 & $\mathrm{O} 3$ & $109.05(11)$ & $\mathrm{C} 11$ & $\mathrm{C} 10$ & C9 & $105.0(3)$ \\
\hline $\mathrm{C} 13$ & Mo1 & $\mathrm{O} 4$ & $92.30(10)$ & $\mathrm{C} 11$ & $\mathrm{C} 10$ & H10A & 110.8 \\
\hline C5 & $\mathrm{O} 2$ & Mo1 & $142.00(19)$ & $\mathrm{C} 11$ & $\mathrm{C} 10$ & H10B & 110.8 \\
\hline $\mathrm{C} 1$ & $\mathrm{O} 3$ & Mo1 & $142.29(18)$ & $\mathrm{C} 10$ & $\mathrm{C} 11$ & H11A & 110.7 \\
\hline C9 & $\mathrm{O} 4$ & Mo1 & $122.86(19)$ & $\mathrm{C} 10$ & $\mathrm{C} 11$ & H11B & 110.7 \\
\hline C9 & $\mathrm{O} 4$ & $\mathrm{C} 12$ & $105.7(2)$ & $\mathrm{H} 11 \mathrm{~A}$ & C11 & H11B & 108.8 \\
\hline $\mathrm{C} 12$ & $\mathrm{O} 4$ & Mo1 & $122.75(18)$ & $\mathrm{C} 12$ & C11 & $\mathrm{C} 10$ & $105.2(3)$ \\
\hline $\mathrm{C} 19$ & O5 & $\mathrm{C} 20$ & $117.2(2)$ & $\mathrm{C} 12$ & C11 & H11A & 110.7 \\
\hline $\mathrm{O} 3$ & $\mathrm{C} 1$ & $\mathrm{C} 2$ & $109.5(3)$ & $\mathrm{C} 12$ & C11 & H11B & 110.7 \\
\hline $\mathrm{O} 3$ & $\mathrm{C} 1$ & $\mathrm{C} 3$ & $109.0(2)$ & O4 & $\mathrm{C} 12$ & $\mathrm{C} 11$ & $103.4(3)$ \\
\hline $\mathrm{O} 3$ & $\mathrm{C} 1$ & $\mathrm{C} 4$ & $110.3(2)$ & $\mathrm{O} 4$ & $\mathrm{C} 12$ & $\mathrm{H} 12 \mathrm{~A}$ & 111.1 \\
\hline $\mathrm{C} 2$ & $\mathrm{C} 1$ & $\mathrm{C} 4$ & $110.1(2)$ & $\mathrm{O} 4$ & $\mathrm{C} 12$ & H12B & 111.1 \\
\hline $\mathrm{C} 3$ & $\mathrm{C} 1$ & $\mathrm{C} 2$ & $108.7(2)$ & $\mathrm{C} 11$ & $\mathrm{C} 12$ & $\mathrm{H} 12 \mathrm{~A}$ & 111.1 \\
\hline $\mathrm{C} 3$ & $\mathrm{C} 1$ & $\mathrm{C} 4$ & 109.2(3) & $\mathrm{C} 11$ & $\mathrm{C} 12$ & H12B & 111.1 \\
\hline F1 & $\mathrm{C} 2$ & $\mathrm{~F} 2$ & $107.7(3)$ & $\mathrm{H} 12 \mathrm{~A}$ & $\mathrm{C} 12$ & H12B & 109 \\
\hline $\mathrm{F} 1$ & $\mathrm{C} 2$ & F3 & $107.9(2)$ & Mo1 & $\mathrm{C} 13$ & H13 & $99(3)$ \\
\hline $\mathrm{F} 1$ & $\mathrm{C} 2$ & $\mathrm{C} 1$ & $110.9(2)$ & $\mathrm{C} 14$ & $\mathrm{C} 13$ & Mo1 & $138.7(2)$ \\
\hline $\mathrm{F} 2$ & $\mathrm{C} 2$ & F3 & $107.5(2)$ & $\mathrm{C} 14$ & $\mathrm{C} 13$ & H13 & $122(3)$ \\
\hline $\mathrm{F} 2$ & $\mathrm{C} 2$ & $\mathrm{C} 1$ & $110.5(2)$ & $\mathrm{C} 15$ & $\mathrm{C} 14$ & $\mathrm{C} 13$ & $119.9(3)$ \\
\hline F3 & $\mathrm{C} 2$ & $\mathrm{C} 1$ & $112.3(3)$ & $\mathrm{C} 16$ & $\mathrm{C} 14$ & $\mathrm{C} 13$ & $122.4(3)$ \\
\hline F4 & $\mathrm{C} 3$ & $\mathrm{C} 1$ & $110.9(3)$ & $\mathrm{C} 16$ & $\mathrm{C} 14$ & $\mathrm{C} 15$ & $117.7(3)$ \\
\hline F5 & $\mathrm{C} 3$ & $\mathrm{~F} 4$ & $107.4(3)$ & $\mathrm{C} 14$ & $\mathrm{C} 15$ & H15 & 119.1 \\
\hline F5 & $\mathrm{C} 3$ & F6 & $108.2(3)$ & $\mathrm{C} 17$ & $\mathrm{C} 15$ & $\mathrm{C} 14$ & $121.7(3)$ \\
\hline F5 & $\mathrm{C} 3$ & $\mathrm{C} 1$ & 111.2(3) & $\mathrm{C} 17$ & $\mathrm{C} 15$ & H15 & 119.1 \\
\hline F6 & $\mathrm{C} 3$ & $\mathrm{~F} 4$ & $107.4(3)$ & $\mathrm{C} 14$ & $\mathrm{C} 16$ & H16 & 119.5 \\
\hline F6 & $\mathrm{C} 3$ & $\mathrm{C} 1$ & $111.5(3)$ & $\mathrm{C} 18$ & $\mathrm{C} 16$ & $\mathrm{C} 14$ & $121.0(3)$ \\
\hline F7 & $\mathrm{C} 4$ & F8 & $107.2(3)$ & $\mathrm{C} 18$ & $\mathrm{C} 16$ & H16 & 119.5 \\
\hline F7 & $\mathrm{C} 4$ & $\mathrm{C} 1$ & $112.7(3)$ & $\mathrm{C} 15$ & $\mathrm{C} 17$ & H17 & 120.2 \\
\hline F8 & $\mathrm{C} 4$ & $\mathrm{C} 1$ & 109.1(3) & $\mathrm{C} 15$ & $\mathrm{C} 17$ & C19 & $119.6(3)$ \\
\hline F9 & $\mathrm{C} 4$ & F7 & $108.6(3)$ & $\mathrm{C} 19$ & $\mathrm{C} 17$ & H17 & 120.2 \\
\hline F9 & $\mathrm{C} 4$ & F8 & $107.5(3)$ & $\mathrm{C} 16$ & C18 & H18 & 119.8 \\
\hline F9 & $\mathrm{C} 4$ & $\mathrm{C} 1$ & $111.6(3)$ & $\mathrm{C} 16$ & $\mathrm{C} 18$ & $\mathrm{C} 19$ & $120.4(3)$ \\
\hline $\mathrm{O} 2$ & $\mathrm{C} 5$ & $\mathrm{C} 6$ & $111.6(2)$ & $\mathrm{C} 19$ & $\mathrm{C} 18$ & H18 & 119.8 \\
\hline $\mathrm{O} 2$ & $\mathrm{C} 5$ & $\mathrm{C} 7$ & $106.7(2)$ & O5 & C19 & $\mathrm{C} 17$ & $124.3(3)$ \\
\hline $\mathrm{O} 2$ & $\mathrm{C} 5$ & $\mathrm{C} 8$ & $111.0(2)$ & $\mathrm{O} 5$ & C19 & $\mathrm{C} 18$ & $116.2(2)$ \\
\hline C6 & $\mathrm{C} 5$ & $\mathrm{C} 7$ & $108.9(2)$ & $\mathrm{C} 17$ & $\mathrm{C} 19$ & $\mathrm{C} 18$ & $119.5(3)$ \\
\hline $\mathrm{C} 8$ & $\mathrm{C} 5$ & C6 & $109.6(2)$ & O5 & $\mathrm{C} 20$ & $\mathrm{H} 20 \mathrm{~A}$ & 109.5 \\
\hline $\mathrm{C} 8$ & $\mathrm{C} 5$ & $\mathrm{C} 7$ & $108.8(2)$ & $\mathrm{O} 5$ & $\mathrm{C} 20$ & H20B & 109.5 \\
\hline F10 & C6 & F11 & $107.4(3)$ & $\mathrm{O} 5$ & $\mathrm{C} 20$ & $\mathrm{H} 20 \mathrm{C}$ & 109.5 \\
\hline F10 & C6 & F12 & $107.8(3)$ & $\mathrm{H} 20 \mathrm{~A}$ & $\mathrm{C} 20$ & H20B & 109.5 \\
\hline F10 & C6 & $\mathrm{C} 5$ & $110.8(3)$ & $\mathrm{H} 20 \mathrm{~A}$ & $\mathrm{C} 20$ & $\mathrm{H} 20 \mathrm{C}$ & 109.5 \\
\hline F11 & $\mathrm{C} 6$ & F12 & 107.1(3) & H20B & $\mathrm{C} 20$ & $\mathrm{H} 20 \mathrm{C}$ & 109.5 \\
\hline
\end{tabular}




\begin{tabular}{llllllll} 
F11 & C6 & C5 & $111.2(3)$ & F13 & C8 & F15 & $107.6(2)$ \\
F12 & C6 & C5 & $112.3(3)$ & F13 & C8 & C5 & $110.7(3)$ \\
F16 & C7 & F17 & $107.5(3)$ & F14 & C8 & F13 & $107.5(2)$ \\
F16 & C7 & C5 & $110.8(3)$ & F14 & C8 & F15 & $107.5(3)$ \\
F17 & C7 & C5 & $112.5(3)$ & F14 & C8 & C5 & $111.0(2)$ \\
F18 & C7 & F16 & $107.7(3)$ & F15 & C8 & C5 & $112.4(2)$ \\
F18 & C7 & F17 & $107.3(3)$ & O4 & C9 & H9A & 110.6 \\
F18 & C7 & C5 & $110.7(3)$ & & & & \\
\hline
\end{tabular}

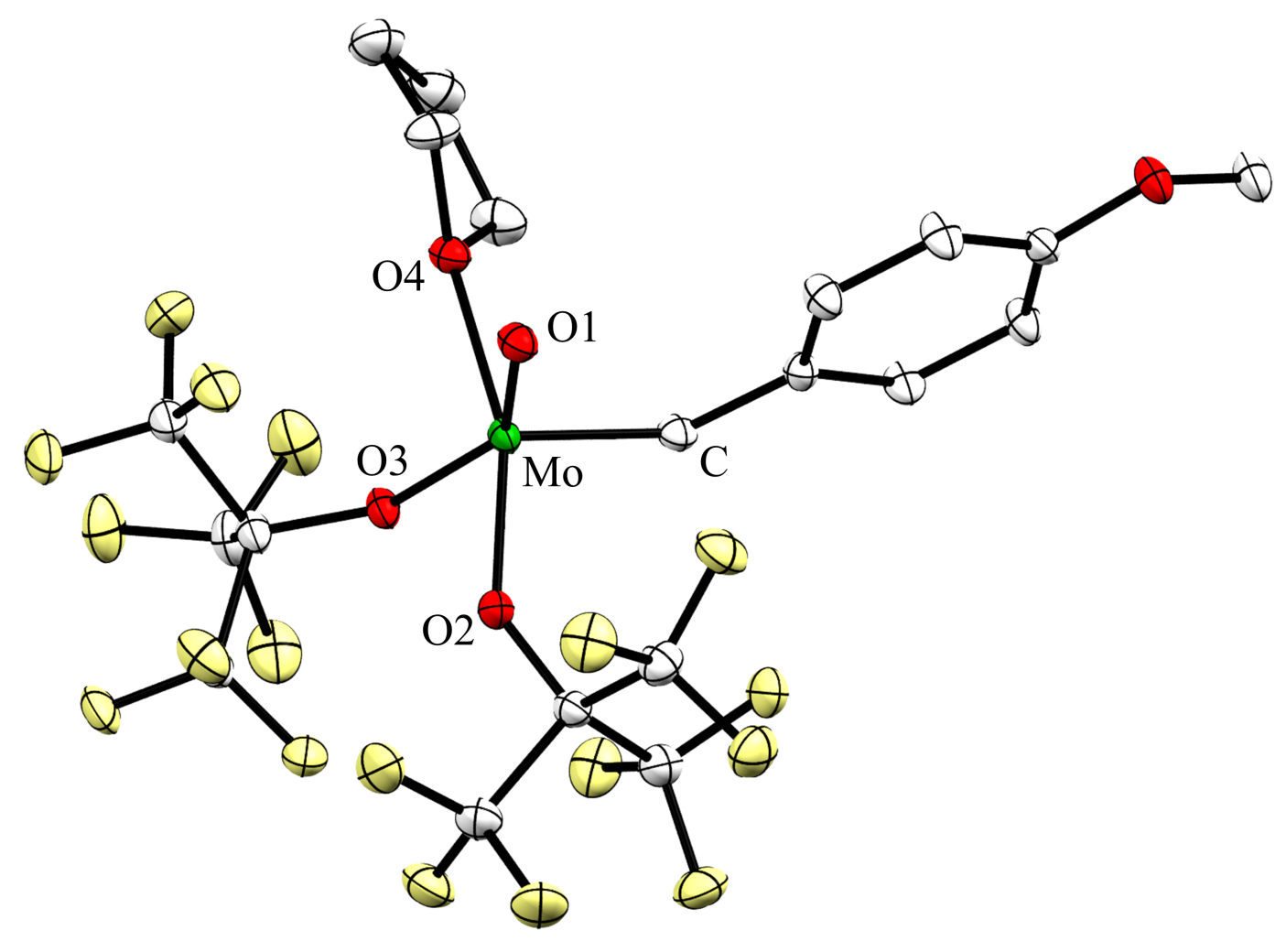

Figure S36. X-ray crystal structure of $1 *$. Co-crystallized $n$-pentane and hydrogen atoms have been omitted for clarity. Thermal ellipsoids are set to $50 \%$ probability.

Selected bond lengths $[\AA]$ and bond angles $[\mathrm{deg}]$ for $1 *$ : Mo-O1 1.674, Mo-O2 2.000, Mo-O3 1.990, Mo-O4 2.203, Mo-C 1.909, O1-Mo-O2 97.79, O1-Mo-O3 145.52, O1-Mo-O4 85.76, O1-Mo-C 102.73, O2-Mo-O3 87.78, O2-Mo-O4 162.75, O2-Mo-C 103.27, O3-Mo-O4 80.06, O3-Mo-C 109.05, O4-Mo-C 92.30. 


\section{References}

[1] B. M. Fung, A. K. Khitrin, K. Ermolaev, An Improved Broadband Decoupling Sequence for Liquid Crystals and Solids. J. Magn. Reson. 2000, 142, 97-101.

[2] C. Copéret, A. Comas-Vives, M. P. Conley, D. P. Estes, A. Fedorov, V. Mougel, H. Nagae, F. Núñez-Zarur, P. A. Zhizhko, Surface Organometallic and Coordination Chemistry toward Single-Site Heterogeneous Catalysts: Strategies, Methods, Structures, and Activities. Chem. Rev. 2016, 116, 323-421.

[3] F. Zhai, R. R. Schrock, A. H. Hoveyda, P. Müller, Syntheses of "Phosphine-Free" Molybdenum Oxo Alkylidene Complexes through Addition of Water to Alkylidyne Complexes. Organometallics 2020, 39, 2486-2492.

[4] a) S. Grimme, C. Bannwarth, P. Shushkov, A Robust and Accurate Tight-Binding Quantum Chemical Method for Structures, Vibrational Frequencies, and Noncovalent Interactions of Large Molecular Systems Parametrized for All spd-Block Elements $(Z=1-86)$. JCTC 2017, 13 (5), 1989-2009. b) S. Spicher, S. Grimme, Robust Atomistic Modeling of Materials, Organometallic, and Biochemical Systems. Angew. Chem., Int. Ed. 2020, 59, 15665 15673. c) P. Pracht, F. Bohle, S. Grimme, Automated exploration of the low-energy chemical space with fast quantum chemical methods. Phys. Chem. Chem. Phys. 2020, 22, 7169-7192.

[5] J. P. Perdew, K. Burke, M. Ernzerhof, Generalized Gradient Approximation Made Simple. Phys. Rev. Lett. 1996, 77, 3865-3868.

[6] S. Grimme, S. Ehrlich, L. Goerigk, Effect of the damping function in dispersion corrected density functional theory. J. Comput. Chem. 2011, 32, 1456-1465.

[7] T. H. Dunning Jr., P. J. Hay, Modern Theoretical Chemistry, Ed. H. F. Schaefer III, Vol. 3 (Plenum, New York, 1977), 1-28.

[8] A. Schaefer, C. Huber, R. Ahlrichs, Fully optimized contracted Gaussian basis sets of triple zeta valence quality for atoms Li to Kr. J. Chem. Phys. 1994, 100, 5829-5835.

[9] C. Adamo, Toward reliable density functional methods without adjustable parameters: The PBE0 model. J. Chem. Phys. 1999, 110, 6158-6170. 